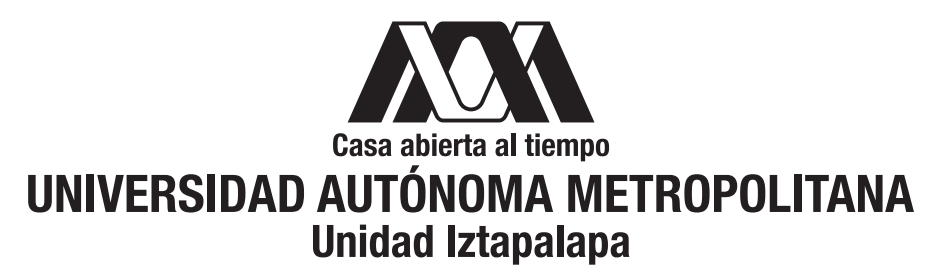

División de Ciencias Básica e Ingeniería Universidad Autónoma Metropolitana Unidad Iztapalapa

\title{
Efecto del Intercambio Exacto sobre el Band Gap en Óxidos Metálicos
}

\author{
Tesis que presenta \\ Marcos Rivera Almazo \\ para obtener el grado de \\ Maestro en Ciencias (Química) \\ Asesor \\ Jorge Garza Olguín
}

Jurado:

Presidente: Dr. Marcelo Enrique Galván Espinosa

Secretario: Dr. Jorge Garza Olguín

Vocal: Dr. José Eduardo Barrios Vargas

Ciudad de México, 24 de Julio del 2019 

Espacio Recíproco

Es fácil encogerse frente a la complejidad del momento en que vivimos, frente a la monotonía que encontramos dentro y fuera de nuestras celdas. Quizá la falta de armónica simetría enrarece nuestras vecindades, y nos lleva a buscar periodicidad en la amorfa realidad, incluso a costa de nuestros grados de libertad. Pero no somos unidimensionales, ni monótonos. Aceptar nuestra irregularidad, abrazar la incertidumbre, es un paso importante en la búsqueda de mínimos de ansiedad y máximos de satisfacción. Entender y perder el

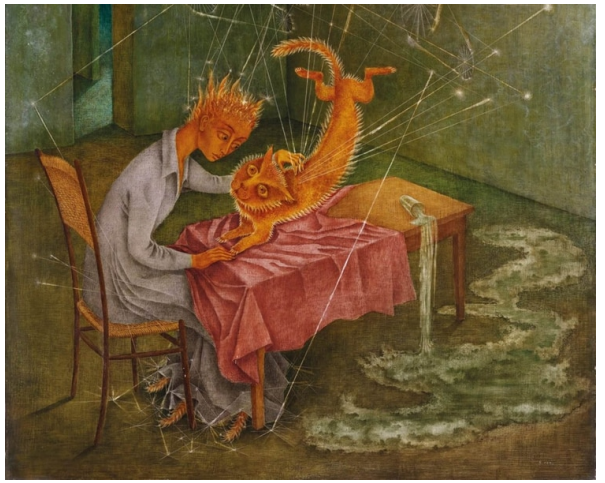

Simpatía (La rabia del gato). Remedios Varo, 1955. temor al espacio ajeno puede mejorar las condiciones en las que nuestro espacio personal existe. Aumentar nuestro panorama no solo nos dará libertad, disminuirá en forma recíproca la complejidad con que observamos el mundo ajeno. Disfrutemos del espacio, en todo momento.

A Yunuen, mi compañera de viaje y de celda. 



\section{Agradecimientos}

Siempre como base de todos mis logros, agradezco a mis padres, Elizabeth y Marco, por todos sus esfuerzos para llevar a flote a mi y a mis hermanos.

A mi esposa Yunuen, por la compañía y ternura que me ha brindado estos años. Por serenar mis momentos de mayor ansiedad.

Agradezco a mi asesor Dr. Jorge Garza, por su guía y apoyo para poder culminar esta etapa, además de la motivación para seguir adelante, con el ejemplo que para mí representa.

Muchas gracias a la Dra. Rubicelia Vargas, por ayudarme a recuperar el sentido de mi carrera y por su gran amabilidad.

A mis sinodales, por el tiempo que dedicaron a la revisión de este escrito y las observaciones que permitieron mejorarlo.

A mis amigos de carrera: Julio, Juan, Daniel, Gabriel y Omar, por su compañía y apoyo, por retarme a seguirles el paso.

Agradezco al Laboratorio de Supercómputo de la UAM-I, por el tiempo de cómputo otorgado, y al CONACyT por el apoyo mediante la beca de maestría 633579. 


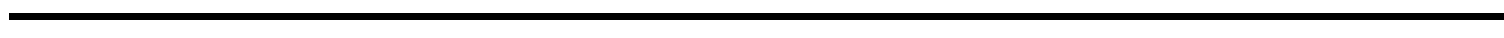




\section{Productos}

Parte de los resultados obtenidos fueron presentados en la XVII Reunión Mexicana de Fisicoquímica Teórica, en Monterrey, Nuevo León, en Noviembre de 2018, con el póster titulado Evaluación de integrales bielectrónicas sobre GPUs en sistemas periódicos.

Por otro lado, se publicaron los siguientes artículos, mostrados en el Apéndice B:

- Navarrete-López, A. M.; Rivera-Almazo, M.; Garza, J. y Vargas, R. Importance of one-parameter hybrid exchange-correlation functionals in band gaps of transition metal and metalloid oxides. Theor. Chem. Acc. 2018, 137 (3).

- Alcaraz, F. A. D.; Martinez-Sanchez, M.-A.; Rivera-Almazo, M.; Vargas, R.; Rosas-Burgos, R. A. y Garza, J. Testing one-parameter hybrid exchange functionals in confined atomic systems. J. Phys. B 2019, 52 (13). 

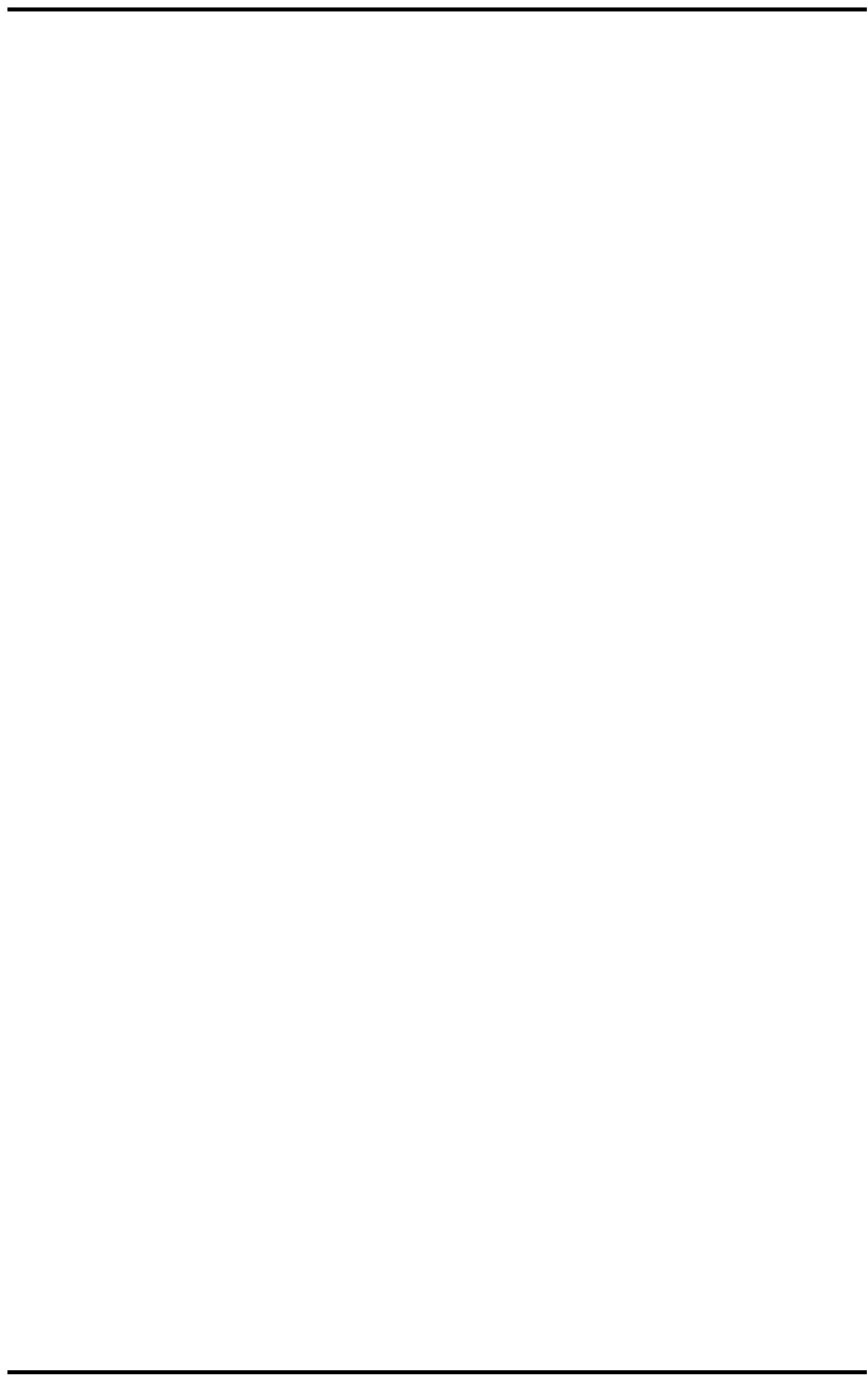


\section{Índice general}

Agradecimientos

Productos $\quad$ V

1. Resumen 1

2. Introducción 3

$\begin{array}{lll}\text { 3. Intercambio electrónico } & 7\end{array}$

3.1. Intercambio en el método de Hartree-Fock . . . . . . . . . . . . 7

3.2. Teoría de funcionales de la densidad . . . . . . . . . . . . 10

3.2.1. Método de Kohn-Sham . . . . . . . . . . . . . . 11

3.2.2. Funcionales de intercambio y correlación híbridos . . . . . 13

4. Funciones localizadas en el

$\begin{array}{ll}\text { estudio de sistemas cristalinos } & 16\end{array}$

4.1. Estructura electrónica de sólidos . . . . . . . . . . . . . . . 16 
4.2. Construcción de Orbitales Cristalinos . . . . . . . . . . . . . 18

4.2.1. El programa CRYSTAL . . . . . . . . . . . . . 19

4.3. Construcción de las matrices . . . . . . . . . . . . . . 20

4.3.1. La Matriz densidad . . . . . . . . . . . . . . . . . 21

4.3.2. La Matriz de Fock . . . . . . . . . . . . . . . 22

4.4. Tratamiento de las sumas infinitas . . . . . . . . . . . . 25

4.4.1. Estrella de vectores ... . . . . . . . . . . . 29

4.4.2. Evaluación de las integrales bielectrónicas . . . . . . . . . 30

4.5. Algoritmo del código . . . . . . . . . . . . . . . 31

4.5.1. Programa principal ................ 31

4.5.2. Método de campo autoconsistente . . . . . . . . . . 32

4.5.3. Selección y evaluación de las integrales bielectrónicas . . . . 33

4.6. Cómputo heterogéneo . . . . . . . . . . . . 36

4.6.1. Dispositivos de procesamiento actuales . . . . . . . . 37

4.6.2. Algunas herramientas para cómputo heterogéneo . . . . . . . 40

4.7. CRYSTAL sobre cómputo heterogéneo . . . . . . . . . . . . . 41

4.7.1. Pruebas realizadas ................ . . . 42

4.7.2. Perspectivas ..................... 52

\section{Efecto del intercambio exacto}


5.1. Metodología ....................... 54

5.2. Parámetros geométricos y Módulo de Bulto . . . . . . . . . . 57

5.3. Estructura de bandas y densidad de estados . . . . . . . . . . . 59

5.4. Band gap ............................ 65

5.5. Conclusiones ...................... 67

5.6. Perspectivas ......................... 68

$\begin{array}{ll}\text { A. Detalles del uso de OpenACC } & 70\end{array}$

$\begin{array}{ll}\text { B. Artículos publicados } & 75\end{array}$ 


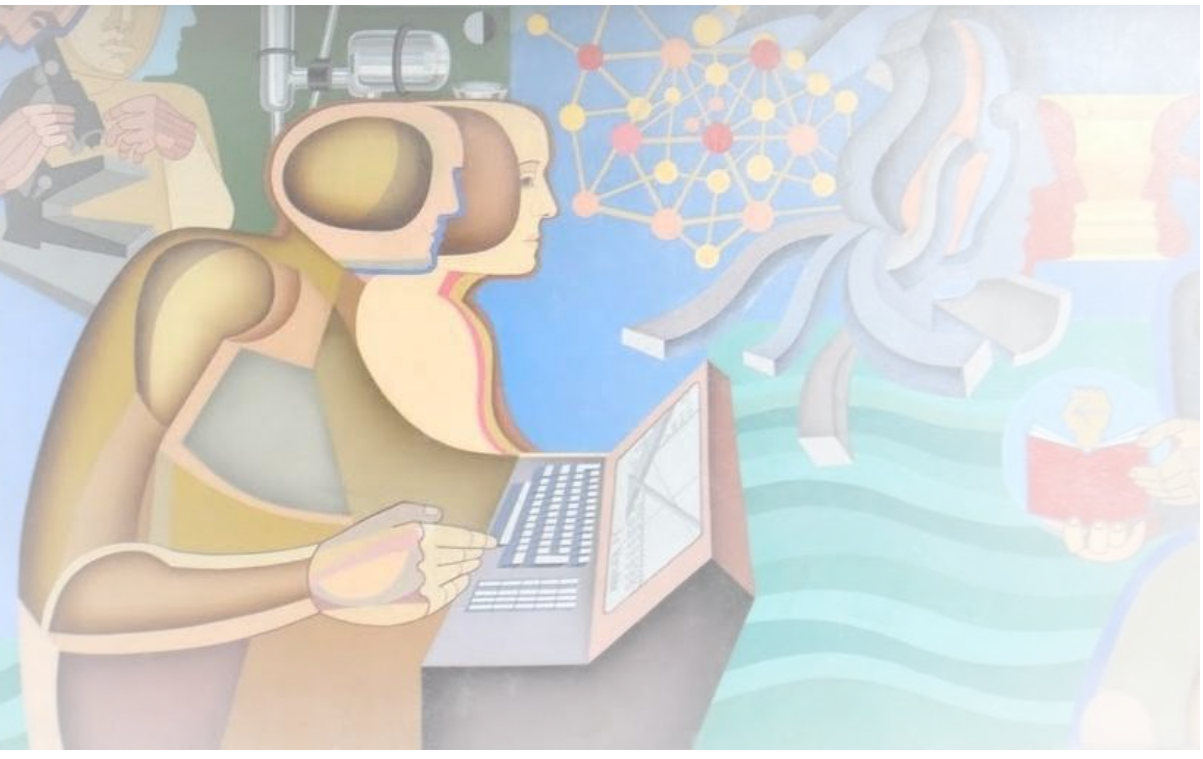

\section{Resumen}

Algunos óxidos de metales de transición y de metaloides presentan valores de band gap adecuados para su uso en aplicaciones tales como catálisis y celdas solares. Desafortunadamente, algunas aproximaciones teóricas usadas para describir estos sistemas, como el uso de funcionales de intercambio y correlación tipo LDA y GGA en Teoría de Funcionales de la Densidad bajo el marco de Kohn-Sham, suelen predecir incorrectamente el valor del band gap. El uso de funcionales híbridos, que incluyen una fracción de intercambio exacto, puede mejorar el cálculo de esta propiedad si la fracción adecuada de dicha contribución es elegida.

En esta tesis se muestra un estudio del efecto del intercambio exacto en distintos óxidos de metales de transición y metaloides. Los cálculos realizados para dicho fin fueron realizados usando el programa CRYSTAL, el cual permite estudiar sistemas periódicos utilizando funciones localizadas como base. En una primera parte se describe la base teórica de la contribución de intercambio. Después, se muestran los fundamentos del código CRYSTAL, con énfasis en la resolución de las integrales bielectrónicas, analizando además los beneficios que implicaría su uso sobre cómputo heterogéneo y cuales son las condiciones para que esto pueda ocur- 
rir. Finalmente, se analizan los resultados obtenidos sobre el efecto de la fracción de intercambio exacto sobre el band gap en los sistemas estudiados, mostrando que un valor adecuado de intercambio conserva buenos resultados para las propiedades geométricas y mecánicas, y que el funcional GGA PBE describe de forma incorrecta estos sistemas. 

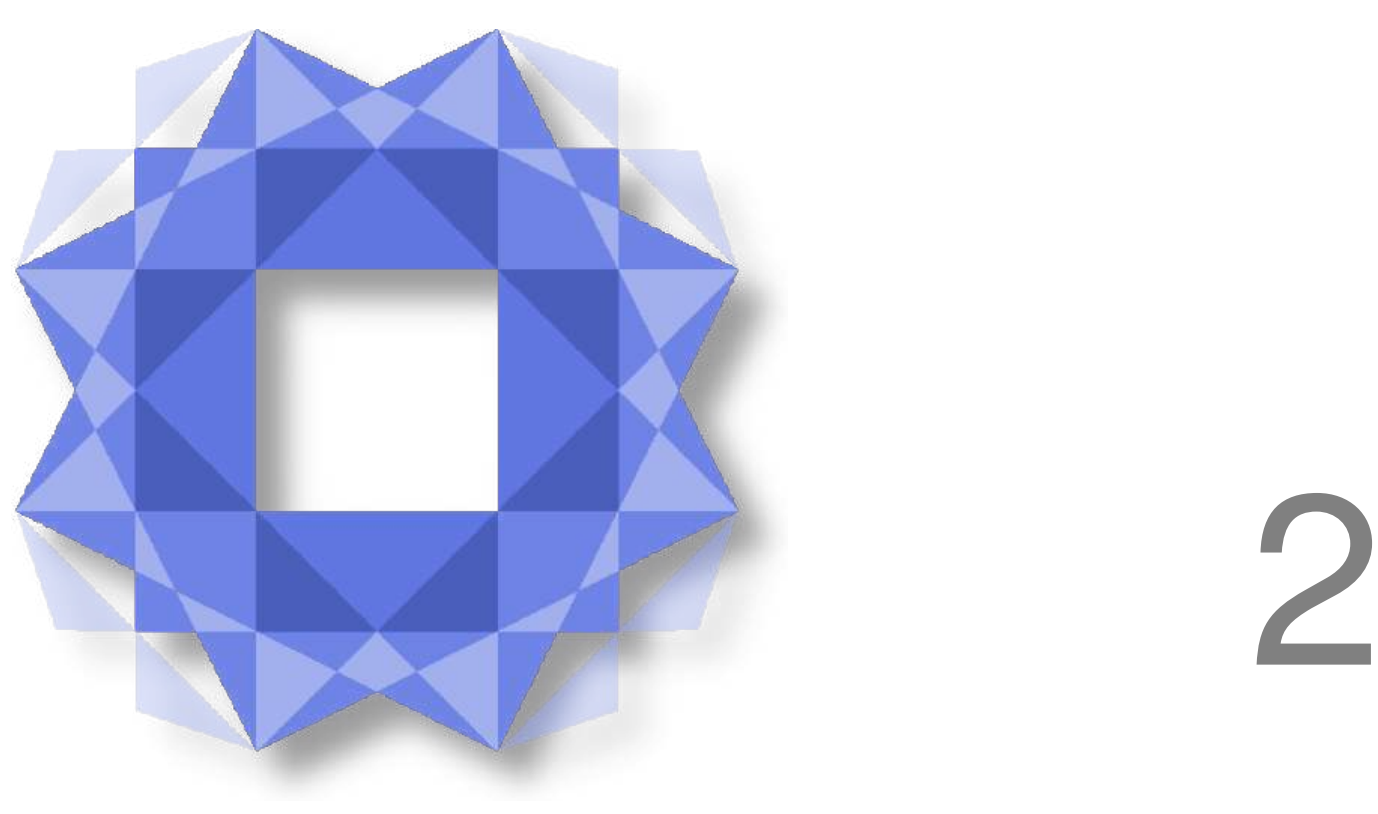

\section{Introducción}

Los óxidos de metales de transición son de gran interés en el desarrollo de nuevos materiales, dada la amplia variedad de fenómenos electrónicos que pueden presentar, abarcando ferro y antiferromagnetismo, catálisis, superconductividad, fenómenos dieléctricos, ferroeléctricos, termoeléctricos, multiferroicidad, y transiciones metal-aislante, por mencionar algunos.[1] Por la variedad de sus aplicaciones estos compuestos se han aprovechado en materiales catalíticos para la conversión de hidrocarburos, en electrodos, para películas conductoras, para celdas solares, y como superconductores de altas temperaturas.[2,3] En algunas de estas aplicaciones resulta importante el valor del band gap que estos sistemas y sus derivados presentan,[4] al determinar bajo que condiciones estos materiales pueden conducir electrones.

Varias de las propiedades únicas de estos sistemas son adjudicadas a la fuerte correlación que presentan los electrones $d$ de valencia en los iones metálicos de estos óxidos.[5] Es también por esta razón que ha resultado difícil la descripción correcta de algunas de sus propiedades físicas mediante los modelos teóricos más utilizados. Muchos de los cálculos realizados en química computacional, bajo la 
Teoría de Funcionales de la Densidad en el marco de Kohn-Sham, utilizan funcionales de intercambio y correlación tipo Aproximación de Densidad Local y Aproximación de Gradiente Generalizado (LDA y GGA respectivamente, por sus siglas en ingles), dado su bajo costo computacional y el éxito que han tenido en la descripción teórica de varios sistemas. Sin embargo, ambos métodos no describen correctamente la estructura de bandas de los óxidos de metales de transición; en el caso del valor del band gap, estos cálculos suelen subestimar el valor de esta propiedad, llegando inclusive a predecir como conductores a sistemas que son aislantes.[6-8]

Por otro lado, los óxidos de elementos del grupo IVA, como el $\mathrm{SiO}_{2}$, el $\mathrm{GeO}_{2}$ y el $\mathrm{SnO}_{2}$, son también de gran interés tecnológico, presentando una variedad de propiedades útiles en el diseño de celdas solares y dispositivos electrónicos.[9] Al igual que con los óxidos de metales de transición, los métodos tipo LDA y GGA no predicen correctamente la información experimental de estos sistemas, subestimando también el valor del band gap.[9, 10]

Existen reportes en donde el uso de potenciales de intercambio y correlación que incluyen una fracción de intercambio exacto mejoran la descripción del band gap de varios sistemas.[11, 12] Los funcionales conocidos como híbridos contienen una contribución de intercambio exacto, utilizando uno o más parámetros para el control de dicho término. Uno de los funcionales de este tipo más utilizados, el funcional PBE0, incluye una fracción de 1/4 de intercambio exacto bajo fundamentos teóricos justificados, describiendo adecuadamente una gran cantidad de sistemas finitos. Sin embargo, en ocasiones este funcional no describe correctamente propiedades de sistemas periódicos, llegando a sobrestimar frecuencias vibracionales $[13,14]$ y arrojando valores incorrectos del band gap en algunos sistemas con orbitales $d$.[15] Existen otras propuestas para la contribución del intercambio exacto en el funcional PBE0, resultando en mejores resultados para sistemas de naturaleza específica.[16-18] 
Por otro lado, se sabe que entre el band gap y la fracción de intercambio exacto de un funcional híbrido existe un comportamiento lineal.[19] Esta relación puede utilizarse para ajustar la contribución de intercambio exacto en estos funcionales, de tal forma que el band gap coincida con el gap experimental. En específico, cuando el funcional híbrido utiliza un solo parámetro para establecer la fracción a considerar de intercambio exacto, como en el caso de PBE0, resulta directo el ajuste de esta contribución con dicho fin.

\section{Objetivos}

Objetivo 1

Dados estos antecedentes, el objetivo principal de esta tesis es determinar, en el caso de algunos óxidos de metales de transición y de elementos del grupo IVA, cual es la fracción adecuada de intercambio exacto en un funcional basado en PBE0 tal que se reproduzca el valor experimental del band gap, estableciendo además si el uso de dicha fracción describe correctamente los parámetros geométricos y las propiedades mecánicas de estos sistemas. Para cumplir este objetivo se realizaron cálculos ab initio de estructura electrónica de sistemas periódicos usando el código CRYSTAL, el cual utiliza como base funciones localizadas tipo gaussianas. La contribución de intercambio exacto en CRYSTAL, al igual que la interacción coulómbica electrón-electrón, involucra el cálculo de integrales bielectrónicas, conocidas por su alto costo computacional tanto en sistemas finitos como en sistemas periódicos. Muchos de los sistemas de interés tecnológico requieren de un gran poder computacional para poder ser estudiados teóricamente con funcionales híbridos, lo cual puede limitar bastante en algunos centros de investigación el nivel de teoría y la naturaleza de los sistemas que pueden explorar. 


\section{Objetivo 2}

Por estas razones, otro objetivo de este trabajo es la búsqueda de técnicas computacionales que aceleren la ejecución de CRYSTAL, aprovechando para dicho fin los sistemas heterogéneos de cómputo que en la actualidad existen. En específico, se muestran esfuerzos para ejecutar CRYSTAL aprovechando por un lado GPUS y por otro procesadores tipo POWER, estableciendo cuáles son algunos de los requisitos necesarios para que este programa aproveche estas tecnologías. 


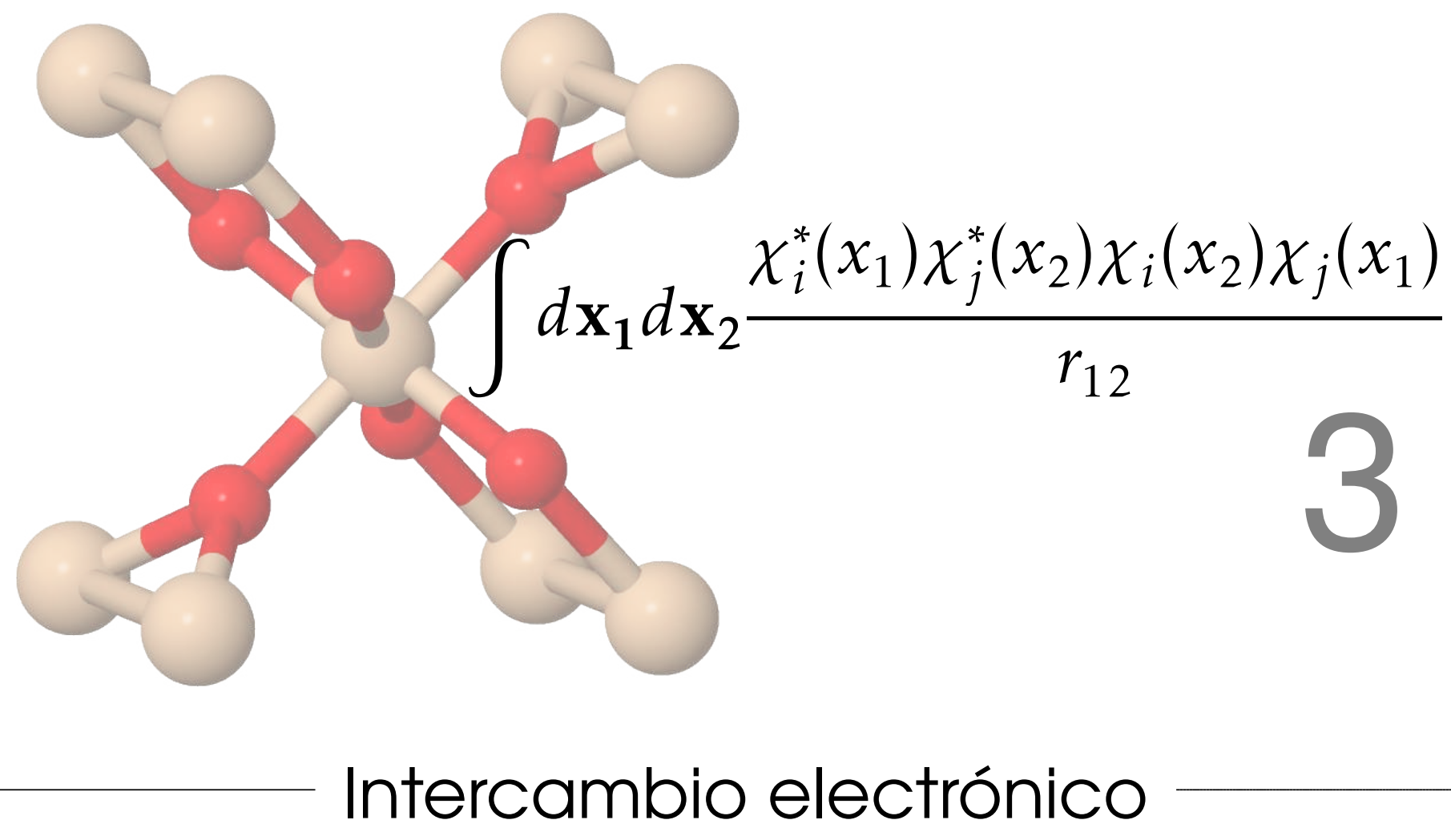

3.1 Intercambio en el método de Hartree-Fock

Los estudios computacionales de sistemas químicos a un nivel cuántico tienen como base la resolución de la ecuación de Schrödinger,[20]

$$
\hat{H}(\mathbf{r}) \Psi(\mathbf{r})=E \Psi(\mathbf{r})
$$

con lo cual se puede obtener una descripción de la estructura electrónica de sistemas moleculares. Dependiendo de la metodología utilizada para resolver la ecuación de Schrödinger los resultados pueden variar en precisión e inversamente en costo computacional. El método que facilitó el estudio de sistemas multielectrónicos fue el de Hartee-Fock (HF) y de éste se desprendieron otros métodos conocidos como Post-Hartree-Fock. En el caso del método HF se sabe que existe una diferencia entre la energía obtenida y la exacta ${ }^{1}$, siendo llamada esta diferencia como energía de correlación. Los métodos Post-HF intentan incluir la energía de cor-

\footnotetext{
${ }^{1}$ Energía electrónica exacta no relativista bajo la aproximación de Born-Oppenheimer
} 
relación, pero el costo computacional de dichos métodos impide que puedan ser utilizados para estudiar sistemas con decenas de átomos o cientos de electrones sobre infraestructuras con decenas de procesadores.[21]

En estos métodos un elemento clave es el uso de determinantes de Slater para escribir a la función de onda. Dicho determinante está compuesto por espín-orbitales $\chi$, los cuales se construyen como el producto de un orbital espacial $\varphi(\mathbf{r})$ y una función de espín $\sigma(\omega)$ que depende de la coordenada de espín $\omega$, tal que $\chi(\mathbf{x})=$ $\varphi(\mathbf{r}) \sigma(\omega)$, con $\mathbf{x}$ representando al conjunto de coordenadas $\mathbf{r}$ y $\omega$. Usando operadores de permutación, el determinante de Slater puede escribirse cómo:

$$
\Psi^{\text {Slater }}=\frac{1}{\sqrt{N !}} \sum_{n=1}^{N !}(-1)^{P_{n}} \mathfrak{P}_{\mathfrak{n}} \chi_{i}(1) \chi_{j}(2) \ldots \chi_{k}(N),
$$

donde $N$ es el número de electrones, $\mathfrak{P}_{\mathfrak{n}}$ es el operador de permutación, $P_{n}$ es el número de transposiciones necesarias para regresar de una permutación dada al estado original, y $\chi_{i}(1)$ es el espín-orbital $i$ evaluado en las coordenadas del electrón 1. En esta expresión se aprecia que el determinante está compuesto por una combinación de productos de espín-orbitales, donde en cada término de la suma las coordenadas se encuentran intercambiadas por acción del operador de permutación.

Una consecuencia de usar un determinante de Slater es que la probabilidad de encontrar dos electrones con espín paralelo en un mismo punto en el espacio es cero. Dado que este efecto esta asociado al intercambio de coordenadas del determinante se le conoce como correlación de intercambio o simplemente intercambio.

Bajo la aproximación de HF, que utiliza un solo determinante de Slater para $\Psi$, la energía total del sistema esta descrita por:

$$
E_{H F}=\sum_{i}^{N}\langle i|\hat{h}| i\rangle+\frac{1}{2} \sum_{i}^{N} \sum_{j}^{N}\langle i j \mid i j\rangle-\langle i j \mid j i\rangle,
$$


donde $i$ y $j$ son espín-orbitales, y $\hat{h}$ es el operador monoelectrónico $\left(\hat{h}=-\frac{1}{2} \nabla^{2}+\right.$ $v_{n e}(\mathbf{r})$, con $v_{n e}$ el operador de interacción núcleo-electrón). En el primer término de la ecuación (3.3), $\langle i|\hat{h}| i\rangle$ representa la integral monoelectrónica,

$$
\langle i|\hat{h}| i\rangle=\int d \mathbf{x} \chi_{i}^{*}(\mathbf{x})\left(-\frac{1}{2} \nabla^{2}+v_{n e}(\mathbf{r})\right) \chi_{i}(\mathbf{x})
$$

mientras que en el segundo término $\langle i j \mid i j\rangle$ y $\langle i j \mid j i\rangle$ corresponden a integrales bielectrónicas con la forma:

$$
\langle i j \mid i j\rangle=\int d \mathbf{x}_{1} d \mathbf{x}_{2} \chi_{i}^{*}\left(\mathbf{x}_{1}\right) \chi_{j}^{*}\left(\mathbf{x}_{2}\right) r_{12}^{-1} \chi_{i}\left(\mathbf{x}_{1}\right) \chi_{j}\left(\mathbf{x}_{2}\right) .
$$

La primer integral bielectrónica del término enmarcado en (3.3) está conectada con la interacción electrostática entre las densidades electrónicas de dos electrones y como tal se le conoce como integral coulómbica, mientras que el segundo es similar pero con un intercambio de coordenadas en los espín-orbitales no conjugados, conociéndose consecuentemente como integral de intercambio. Si se integra sobre las coordenadas de espín para reducir las expresiones a orbitales espaciales $\varphi, y$ considerando un sistema de capa cerrada, la energía puede escribirse como:

$$
E_{H F}=2 \sum_{i}\langle i|\hat{h}| i\rangle+\sum_{i j} 2\langle i j \mid i j\rangle-\langle i j \mid j i\rangle=2 \sum_{i} h_{i i}+\sum_{i j} 2 J_{i j}-K_{i j},
$$

donde ahora $i$ y $j$ corresponden a orbitales espaciales, $h_{i i}$ corresponde a integrales monoelectrónicas, y $J_{i j}$ y $K_{i j}$ son las integrales coulómbicas y de intercambio respectivamente. Una vez evaluadas las integrales, es posible agrupar las respectivas contribuciones a la energía total e identificar la energía debida al intercambio. Es importante destacar que la presencia de los términos de intercambio en (3.6) cancela los términos de autointeracción coulómbica, donde $i=j$.

Dentro del método de HF al final terminamos con ecuaciones monoelectróni- 
cas, de la forma:

$$
\hat{f} \varphi_{i}=\left(\hat{h}+\sum_{j} \hat{J}_{j}-\hat{K}_{j}\right) \varphi_{i}=\varepsilon_{i} \varphi_{i},
$$

donde el operador de Fock, $\hat{f}$, contiene las contribuciones de interacción electrónelectrón coulómbica y de intercambio, además de las contribuciones monoelectrónicas. Los operadores bielectrónicos y de interacción núcleo-electrón pueden agruparse para formar un operador de potencial efectivo:

$$
v_{e f f}^{H F}=v_{n e}(\mathbf{r})+\sum_{j} \hat{J}-\hat{K} .
$$

Dado que la expresión que se tiene para el fenómeno de intercambio en el método de Hartree-Fock es exacta para un determinante de Slater, permitiendo la cancelación completa de la autointeracción electrón-electrón, en la metodología que se describirá más adelante se le llamará a esta contribución intercambio exacto. Dado que en dicho caso no se utilizan los orbitales de HF se evitará llamarle intercambio de HF, puesto que dicha contribución es en general distinta a la obtenida en el método de HF.

\subsection{Teoría de funcionales de la densidad}

Una alternativa a los métodos basados en la función de onda es utilizar como variable central la densidad electrónica $\rho$, definida como:[22]

$$
\rho(\mathbf{r})=N \int \cdots \int d \omega_{1} d \mathbf{x}_{1} d \mathbf{x}_{2} \ldots d \mathbf{x}_{\mathbf{N}}\left|\Psi\left(\mathbf{x}_{1}, \mathbf{x}_{2}, \ldots, \mathbf{x}_{\mathbf{N}}\right)\right|^{2},
$$

done $\mathbf{x}_{\mathbf{i}}$ comprende tanto las coordenadas espaciales $\mathbf{r}$ como la coordenada de espín $\omega$ del electrón $i$. La integral múltiple en la ecuación representa la probabilidad de encontrar el electrón 1 en $d \mathbf{r}_{1}$; dada la indistinguibilidad de los electrones, la probabilidad de encontrar cualquier electrón en dicho volumen es $N$ veces la pro- 
babilidad de encontrar a este electrón en particular, por lo que $\rho$ depende de una r no asociada a algún electrón en particular.

La Teoría de Funcionales de la Densidad (DFT) se basa en que un sistema puede ser caracterizado a partir de su densidad electrónica. Esta teoría tiene sustento básico en los teoremas de Hohenberg y Kohn, que establecen por un lado la existencia de una densidad electrónica basal única para un potencial externo dado y por otro la existencia de un principio variacional, donde la energía obtenida con una densidad electrónica aceptable ${ }^{2}$ no puede ser menor a la obtenida con la densidad del estado basal. Del primer teorema se tiene que una densidad electrónica corresponde a una función de onda, y es posible entonces describir el sistema utilizándola en lugar de $\Psi$.

La energía total del sistema en términos de la densidad electrónica, dados los teoremas mencionados, tiene la siguiente forma funcional:

$$
E[\rho]=F[\rho]+\int d \mathbf{r} v_{e x t}(\mathbf{r}) \rho(\mathbf{r})=T[\rho]+J[\rho]+E_{n c l}[\rho]+V_{\text {ext }}[\rho]
$$

donde el funcional $F$ es conocido como funcional universal, ya que su forma es independiente el sistema a tratar. Este funcional puede separarse en los funcionales de energía cinética, $T$, de interacción coulómbica electrón-electrón, $J$, y del resto de contribuciones no clásicas de interacción electrón-electrón, $E_{n c l}$. La forma tanto del funcional $T$ como el $E_{n c l}$ es desconocida, por lo que si se desea utilizar esta relación es necesario utilizar alguna aproximación a ambos.

\subsubsection{Método de Kohn-Sham}

La forma más extendida en la que se aplica la DFT es usando el método de Kohn-Sham (KS). En este método la densidad electrónica se aproxima como aque-

\footnotetext{
${ }^{2}$ Que cumpla con ser positiva, al integrarse sobre todo el espacio dé el número de electrones, y esté asociada a un potencial externo.
} 
lla que se tiene para un sistema de partículas independientes, es decir, la correspondiente a un determinante de Slater:

$$
\rho_{S}(\mathbf{r})=\sum_{i}^{N} \sum_{\omega}\left|\chi_{i}^{K S}(\mathbf{x})\right|^{2},
$$

donde se hace énfasis en que los espín-orbitales corresponden al método de KS y no a los de HF.

Bajo KS la energía total del sistema se escribe como:

$$
E[\rho]=T_{s}[\rho]+J[\rho]+E_{X C}[\rho]+V_{\text {ext }}[\rho]
$$

donde el funcional $T_{S}$ corresponde a la energía cinética del sistema de partículas independientes, tal como se escribe en HF, y $E_{X C}$ se conoce como funcional de intercambio y correlación.

El funcional $E_{X C}$ contiene las contribuciones no clásicas de interacción entre electrones y el resto de la energía cinética, dado que $T_{s}$ no corresponde a la energía cinética del sistema real. Al igual que con el funcional universal, se desconoce actualmente la forma exacta de $E_{X C}$, y son utilizadas diversas aproximaciones para usar el método. El nombre de este funcional está ligado a la nomenclatura utilizada en el método de HF, ya que contempla la energía no incluida en el resto de los términos, como la energía de correlación en HF, y es conocida la existencia de una contribución ligada al fenómeno del intercambio.

El costo computacional de resolver las ecuaciones involucradas en KS es razonable en la mayoría de los casos, escalando aproximadamente como $M^{3}$ o $M^{4}$ con M el tamaño de la base utilizada, de forma similar a como escala HF.[23] El beneficio que se obtiene frente a HF usando el método de KS es que es posible incluir aproximaciones a la energía de correlación, mejorando en muchos de los casos los resultados obtenidos. 
Manipulando adecuadamente la expresión (3.12) es posible obtener ecuaciones orbitales como en HF:

$$
\hat{f}^{K S} \varphi_{i}=\left(-\frac{1}{2} \nabla^{2}+v_{e f f}^{K S}\right) \varphi_{i}=\varepsilon_{i} \varphi_{i}
$$

Dado esto, podemos escribir de forma general la siguiente ecuación:

$$
\left(-\frac{1}{2} \nabla^{2}+v_{e f f}\right) \varphi_{i}=\varepsilon_{i} \varphi_{i}
$$

donde el potencial efectivo y los orbitales pueden ser los de HF o los de KS según sea el caso.

\subsubsection{Funcionales de intercambio y correlación híbridos}

Existen distintos métodos utilizados para desarrollar aproximaciones de $E_{X C}$. Según Perdew,[24] añadir ciertas contribuciones al funcional puede mejorar su precisión, partiendo de la densidad local y continuando con el gradiente de la misma. De esta consideración puede clasificarse al funcional como de Aproximación de la Densidad Local (LDA) si solo incluye $\rho$, o de Aproximación de Gradiente Generalizado (GGA) si además se incluye $\nabla \rho$.

Otra posibilidad es incluir una contribución de intercambio exacto IEX. Este tipo de funcional es conocido como híbrido, al incluir tanto una contribución de intercambio GGA como de intercambio exacto. Existen actualmente distintas formas en las que se añade al funcional esta contribución. Una propuesta de Becke para este tipo de funcional tiene la siguiente forma:[25]

$$
E_{X C}^{\text {hybrid }}=E_{X C}^{\text {aprox }}+\alpha\left(I E X-E_{X}^{\text {aprox }}\right),
$$

donde $E_{X}$ y $E_{C}$ suelen ser funcionales tipo GGA y el parámetro $\alpha$ determina la 
fracción a considerar de intercambio exacto, disminuyendo consecuentemente el intercambio GGA. Entre los funcionales híbridos más utilizados que siguen esta forma están aquellos basados en el funcional de intercambio y correlación PBE, el cual pertenece a la familia de funcionales GGA. En el funcional PBE0, donde el cero denota la ausencia de parámetros empíricos, el valor de $\alpha$ es determinado como 0.25.[26, 27] PBE0 ha sido usado de forma recurrente en estudios de diversos sistemas, pero en ciertos casos, como en el de sistemas periódicos con elementos pesados, no brinda una descripción adecuada. Por este motivo se ha reportado el uso de otros valores de $\alpha$ distintos, como $1 / 2,1 / 3$ y 1/5,[16-18] para mejorar la descripción de ciertos tipos de sistemas.

Dado que el valor adecuado de $\alpha$ varía dependiendo de la naturaleza del sistema, han sido propuestas metodologías para ajustar el intercambio exacto en algunos funcionales híbridos. Varios trabajos modifican en este sentido los funcionales de la familia HSE, que son funcionales híbridos de rango separado y con dos contribuciones distintas de IEX: una de rango corto y otra de rango largo. En estos estudios suelen utilizar una constante dieléctrica, $\epsilon$, para estimar la fracción a utilizar de IEX. $[7,28]$ Uno de estos trabajos encontró que existe una relación lineal entre el band gap calculado y el parámetro $\alpha$ de estos funcionales,[19] mientras que en otro se observa que la relación entre otro de los parámetros del funcional y el valor de $\epsilon$ también es lineal.[29] En este último trabajo se muestra que existe una infinidad de combinaciones posibles de los parámetros de los funcionales HSE que permiten obtener un valor de band gap. Recientemente se han encontrado metodologías donde ajustan la contribución de IEX de forma autoconsistente,[30, 31] o tratan de deducirla a partir de primeros principios. [32]

Siendo este un tema de continua discusión en los años recientes, es de interés para este trabajo explorar los efectos del intercambio exacto en cálculos TFD en un grupo de óxidos metálicos. Se busca entonces estudiar cual es la fracción de IEX en un funcional híbrido basado en PBE adecuada para reproducir el band gap experimental de cada sistema, y verificar que en el uso de dicha fracción otras propie- 
dades de importancia siguen en buen acuerdo con la información experimental. Durante este proceso, esperamos encontrar tendencias que puedan ayudar en la búsqueda y mejoramiento de metodologías para el ajuste del parámetro. 

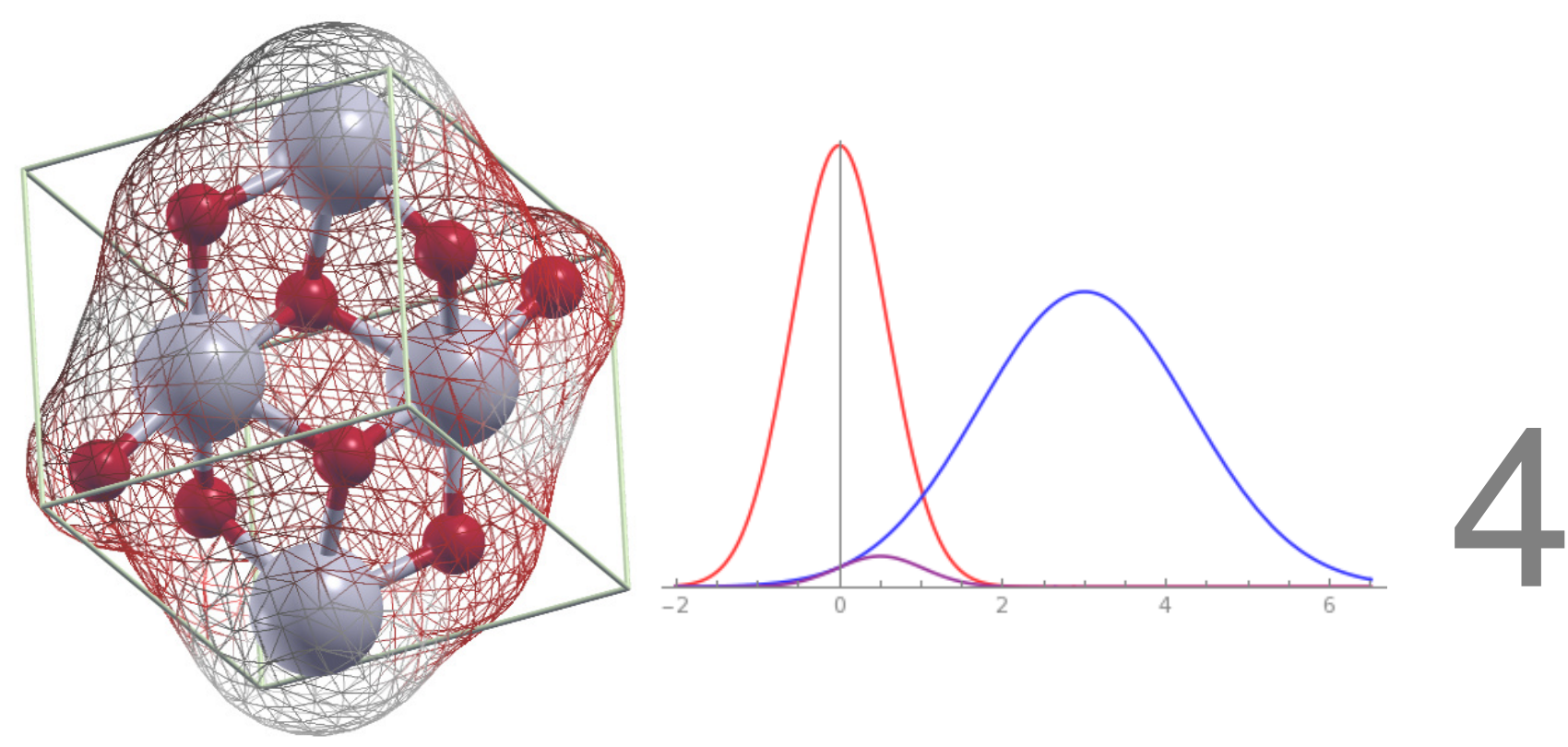

\section{Funciones localizadas en el}

\section{estudio de sistemas cristalinos}

\subsection{Estructura electrónica de sólidos}

Los sistemas involucrados en este trabajo fueron estudiados en su fase cristalina. Con el fin de estudiar la estructura electrónica de un cristal, siendo éste un arreglo de átomos o moléculas en una malla ordenada e infinita de puntos, es necesario resolver la ecuación de Schrödinger bajo ciertas consideraciones. Dada la periodicidad del sistema es de esperarse que el potencial $V(\mathbf{r})$ en un punto $\mathbf{r}$ tenga la misma periodicidad que el cristal. Dado este efecto de la simetría translacional, la ecuación de Schrödinger asociada (ecuación 3.1) tendrá las mismas soluciones que las obtenidas cuando el sistema es trasladado por un vector de la malla $\mathbf{g}$ :[33]

$$
\hat{H}(\mathbf{r}+\mathbf{g}) \Psi(\mathbf{r}+\mathbf{g})=\hat{H}(\mathbf{r}) \Psi(\mathbf{r}) E \Psi(\mathbf{r}+\mathbf{g}),
$$

con $\mathbf{g}$ un vector de la malla en el espacio real (Figura 4.1a). Dada la periodicidad del potencial, se cumple que $\hat{H}(\mathbf{r}+\mathbf{g})=\hat{H}(\mathbf{r})$, de donde es claro que ambos Hamil- 
tonianos comparten las mimas funciones y valores propios.

Las funciones propias que satisfacen dicha condición, asociadas por tanto a un potencial periódico, cumplen el teorema de Bloch:

$$
\phi(\mathbf{r}+\mathbf{g} ; \mathbf{k})=e^{i \mathbf{k} \cdot \mathbf{g}} \phi(\mathbf{r} ; \mathbf{k}),
$$

donde $\phi$ se conoce como función de Bloch, y es función del vector de posición $\mathbf{r}$ y el vector de onda $\mathbf{k}$; para un vector $\mathbf{k}$ se tiene una solución de la ecuación 4.1. Si se sustituye (4.2) en (4.1) se recupera la ecuación de Schrödinger (ecuación 3.1).

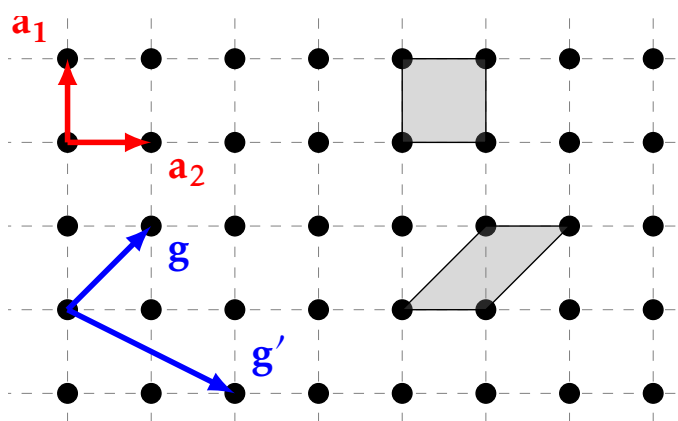

(a) Espacio real

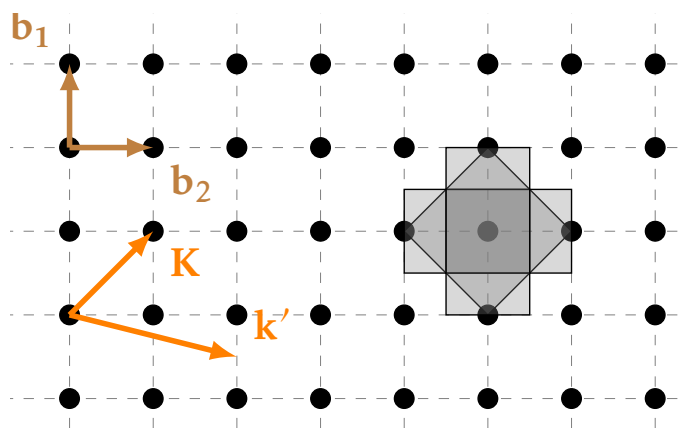

(b) Espacio recíproco

Figura 4.1: Malla cuadrada en distintos espacios. Los vectores $\mathbf{a}_{1}, \mathbf{a}_{2}, \mathbf{b}_{1}$ y $\mathbf{b}_{2}$ son unos de los posibles vectores base en sus respectivos espacios; los vectores $\mathbf{g}$, $\mathbf{g}^{\prime}$ y $\mathbf{K}$ son combinaciones lineales de éstos, mientras que $\mathbf{k}$ es un punto cualquiera en el espacio recíproco. Las zonas sombreadas en (a) son celdas unitarias. Las zonas sombreadas en (b) representan las primeras tres zonas de Brillouin; la primer zona de Brillouin es la más oscura.

Es posible establecer que $\mathbf{k}$ es un punto en la malla recíproca del cristal (Figura $4.1 \mathrm{~b})$ y que la cantidad de estos puntos es infinita, con lo cual puede considerarse que $\mathbf{k}$ es una variable continua. Si en una función de Bloch le sumamos a $\mathbf{k}$ un vector de la malla $\mathbf{K}$ se puede comprobar que el resultado es equivalente al de la ecuación 4.2, por lo cual esta función es aceptada como función propia asociada al mismo punto $\mathbf{k}$. Dado este comportamiento es posible restringir el análisis de las soluciones de la ecuación de Schrödinger a una de las zonas de Brillouin; en general se elige por conveniencia la primera zona de Brillouin.

Ya que las funciones de Bloch son funciones propias de operadores de traslación 
y de aquellos operadores con los que estos conmutan, como el operador Hamiltoniano del cristal, estas funciones son bases de la representaciones irreducibles del grupo de las traslaciones del cristal, con un punto $\mathbf{k}$ asociada a cada representación. De la teoría de grupos se sabe que las funciones de base que pertenecen a distintas representaciones irreducibles son ortogonales entre ellas; si utilizamos $n$ funciones de Bloch como base para resolver la matriz Hamiltoniana, que es infinita como nuestro sistema, ésta será una matriz infinita de bloques diagonales, cuyos bloques están asociados a un punto k en particular (Figura 4.2).

La matriz Hamiltoniana de bloques diagonales así obtenida se resuelve para un conjunto finito y específico de puntos $\mathbf{k}$, que en general se encuentran en la primera zona de Brillouin. Para cada punto $\mathbf{k}$ se resuelve entonces la ecuación:

$$
\hat{H} \Psi_{n}(\mathbf{r} ; \mathbf{k})=E_{n}(\mathbf{k}) \Psi_{n}(\mathbf{r} ; \mathbf{k})
$$
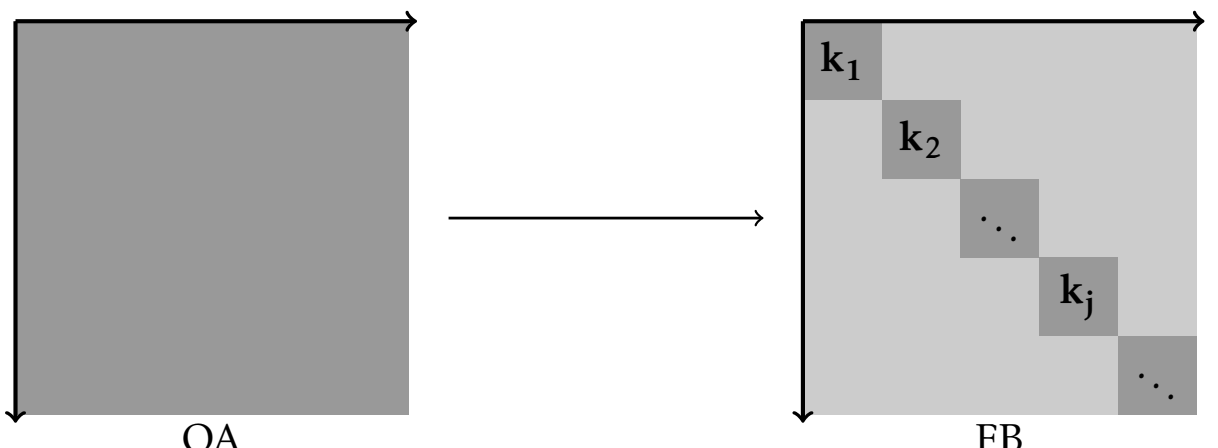

Figura 4.2: La matriz hamiltoniana de un sistema cristalino en términos de una base de Orbitales Atómicos (izquierda) y en términos de una base de Funciones de Bloch (derecha). Las flechas en los bordes resaltan el tamaño infinito de ambas matrices; las zonas más oscuras indican las zonas con elementos en general distintos de cero. Los bloques asociados a un punto $\mathbf{k}$ se encuentran etiquetados con éste.

\subsection{Construcción de Orbitales Cristalinos}

Si el Hamiltoniano que se utiliza es un Hamiltoniano monoelectrónico, como el de HF o el de KS, las soluciones de la ecuación 4.3 son llamadas Orbitales Cris- 
talinos (OC), $\psi_{i}(\mathbf{r} ; \mathbf{k}):[33]$

$$
\hat{H}^{\text {mono }} \psi_{i}(\mathbf{r} ; \mathbf{k})=E_{i}(\mathbf{k}) \psi_{i}(\mathbf{r} ; \mathbf{k}),
$$

donde $i$ indica el electrón al que esta asociado el orbital.

Los OC son construidos a su vez a partir de un conjunto de funciones de Bloch (FB) $\phi_{\mu}(\mathbf{r} ; \mathbf{k})$ :

$$
\psi_{i}(\mathbf{r} ; \mathbf{k})=\sum_{\mu} c_{\mu i}(\mathbf{k}) \phi_{\mu}(\mathbf{r} ; \mathbf{k}),
$$

de tal forma que los OC cumplen, al igual que las FB, el teorema de Bloch.

Una posible elección para la construcción de las FB es utilizar una Combinación Lineal de Orbitales Atómicos (LCAO), $\chi_{\mu}(\mathbf{r})$ :

$$
\phi_{\mu}(\mathbf{r} ; \mathbf{k})=\frac{1}{\sqrt{N}} \sum_{\mathbf{g}} \chi_{\mu}^{\mathbf{g}}\left(\mathbf{r}-\mathbf{R}_{\mu}\right) e^{i \mathbf{k} \cdot \mathbf{g}},
$$

con $R_{\mu}$ el sitio en el que se encuentra centrado el OA. El superíndice en la FB indica en que celda se ubica la función: $\chi_{\mu}\left(\mathbf{r}-\mathbf{R}_{\mu}-\mathbf{g}\right)=\chi_{\mu}^{\mathrm{g}}\left(\mathbf{r}-\mathbf{R}_{\mu}\right)$.

\subsubsection{El programa CRYSTAL}

El código utilizado en el presente trabajo de investigación, llamado CRYSTAL, se basa en la LCAO ya mencionada para la construcción de las FB. CRYSTAL es desarrollado principalmente por investigadores de la Universidad de Turín, en Italia, y el Laboratorio Daresbury de Reino Unido. Este programa está disponible desde 1988 [34] y tiene un desarrollo activo, siendo liberada la versión más reciente en 2017.[35]

En CRYSTAL la resolución de las integrales involucradas se realiza escribiendo los OA como una contracción de Funciones Tipo Gaussianas Esféricas Reales, 
$\gamma_{\mu j}^{\mathbf{g}}:[36]$

$$
\begin{gathered}
\gamma_{\mu}^{\mathbf{g}}(\mathbf{r})=N X_{\ell m}\left(\mathbf{r}-\mathbf{R}_{\mu}-\mathbf{g}\right) e^{-\beta\left(\mathbf{r}-\mathbf{R}_{\mu}-\mathbf{g}\right)^{2}}, \\
\chi_{\mu}^{\mathbf{g}}(\mathbf{r})=\sum_{j}^{n_{G}} d_{\mu j} \gamma_{\mu j}^{\mathbf{g}}\left(\alpha_{j} ; \mathbf{r}-\mathbf{R}_{\mu}\right),
\end{gathered}
$$

donde $X_{\ell m}$ es un Armónico Esférico Real y $n_{G}$ es el número de funciones utilizadas en la contracción.

\subsection{Construcción de las matrices}

Cuando se sustituye en la ecuación 4.4 la combinación lineal de FB que forman los $\mathrm{OC}$, se obtiene la siguiente relación matricial:

$$
\begin{array}{r}
\mathbf{H}(\mathbf{k}) \mathbf{C}(\mathbf{k})=\mathbf{S}(\mathbf{k}) \mathbf{C}(\mathbf{k}) \mathbf{E}(\mathbf{k}), \\
\mathbf{C}(\mathbf{k}) \mathbf{S}(\mathbf{k}) \mathbf{C}^{\dagger}(\mathbf{k})=\mathbf{I},
\end{array}
$$

con $\mathbf{S}(\mathbf{k})$ la matriz de traslape y $\mathbf{C}(\mathbf{k})$ la matriz de vectores propios. Mediante estas relaciones es posible calcular los coeficientes $c_{\mu i}(\mathbf{k})$ del las $\mathrm{FB}$, resolviendo las ecuaciones matriciales para cada vector $\mathbf{k}$ de la malla en el espacio recíproco.

En CRYSTAL estas matrices son inicialmente resueltas en el espacio real utilizando OA y después convertidas al espacio recíproco, repitiendo este procedimiento tantas veces como puntos $\mathbf{k}$ dentro de la primera zona de Brouillon se utilicen en el cálculo.[36] ${ }^{1}$ Por ejemplo, en el caso de un elemento de la matriz de traslape en el espacio recíproco, $S_{\mu \nu}(\mathbf{k})$, es posible mediante la ecuación 4.6

\footnotetext{
${ }^{1}$ Hasta dónde se indique lo contrario, la información sobre la integrales bielectrónicas viene de esta referencia.
} 
establecer la relación con su análogo en espacio real:

$$
\begin{aligned}
S_{\mu v}(\mathbf{k}) & =\left\langle\phi_{\mu}(\mathbf{k}) \mid \phi_{v}(\mathbf{k})\right\rangle \\
& =\int_{D} d \mathbf{r} \phi_{\mu}^{*}(\mathbf{r} ; \mathbf{k}) \phi_{\nu}(\mathbf{r} ; \mathbf{k}) \\
& =\frac{1}{\sqrt{N}} \sum_{\mathbf{g}} \sum_{\mathbf{g}^{\prime}} e^{-i \mathbf{k} \cdot \mathbf{g}} e^{i \mathbf{k} \cdot \mathbf{g}^{\prime}} \int_{D} d \mathbf{r} \chi_{\mu}^{*}(\mathbf{r}-\mathbf{g}) \chi_{\nu}\left(\mathbf{r}-\mathbf{g}^{\prime}\right) .
\end{aligned}
$$

Usando $\mathbf{r}-\mathbf{g}=\mathbf{r}^{\prime}$ y $\mathbf{g}-\mathbf{g}^{\prime}=-\mathbf{1}$ :

$$
\begin{aligned}
S_{\mu \nu}(\mathbf{k}) & =\frac{1}{N} \sum_{\mathbf{g}} \sum_{\mathbf{g}^{\prime}} e^{-i \mathbf{k} \cdot \mathbf{g}} e^{i \mathbf{k} \cdot \mathbf{g}^{\prime}} \int_{D} d \mathbf{r}^{\prime} \chi_{\mu}^{*}\left(\mathbf{r}^{\prime}\right) \chi_{v}\left(\mathbf{r}^{\prime}+\mathbf{g}-\mathbf{g}^{\prime}\right) \\
& =\frac{1}{N} \sum_{\mathbf{g}} \sum_{\mathbf{l}} e^{-i \mathbf{k} \cdot \mathbf{g}} e^{i \mathbf{k} \cdot \mathbf{g}+\mathbf{l})} \int_{D} d \mathbf{r}^{\prime} \chi_{\mu}^{*}\left(\mathbf{r}^{\prime}\right) \chi_{v}\left(\mathbf{r}^{\prime}-\mathbf{1}\right) \\
& =\frac{1}{N} \sum_{\mathbf{g}} e^{-i(\mathbf{k}-\mathbf{k}) \cdot \mathbf{g}} \sum_{\mathbf{1}} e^{i \mathbf{k} \cdot \mathbf{l}} \int_{D} d \mathbf{r}^{\prime} \chi_{\mu}^{*}\left(\mathbf{r}^{\prime}\right) \chi_{v}\left(\mathbf{r}^{\prime}-\mathbf{l}\right) \\
& =\sum_{\mathbf{1}} e^{i \mathbf{k} \cdot \mathbf{l}}<\chi_{\mu}^{0} \mid \chi_{v}^{1}>=\sum_{1} e^{i \mathbf{k} \cdot \mathbf{l}} S_{\mu v}^{l},
\end{aligned}
$$

donde $S_{\mu \nu}^{l}$ es un elemento de la matriz de traslape en el espacio real. Por tanto, si se calculan los elementos $S_{\mu \nu}^{l}$ en el espacio real es posible con esta relación obtener los elementos $S_{\mu \nu}(\mathbf{k})$ en el espacio recíproco para distintos puntos $\mathbf{k}$.

Una característica del sistema periódico es que la simetría traslacional que posee es transferible a las integrales presentes. Por ejemplo, para la integral de traslape se tiene:

$$
\left\langle\chi_{\mu}^{1} \mid \chi_{v}^{\mathbf{g}}\right\rangle=\left\langle\chi_{\mu}^{0} \mid \chi_{v}^{\mathbf{g}-1}\right\rangle=\left\langle\chi_{\mu}^{0} \mid \chi_{v}^{\mathbf{g}^{\prime}}\right\rangle
$$

donde $\mathbf{1}, \mathbf{g}$ y $\mathbf{g}^{\prime}=\mathbf{g}-\mathbf{l}$ son vectores de la malla en el espacio real. Dada esta propiedad siempre es posible situar una de las funciones en el origen $\mathbf{0}$. 


\subsubsection{La Matriz densidad}

La matriz densidad en el espacio recíproco, $\mathbf{P}(\mathbf{k})$, se define como:

$$
P_{\mu v}(\mathbf{k})=\sum_{n}^{N o c c .} c_{\mu n}^{*}(\mathbf{k}) c_{\nu n}(\mathbf{k})
$$

donde $n$ es el índice de los estados, y los $c_{j n}(\mathbf{k})$ son los coeficientes de la combinación lineal en la ecuación 4.5. Nocc. es el número de estados ocupados, y depende de $\mathbf{k}$. Mediante la transformación adecuada es posible obtener de esta expresión la matriz densidad en el espacio real $\left(P_{\mu v}^{\mathbf{g}}\right)$ :

$$
P_{\mu \nu}^{\mathbf{g}}=\sum_{n} \frac{1}{V_{B Z}} \int_{B Z} d \mathbf{k} e^{i \mathbf{k} \cdot \mathbf{g}} C_{\mu n}^{*}(\mathbf{k}) c_{\nu n}(\mathbf{k}) \theta\left(E_{F}-E_{n}(\mathbf{k})\right),
$$

donde $V_{B Z}$ es el volumen de la primera zona de Brillouin, $E_{F}$ es la energía de Fermi, $E_{n}$ la energía del $n$-ésimo estado, y $\theta\left(E_{F}-E_{n}(\mathbf{k})\right.$ es la función de Heaviside. La función de Heaviside es cero para estados virtuales, por lo que la suma sobre $n$ puede ir sobre todos los estados.

\subsubsection{La Matriz de Fock}

Si utilizamos el método HF, el Hamiltoniano a utilizar será el operador de Fock, $\hat{F}$, el cual puede descomponerse en cuatro contribuciones:

$$
\hat{F}=\hat{T}+\hat{Z}+\hat{C}+\hat{X},
$$

con $\hat{T}$ el operador de energía cinética, $\hat{Z}$ el de energía nuclear, $\hat{C}$ el de la interacción Coulómbica bielectrónica, y $\hat{X}$ el del intercambio de HF . Los elementos de matriz asociados a este operador en el espacio real tienen la forma:

$$
F_{\mu \nu}^{\mathbf{g}}=\left\langle\chi_{\mu}^{0}|\hat{F}| \chi_{\nu}^{\mathbf{g}}\right\rangle .
$$


Haciendo uso de (4.15), cada elemento de la matriz de Fock puede descomponerse en diferentes contribuciones:

- La integral cinética $T_{\mu \nu}^{\mathrm{g}}$ :

$$
T_{\mu \nu}^{\mathbf{g}}=-\frac{1}{2}\left\langle\chi_{\mu}^{0}\left|\nabla^{2}\right| \chi_{\nu}^{\mathbf{g}}\right\rangle=-\frac{1}{2} \int_{D} d \mathbf{r} \chi_{\mu}(\mathbf{r}) \nabla^{2} \chi_{v}(\mathbf{r}-\mathbf{g}),
$$

donde $D$ indica que la integral se realiza sobre el espacio real.

- La integral de interacción nuclear monoelectrónica $Z_{\mu v}^{g}$ :

$$
\begin{aligned}
Z_{\mu v}^{\mathbf{g}} & =-\sum_{\mathbf{h}} \sum_{A}^{N_{A}} \iint_{D} d \mathbf{r}_{1} d \mathbf{r}_{2} \chi_{\mu}^{0} \chi_{v}^{\mathbf{g}} \frac{1}{\left|\mathbf{r}_{1}-\mathbf{r}_{2}\right|} Z_{A} \delta\left(\mathbf{r}_{2}-\mathbf{R}_{\mathbf{A}}-\mathbf{h}\right) \\
& =-\sum_{\mathbf{h}} \sum_{A}^{N_{A}} Z_{A}\left\langle\chi_{\mu}^{0}\left|\frac{1}{\left|\mathbf{r}-\mathbf{R}_{\mathbf{A}}-\mathbf{h}\right|}\right| \chi_{v}^{\mathbf{g}}\right\rangle \\
& =-\sum_{\mathbf{h}} \sum_{A}^{N_{A}} Z_{A} \int_{D} d \mathbf{r} \chi_{\mu}(\mathbf{r}) \frac{1}{\left|\mathbf{r}-\mathbf{R}_{\mathbf{A}}-\mathbf{h}\right|} \chi_{v}(\mathbf{r}-\mathbf{g}),
\end{aligned}
$$

donde $\mathbf{r}_{1}$ es la posición asociada al electrón y $\mathbf{r}_{2}$ la posición del núcleo, el índice $A$ va sobre los núcleos, y $\mathbf{R}_{\mathbf{A}}$ es la posición de estos. El vector $\mathbf{h}$ es un vector de la malla en el espacio real; la suma va sobre todos los puntos de la malla, y por tanto es una suma infinita.

- La integral Coulómbica bielectrónica $C_{\mu \nu}^{g}$ :

$$
\begin{aligned}
C_{\mu \nu}^{\mathbf{g}} & =\sum_{\sigma, \omega} \sum_{\mathbf{g}^{\prime}} P_{\sigma \omega}^{\mathbf{g}^{\prime}} \sum_{\mathbf{h}}\left\langle\chi_{\mu}^{0} \chi_{\nu}^{\mathbf{g}}\left|\frac{1}{\left|\mathbf{r}_{1}-\mathbf{r}_{2}\right|}\right| \chi_{\sigma}^{\mathbf{h}} \chi_{\omega}^{\mathbf{h}+\mathbf{g}^{\prime}}\right\rangle \\
& =\sum_{\sigma, \omega} \sum_{\mathbf{g}^{\prime}} P_{\sigma \omega}^{\mathbf{g}^{\prime}} \sum_{\mathbf{h}} \iint_{D} d \mathbf{r}_{1} d \mathbf{r}_{2} \chi_{\mu}\left(\mathbf{r}_{1}\right) \chi_{v}\left(\mathbf{r}_{1}-\mathbf{g}\right) \frac{1}{\left|\mathbf{r}_{1}-\mathbf{r}_{2}\right|} \chi_{\sigma}\left(\mathbf{r}_{2}-\mathbf{h}\right) \chi_{\omega}\left(\mathbf{r}_{2}-\mathbf{h}-\mathbf{g}^{\prime}\right),
\end{aligned}
$$

donde $\mathbf{r}_{\mathbf{i}}$ es la coordenada del electrón $i$. Por las tres sumas involucradas, para el segundo electrón deben considerarse todos los OA utilizados en el cálculo. 
- La integral de intercambio HF $X_{\mu \nu}^{g}$ :

$$
\begin{aligned}
X_{\mu \nu}^{\mathbf{g}} & =-\frac{1}{2} \sum_{\sigma, \omega} \sum_{\mathbf{g}^{\prime}} P_{\sigma \omega}^{\mathbf{g}^{\prime}} \sum_{\mathbf{h}}\left\langle\chi_{\mu}^{0} \chi_{\sigma}^{\mathbf{h}}\left|\frac{1}{\left|\mathbf{r}_{1}-\mathbf{r}_{2}\right|}\right| \chi_{\nu}^{\mathbf{g}} \chi_{\omega}^{\mathbf{h}+\mathbf{g}^{\prime}}\right\rangle \\
& =-\frac{1}{2} \sum_{\sigma, \omega} \sum_{\mathbf{g}^{\prime}} P_{\sigma \omega}^{\mathbf{g}^{\prime}} \sum_{\mathbf{h}} \iint_{D} d \mathbf{r}_{1} d \mathbf{r}_{2} \chi_{\mu}\left(\mathbf{r}_{1}\right) \chi_{\sigma}\left(\mathbf{r}_{\mathbf{1}}-\mathbf{h}\right) \frac{1}{\left|\mathbf{r}_{1}-\mathbf{r}_{2}\right|} \chi_{\nu}\left(\mathbf{r}_{2}-\mathbf{g}\right) \chi_{\omega}\left(\mathbf{r}_{2}-\mathbf{h}-\mathbf{g}^{\prime}\right) .
\end{aligned}
$$

De estas contribuciones se reconocen como las más costosas computacionalmente a la integral Coulómbica bielectrónica y la integral de intercambio HF, siendo comúnmente agrupadas como integrales bielectrónicas. ${ }^{2}$ Dada su importancia en el funcionamiento práctico del programa, y para los fines de este trabajo, en adelante se ahondará solamente en el cálculo de las contribuciones asociadas a estas integrales.

El operador hamiltoniano electrostático de $\mathrm{HF}, \hat{H}^{H F}$, tiene la siguiente forma:

$$
\hat{H}^{H F}=\frac{1}{2}(\hat{F}+\hat{Z}+\hat{T}) .
$$

La energía total por celda:

$$
E_{t o t}=\mathrm{N}+\sum_{\mu, v, \mathbf{g}} P_{\mu \nu}^{\mathbf{g}} H_{\mu \nu}^{\mathbf{g}}=\mathrm{N}+\sum_{\mu, v, \mathbf{g}} P_{\mu \nu}^{\mathbf{g}}\left(F_{\mu v}^{\mathbf{g}}+Z_{\mu v}^{\mathbf{g}}+T_{\mu v}^{\mathbf{g}}\right)
$$

con $\mathrm{N}$ el término de energía potencial internuclear.

Si desarrollamos el término de Fock:

$$
E_{t o t}=\mathrm{N}+\sum_{\mu, v, \mathbf{g}} P_{\mu \nu}^{\mathbf{g}}\left(C_{\mu v}^{\mathbf{g}}+X_{\mu v}^{\mathbf{g}}+2 Z_{\mu \nu}^{\mathbf{g}}+2 T_{\mu \nu}^{\mathbf{g}}\right)
$$

\footnotetext{
${ }^{2}$ En el caso del método de KS se calculan en general las integrales Coulómbicas bielectrónicas, y si se utiliza un funcional $E_{X C}$ híbrido deben calcularse también las integrales de intercambio.
} 
de donde podemos agrupar distintas contribuciones a la energía:

$$
E_{\text {tot }}=E_{N u c l e a r}+E_{K}+E_{N-e}+E_{C}+E X .
$$

En especifico, los términos Coulómbico y de Intercambio tiene la siguiente forma:

$$
\begin{gathered}
E_{C}=\frac{1}{2} \sum_{\mu \nu} \sum_{\mathbf{g}} P_{\mu \nu}^{\mathbf{g}} \sum_{\sigma \omega} \sum_{\mathbf{g}^{\prime}} P_{\sigma \omega}^{\mathbf{g}^{\prime}} \sum_{\mathbf{h}}\langle\underbrace{\chi_{\mu}^{\mathbf{0}} \chi_{v}^{\mathbf{g}}}_{T_{1}} \overbrace{\frac{1}{\left|\mathbf{r}_{\mathbf{1}}-\mathbf{r}_{2}\right|}|\underbrace{\chi_{\sigma}^{\mathbf{h}} \chi_{\omega}^{\mathbf{h}+\mathbf{g}^{\prime}}}_{T_{1}}\rangle}^{T_{2}}, \\
E_{X}=-\frac{1}{4} \sum_{\mu \nu} \sum_{\mathbf{g}} \underbrace{P_{\mu \nu}^{\mathbf{g}}}_{T_{4}} \sum_{\sigma \omega} \sum_{\mathbf{g}^{\prime}} \underbrace{P_{\sigma \omega}^{\mathbf{g}^{\prime}}}_{T_{5}} \sum_{\mathbf{h}}\langle\underbrace{\chi_{\mu}^{\mathbf{0}} \chi_{\sigma}^{\mathbf{h}}}_{T_{3}} \overbrace{\frac{1}{\left|\mathbf{r}_{1}-\mathbf{r}_{2}\right|}\left|\chi_{\nu}^{\mathbf{g}} \chi_{\omega}^{\mathbf{h}+\mathbf{g}^{\prime}}\right\rangle}^{T_{3}} .
\end{gathered}
$$

Los términos señalados con llaves están asociados al truncado de las sumas infinitas, discutidas en la siguiente sección.

\subsection{Tratamiento de las sumas infinitas}

En las ecuaciones previas existen sumas infinitas que requieren ser truncadas para poder ser resueltas computacionalmente. A continuación se muestran detalles de este procedimiento.

Los OA de un átomo pueden agruparse en capas $\lambda$, las cuales están caracterizadas por tener los mismos números cuánticos $n$ y $\ell$ (como las capas $1 s, 2 p$ ), o solo el mismo número cuántico $n$ (como la capa $s p$ ). A cada capa está asociada una gaussiana adjunta $\gamma_{\lambda}$; esta gaussiana es de tipo $s$, está normalizada y tiene exponente igual al menor de los exponentes de las funciones que componen dicha capa. Las 
gaussianas adjuntas, al ser funciones representativas de la máxima dispersión de las funciones que conforman una capa, resultan ser de utilidad para estimar si la interacción entre dos capas (de átomos distintos o de un mismo átomo) o entre dos átomos contribuirá de forma relevante en las distintas contribuciones energéticas.

En CRYSTAL las integrales en (4.17) hasta (4.20) son evaluadas bajo un criterio de traslape, utilizando las gaussianas adjuntas para estimar el traslape entre los OA asociados a un átomo o una capa. Por ejemplo, en el caso de un par de orbitales $d$, se tienen 25 integrales de traslape a evaluar. Antes de realizar todas estas integrales se evalúa el traslape entre las gaussianas adjuntas de ambos orbitales $d$ y con ello se decide si se calculará o no el conjunto de las 25 integrales. De igual forma puede evaluarse el traslape entre las gaussianas adjuntas más dispersas de dos átomos, y mediante el resultado tomar decisiones respecto a las integrales entre los OA de ambos átomos.

Este criterio de traslape limita el conjunto infinito de puntos de la malla involucrados en las integrales a un conjunto finito: si el traslape es menor a un valor $10^{-T_{i}}$, donde $T_{i}$ establece esta tolerancia, se truncan las sumas infinitas o se realiza alguna aproximación.

En el caso de la integral bielectrónica $\left\langle\mu^{0} \nu^{\mathbf{g}} \mid \sigma^{\mathbf{h}} \omega^{\mathbf{h}+\mathbf{g}^{\prime}}\right\rangle$, esta tenderá a cero si:

- $\mathbf{h}$ es muy grande,

- $\nu^{\mathbf{g}}$ está lejos de $\mu^{0}$ o $\omega^{\mathbf{g}^{\prime}}$ está lejos de $\sigma^{0}$ (g o $\mathbf{g}^{\prime}$ muy grandes),

- Los exponentes de las gaussianas son muy grandes.

Con base en este comportamiento en el código se utilizan 5 tolerancias basadas en el traslape de las gaussianas adjuntas, las cuales se describen a continuación, puntualizando su efecto en las integrales bielectrónicas: 
$T_{1}$ - El truncado sobre la suma en $\mathbf{g}$ o $\mathbf{g}^{\prime}$ ocurre si el traslape entre las gaussianas adjuntas asociadas a $\left\langle\mu^{0} \mid v^{\mathbf{g}}\right\rangle \mathrm{o}\left\langle\sigma^{\mathbf{h}} \mid \omega^{\mathbf{h}+\mathbf{g}^{\prime}}\right\rangle$, respectivamente, es menor a $10^{-T_{1}}$.

$T_{2}$ - La integral Coulómbica es evaluada de forma exacta si las distribuciones de carga están traslapadas, de lo contrario se resuelve por una expansión multipolar, bajo la aproximación de que el potencial electrostático generado por una distribución de carga en un punto externo a esta puede ser aproximado por una expansión multipolar de la distribución.

Si combinamos las contribuciones de interacción núcleo-electrón (4.18) y electrón-electrón (4.19), sin integrar (4.18) sobre la coordenada asociada al núcleo, es posible reordenar términos de la siguiente forma:

$$
\begin{aligned}
C_{12}^{\mathbf{g}}+Z_{12}^{\mathbf{g}} & =\sum_{\sigma, \omega} \sum_{\mathbf{g}^{\prime}} P_{\sigma \omega}^{\mathbf{g}^{\prime}} \sum_{\mathbf{h}} \iint_{D} d \mathbf{r}_{1} d \mathbf{r}_{2} \chi_{\mu}\left(\mathbf{r}_{1}\right) \chi_{v}\left(\mathbf{r}_{1}-\mathbf{g}\right) \frac{1}{\left|\mathbf{r}_{1}-\mathbf{r}_{2}\right|} \chi_{\sigma}\left(\mathbf{r}_{2}-\mathbf{h}\right) \chi_{\omega}\left(\mathbf{r}_{2}-\mathbf{h}-\mathbf{g}^{\prime}\right) \\
& -\sum_{\mathbf{h}} \sum_{A}^{N_{A}} \iint_{D} d \mathbf{r}_{1} d \mathbf{r}_{2} \chi_{\mu}^{0} \chi_{v}^{\mathbf{g}} \frac{1}{\left|\mathbf{r}_{1}-\mathbf{r}_{2}\right|} Z_{A} \delta\left(\mathbf{r}_{2}-\mathbf{R}_{\mathbf{A}}-\mathbf{h}\right) \\
& =\sum_{\mathbf{h}} \sum_{A}^{N_{A}} \iint_{D} d \mathbf{r}_{1} d \mathbf{r}_{2} \chi_{\mu}\left(\mathbf{r}_{1}\right) \chi_{v}\left(\mathbf{r}_{1}-\mathbf{g}\right) \frac{1}{\left|\mathbf{r}_{1}-\mathbf{r}_{2}\right|} \\
& \left(\sum_{\sigma \in A} \sum_{\omega} \sum_{\mathbf{g}^{\prime}} P_{\sigma \omega}^{\mathbf{g}^{\prime}} \chi_{\sigma}\left(\mathbf{r}_{2}-\mathbf{h}\right) \chi_{\omega}\left(\mathbf{r}_{2}-\mathbf{h}-\mathbf{g}^{\prime}\right)-Z_{A} \delta\left(\mathbf{r}_{2}-\mathbf{R}_{\mathbf{A}}-\mathbf{h}\right)\right) .
\end{aligned}
$$

El término entre paréntesis en la ecuación 4.27 puede asociarse a una pseudocarga total $\rho_{A}$ del átomo o capa $A$, análoga a la expresión de carga de Mulliken en sistemas moleculares:

$$
\rho_{A}\left(\mathbf{r}-\mathbf{R}_{\mathbf{A}}\right)=\sum_{\sigma \in A} \sum_{\omega} \sum_{\mathbf{g}^{\prime}} P_{\sigma \omega}^{\mathbf{g}^{\prime}} \chi_{\sigma}(\mathbf{r}) \chi_{\omega}\left(\mathbf{r}-\mathbf{g}^{\prime}\right)-Z_{A} \delta\left(\mathbf{r}-\mathbf{R}_{\mathbf{A}}\right) .
$$

Es posible mediante (4.28) expresar la distribución de carga de la celda como:

$$
\rho(\mathbf{r})=\sum_{A} \sum_{\mathbf{h}} \rho_{A}\left(\mathbf{r}-\mathbf{R}_{\mathbf{A}}-\mathbf{h}\right) .
$$


Usando (4.27) y (4.28) obtenemos una expresión para la interacción Coulómbica entre la distribución de carga de un electrón y la pseudo-carga de una capa o átomo:

$$
C_{\mu \nu}^{\mathbf{g}}+Z_{12}^{\mathbf{g}}=\sum_{A} \sum_{\mathbf{h}} \iint_{D} d \mathbf{r}_{1} d \mathbf{r}_{2} \rho_{\mu \nu}\left(\mathbf{r}_{\mathbf{1}}-\mathbf{g}\right) \frac{1}{\left|\mathbf{r}_{\mathbf{1}}-\mathbf{r}_{2}\right|} \rho_{A}\left(\mathbf{r}_{2}-\mathbf{R}_{\mathbf{A}}-\mathbf{h}\right),
$$

donde $\rho_{\mu \nu}\left(\mathbf{r}_{\mathbf{1}}-\mathbf{g}\right)=\chi_{\sigma}(\mathbf{r}) \chi_{\omega}\left(\mathbf{r}-\mathbf{g}^{\prime}\right)$. En la ecuación 4.30 la suma sobre $\mathbf{h}$ está asociada a la distancia entre las dos pseudocargas interactuantes. Los vectores $\mathbf{h}$ se dividen, bajo el criterio de traslape, en dos subconjuntos:

- El conjunto finito $\{H\}$ en el cual las distribuciones se traslapan y las integrales se evalúan de forma exacta.

- El conjunto infinito $\left\{H_{A}\right\}$ donde las integrales son aproximadas realizando una expansión multipolar de $\rho_{A}$ y son evaluadas de forma analítica hasta infinito.

Si el traslape entre las distribuciones de carga $\rho_{A}^{h}$ y $\rho_{\mu \nu}, S_{\mu \nu, A}$, es menor a cierto valor entonces el vector asociado $\mathbf{h}$ es asignado al conjunto $\left\{H_{A}\right\}$. Para esto se evalúa el traslape entre $\mu \nu$ y la gaussiana adjunta de la capa de $\sigma^{\mathbf{h}}, \mathrm{y}$ se evalúa si es menor a $10^{-T_{2}}$.

Si las distribuciones de carga no traslapan para ningún $\mathbf{h}$ entonces dichos elementos de la matriz de Coulomb son expresados en términos de los momentos multipolares de $\rho_{A} \gamma_{\ell m}(A)$ y de integrales de campo $\varphi_{\ell m \mu v}\left(\mathbf{g}, \mathbf{R}_{\mathbf{A}}+\mathbf{h}\right)$ :

$$
\begin{gathered}
\gamma_{\ell m}(A)=\int_{D} d \mathbf{r}_{2} \rho_{A}\left(\mathbf{r}_{2}-\mathbf{R}_{\mathbf{A}}\right) N_{\ell m} \mathbf{X}_{\ell m}\left(\mathbf{r}_{2}-\mathbf{R}_{\mathbf{A}}\right), \\
\varphi_{\ell m \mu \nu}\left(\mathbf{g}, \mathbf{R}_{\mathbf{A}}+\mathbf{h}\right)=\int_{D} d \mathbf{r}_{\mathbf{1}} \chi_{\mu}\left(\mathbf{r}_{\mathbf{1}}\right) \chi_{\nu}\left(\mathbf{r}_{2}-\mathbf{g}\right) \mathbf{X}_{\ell m}\left(\mathbf{r}_{\mathbf{1}}-\mathbf{R}_{\mathbf{A}}-\mathbf{h}\right)\left(\frac{1}{\left|\mathbf{r}_{\mathbf{1}}-\mathbf{R}_{\mathbf{A}}-\mathbf{h}\right|}\right)^{2 \ell+1}, \\
C_{\mu \nu}^{\mathbf{g}}=\sum_{A} \sum_{\ell m}\left(\sum_{\mathbf{h}} \gamma_{\ell m}(A) \varphi_{\ell m \mu \nu}\left(\mathbf{g}, \mathbf{R}_{\mathbf{A}}+\mathbf{h}\right)\right) .
\end{gathered}
$$


La suma infinita sobre $\mathbf{h}$ puede realizarse utilizando la técnica de Ewald, con lo cual se reduce a un número finito de términos.

$T_{3}$ - De forma a equivalente al caso de $T_{1}$, pero para las integrales de intercambio (4.20), se evalúa si el traslape entre las gaussianas adjuntas asociadas a $\left\langle\mu^{0} \mid \sigma^{\mathbf{h}}\right\rangle$ o $\left\langle v^{\mathbf{g}} \mid \omega^{\mathbf{h}+\mathbf{g}^{\prime}}\right\rangle$ es menor a $10^{-T_{3}}$. Si alguno de ambos no cumple este requisito la suma sobre $\mathbf{h}$ en estas integrales es truncada.

$T_{4}, T_{5}$ - En las integrales de intercambio la suma sobre $\mathbf{g}^{\prime}$ es truncada si $\left\langle\mu^{0} \mid v^{\mathbf{g}}\right\rangle<$ $10^{-T_{4}}$, y la suma $\mathbf{g}^{\prime}$ es truncada si $\left\langle\sigma^{\mathbf{h}} \mid \omega^{\mathbf{h}+\mathbf{g}^{\prime}}\right\rangle<10^{-T_{5}}$; como en este criterio las integrales de traslape van sobre funciones en distintos centros a estas tolerancias se les denota como de pseudotraslape. La justificación de este criterio está en el decaimiento exponencial de los elementos de la matriz densidad con la distancia entre los dos centros.

Adicionalmente, del conjunto $\{H\}$ obtenido bajo la tolerancia $T_{2}$, algunas de las integrales pueden ser aproximadas por una expansión bipolar de las dos distribuciones de carga $\mu \nu$ y $\sigma \omega$, cuando la distribución de carga del segundo electrón es externa a la distribución de carga del primero. El valor de traslape que controla cuando se realiza esta expansión se encuentra definido dentro del código, y se trata por separado de las tolerancias antes descritas. La expansión bipolar implica que ambas distribuciones de carga sean escritas como expansiones multipolares.

\subsubsection{Estrella de vectores}

En el proceso de truncar las integrales infinitas es de gran utilidad considerar la simetría del sistema a estudiar, evitando así el cálculo de varias integrales equivalentes. Un concepto que se aprovecha en CRYSTAL es el de estrella de vectores, un conjunto de vectores obtenido por la aplicación de las operaciones de simetría del grupo puntual del cristal sobre un vector.[37] Dicho conjunto de vectores parte 
de un mismo orígen y al ser producto de las operaciones de simetría del grupo espacial tienen el mismo módulo (Figura 4.3).

Las estrellas de vectores son auxiliares en la selección de los vectores $\mathbf{g}$ y $\mathbf{g}^{\prime}$ para la evaluación de las integrales bielectrónicas, permitiendo una clasificación de la gran cantidad de vectores posibles.

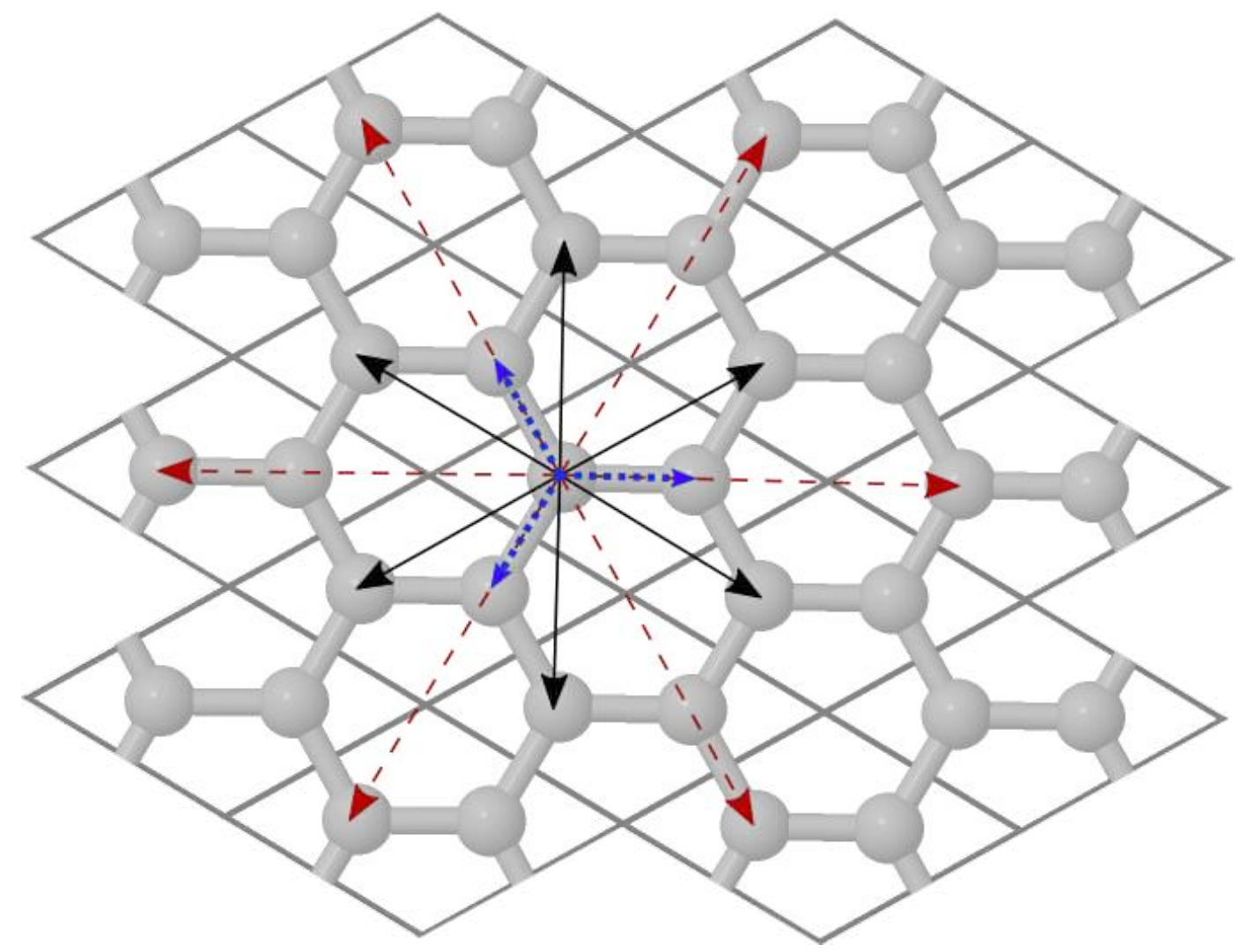

Figura 4.3: Tres estrellas de vectores en grafeno partiendo de un mismo centro atómico. Los vectores de una estrella comparten el mismo diseño en la imagen.

\subsubsection{Evaluación de las integrales bielectrónicas}

Una vez que hemos truncado las sumas infinitas, podemos evaluar de forma explícita las integrales que nos restan en el conjunto $\{H\}$, y el resto de integrales del conjunto $\left\{H_{A}\right\}$ mediante la expansión multipolar y la técnica de Ewald. Para cada vector $\mathbf{h}$ en $\{H\}$, y las combinaciones con los vectores $\mathbf{g}$ y $\mathbf{g}^{\prime}$ restantes tras el 
truncado correspondiente, hay que resolver la siguiente integral:

$$
C_{\mu \nu}^{\mathbf{g}, \mathbf{g}^{\prime}, \mathbf{h}}=\iint_{D} d \mathbf{r}_{1} d \mathbf{r}_{2} \chi_{\mu}^{0} \chi_{\nu}^{\mathbf{g}} \frac{1}{\left|\mathbf{r}_{1}-\mathbf{r}_{2}\right|} \chi_{\sigma}^{\mathbf{h}} \chi_{\omega}^{\mathbf{h}+\mathbf{g}^{\prime}}
$$

En el caso de las integrales de intercambio análogamente tenemos:

$$
X_{\mu \nu}^{\mathbf{g}, \mathbf{g}^{\prime}, \mathbf{h}}=\iint_{D} d \mathbf{r}_{1} d \mathbf{r}_{2} \chi_{\mu}^{0} \chi_{\sigma}^{\mathbf{h}} \frac{1}{\left|\mathbf{r}_{1}-\mathbf{r}_{2}\right|} \chi_{\nu}^{\mathbf{g}} \chi_{\omega}^{\mathbf{h}+\mathbf{g}^{\prime}}
$$

Estas integrales son idénticas a las encontradas en sistemas moleculares, por lo que es posible resolverlas por las mismas técnicas; en el código se utiliza el método de McMurchie-Davidson [38] no sólo para resolver las integrales bielectrónicas de forma exacta, sino además para resolver las integrales involucradas en la aproximación por expansión multipolar en el conjunto $\left\{H_{A}\right\}$. Además, si la integral sólo involucra capas $s$ o $s p$, se puede utilizar una rutina adaptada del programa Gaussian70 que resulta más eficiente.

\subsection{Algoritmo del código}

Dada la complejidad de resolver las ecuaciones descritas de una forma eficiente y precisa, la implementación del código de CRYSTAL ha requerido del desarrollo inteligente de diversos algoritmos. En esta sección se describe el esquema general del programa, enfocando la descripción en el camino que lleva a la resolución de las integrales bielectrónicas.[36] 


\subsubsection{Programa principal}

El código principal llama a tres rutinas básicas: una que da inicio al programa e inicializa variables necesarias para el paralelismo por $\mathrm{MPI}^{3}$, otra que se encarga de la ejecución principal y una última que da por finalizado el programa y el entorno MPI.

La segunda de estas rutinas coordina las funciones básicas del programa, leyendo inicialmente el archivo de entrada que contiene la información mínima para la ejecución (tipo de sistema, grupo espacial, tipo de átomos, base, nivel de cálculo). Una vez leída esta información, se ejecutan diversas rutinas según el tipo de cálculo requerido; en el caso de que el cálculo sea de tipo single point se prepara la información básica sobre las integrales a realizar, y después se ejecuta una rutina de campo autoconsistente, que realiza la técnica del mismo nombre.

\subsubsection{Método de campo autoconsistente}

El método de Campo Auto-Consistente (SCF) efectuado en dicha rutina sigue, a grandes rasgos, el siguiente esquema:

1. Se propone una matriz densidad inicial dado el conjunto base de OA y la malla de puntos k en la zona de Brillouin. Dicha malla se construye bajo el esquema de Monkhorst-Pack.

2. Se evalúan los elementos $F_{\mu \nu}^{\mathrm{g}}$ en el espacio real y posteriormente se transforman al espacio recíproco.

3. Se diagonaliza para cada punto $\mathbf{k}$ el bloque correspondiente de la matriz de Fock y se determina la matriz de coeficientes (ecuación 4.9).

\footnotetext{
${ }^{3}$ Interfaz de Paso de Mensajes (MPI) se refiere a un estándar utilizado principalmente para el paralelismo entre distintos equipos mediante memoria distribuida.
} 
4. Se calcula el nivel de Fermi, y con esto se obtiene una nueva matriz densidad en el espacio recíproco. Se transforma esta matriz al espacio real.

5. Se calcula el cambio en la energía entre el ciclo presente y el anterior.

6. Si el cambio en la energía es mayor a cierta tolerancia, se repite el procedimiento desde el paso 2. Si el cambio es menor, se termina con el ciclo.

Para la contribución bielectrónica a la matriz de Fock en el paso 2 se utiliza una rutina específica, descrita a continuación.

\subsubsection{Selección y evaluación de las integrales bielectrónicas}

La rutina que coordina la evaluación de las integrales bielectrónicas se encarga de seleccionar las integrales a evaluar, de llamar a las rutinas que las calculan, y de sumar y almacenar sus resultados en un solo bloque, que luego es multiplicado por el bloque correspondiente de la matriz densidad. Cada elemento de este bloque corresponde a las contribuciones $F_{\mu v-b i e l}^{g}$,

$$
F_{\mu \nu-b i e l}^{g}=I_{C}+I_{X}=\sum_{\sigma, \omega, \mathbf{g}^{\prime}}\left\{\sum_{\mathbf{h}}<\mu^{0} \nu^{\mathbf{g}}\left|\sigma^{\mathbf{h}} \omega^{\mathbf{h}+\mathbf{g}^{\prime}}>-\frac{1}{2} \sum_{\mathbf{h}}<\mu^{0} \sigma^{\mathbf{h}}\right| \nu^{\mathbf{g}} \omega^{\mathbf{h}+\mathbf{g}^{\prime}}>\right\} P_{\sigma \omega}^{\mathbf{g}} .
$$

La rutina consiste en siete ciclos anidados que seleccionan los conjuntos de parámetros para los cuales las integrales deben ser resueltas de forma explícita o por alguna aproximación multipolar (Figura 4.4). En estos ciclos se realiza el truncado de las sumas infinitas asociadas a las integrales bielectrónicas; la selección de los vectores $\mathbf{g}$ y $\mathbf{g}^{\prime}$ con este fin se realiza de forma explícita, mientras que para el truncado sobre $\mathbf{h}$ se llama a una rutina específica. En orden del cíclo más externo al más interno, los elementos sobre los que se itera son los siguientes:

1. Los pares $\chi_{\mu} \chi_{\nu}$. 
2. Las estrellas de vectores $\mathbf{g}$.

3. Los vectores irreducibles $\mathbf{g}$ de la estrella.

4. Los pares $\chi_{\sigma} \chi_{\omega}$.

5. Pares $\chi_{\sigma} \chi_{\omega}$ conectados por simetría.

6. Las estrellas de vectores $\mathbf{g}^{\prime}$

7. Los vectores $\mathbf{g}^{\prime}$ de la estrella.

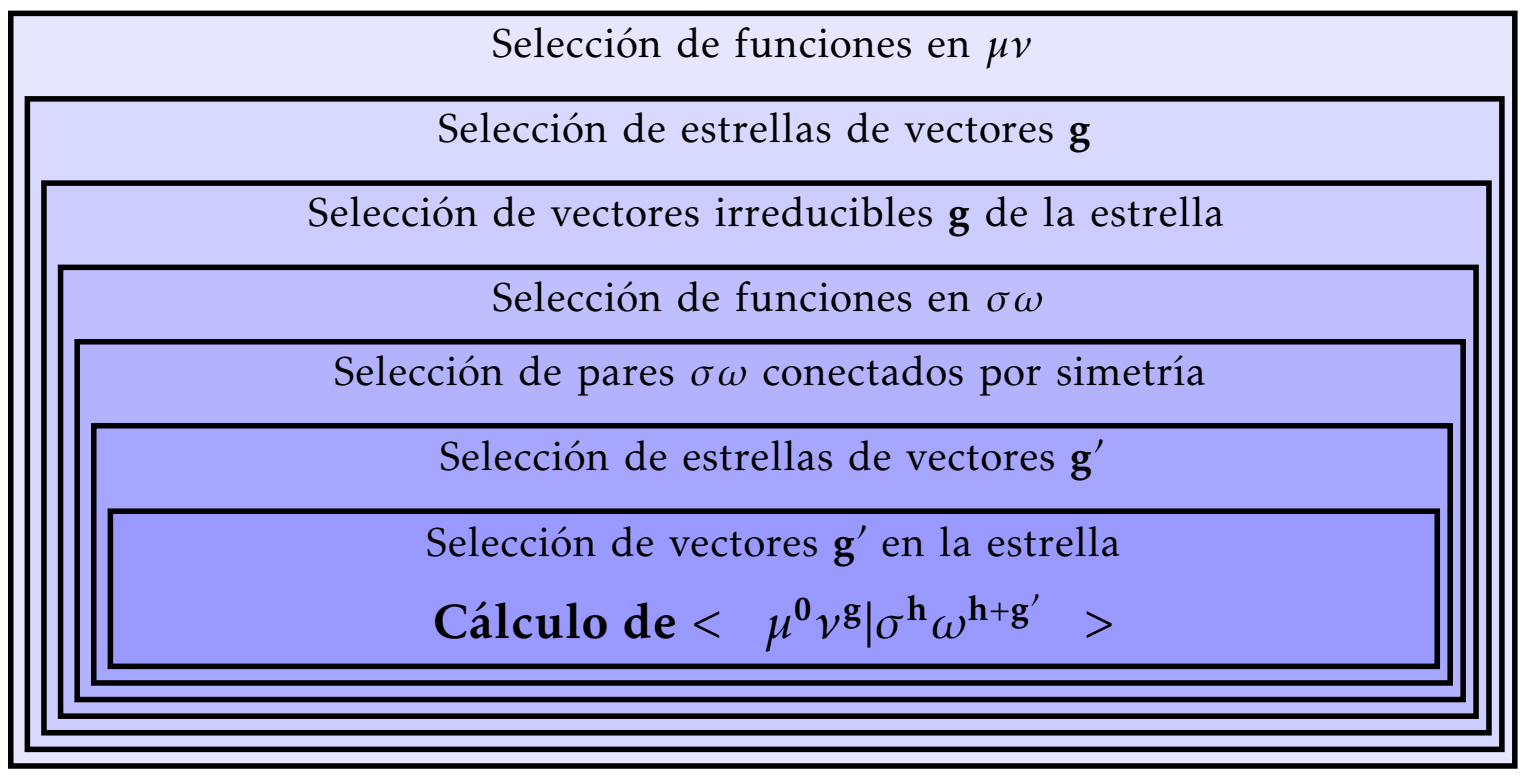

Figura 4.4: Diagrama de la rutina de evaluación de integrales bielectrónicas. Cada recuadro representa un cíclo encargado de la selección de los elementos de la integral.

Cada que se recolectan suficientes parámetros para el cálculo de alguna integral se llama a la rutina correspondiente, sea el método de McMurchie-Davidson o el método adaptado de Gaussian70. Los resultados obtenidos son sumados como se indica en la ecuación 4.36, con lo cual se construye una matriz, posteriormente multiplicada por el bloque correspondiente de la matriz densidad, para finalmente obtener el bloque asociado de la matriz de Fock. 


\section{Densidad de los cíclos del algorítmo}

Con el fin de estimar en que región del cálculo de las integrales bielectrónicas se encuentra el mayor esfuerzo computacional se colocaron contadores que muestran la cantidad de iteraciones asociada a cada uno de los ciclos anidados. En la Tabla 4.1 se muestran resultados obtenidos para el $\mathrm{SiO}_{2}$ con estructura tipo rutilo, para una supercelda con ocho celdas unitarias de este mismo sistema, para el $\mathrm{ZrO}_{2}$ con estructura monoclínica, y para una supercelda de $\mathrm{MnO}$ con estructura cúbica.

\begin{tabular}{|c|c|c|c|c|}
\hline Átomos & $2 \mathrm{Si}, 4 \mathrm{O}$ & $4 \mathrm{Zr}, 8 \mathrm{O}$ & $8 \mathrm{Mn}, 8 \mathrm{O}$ & $16 \mathrm{Si}, 32 \mathrm{O}$ \\
\hline Tamaño de la Base & 132 & 232 & 320 & 1,024 \\
\hline \hline Ciclo 1 & 129 & 413 & 1,517 & 2,467 \\
Ciclo 2 & 238 & 998 & 1,652 & 2,607 \\
Ciclo 3 & 263 & 1,128 & 2,572 & 2,789 \\
Ciclo 4 & 40,765 & 527,904 & $6,008,192$ & $12,740,152$ \\
Ciclo 5 & 157,780 & $2,104,644$ & $8,189,352$ & $47,290,870$ \\
Ciclo 6 & 523,215 & $12,302,411$ & $13,770,556$ & $47,015,284$ \\
Ciclo 7 & $1,450,710$ & $14,249,966$ & $43,726,496$ & $54,951,748$ \\
\hline
\end{tabular}

Tabla 4.1: Iteraciones por ciclo para una iteración del método SCF. Las primeras dos columnas corresponden a celdas unitarias de $\mathrm{SiO}_{2}$ y $\mathrm{ZrO}_{2}$, mientras que la tercera y cuarta corresponden a superceldas de $\mathrm{MnO}$ y $\mathrm{SiO}_{2}$.

En todos estos sistemas se observa un gran aumento en el número de iteraciones entre el ciclo 3 y el 4 , mientras en el resto de los casos las razones entre ciclos son mucho menores, como puede observarse en Figura 4.5. Claramente, por cada iteración sobre el ciclo 3 hay una gran cantidad de iteraciones sobre el ciclo 4, y consecuentemente sobre los ciclos 5, 6 y 7. Respecto al número de integrales totales se observa que el número de integrales en estos sistemas relativamente sencillos se encuentran en el orden de millones

Esta información resulta de gran importancia si se desea optimizar el cálculo de las integrales bielectrónicas mediante el cálculo paralelizado de las mismas, permitiendo diseñar en qué forma puede ser posible u óptima la distribución del cálculo sobre múltiples procesadores. Cabe señalar que los ciclos contenidos dentro 
del tercero son los que actualmente se ejecutan en paralelo en el ćodigo utilizando CPUs en la versión del código que disponemos.

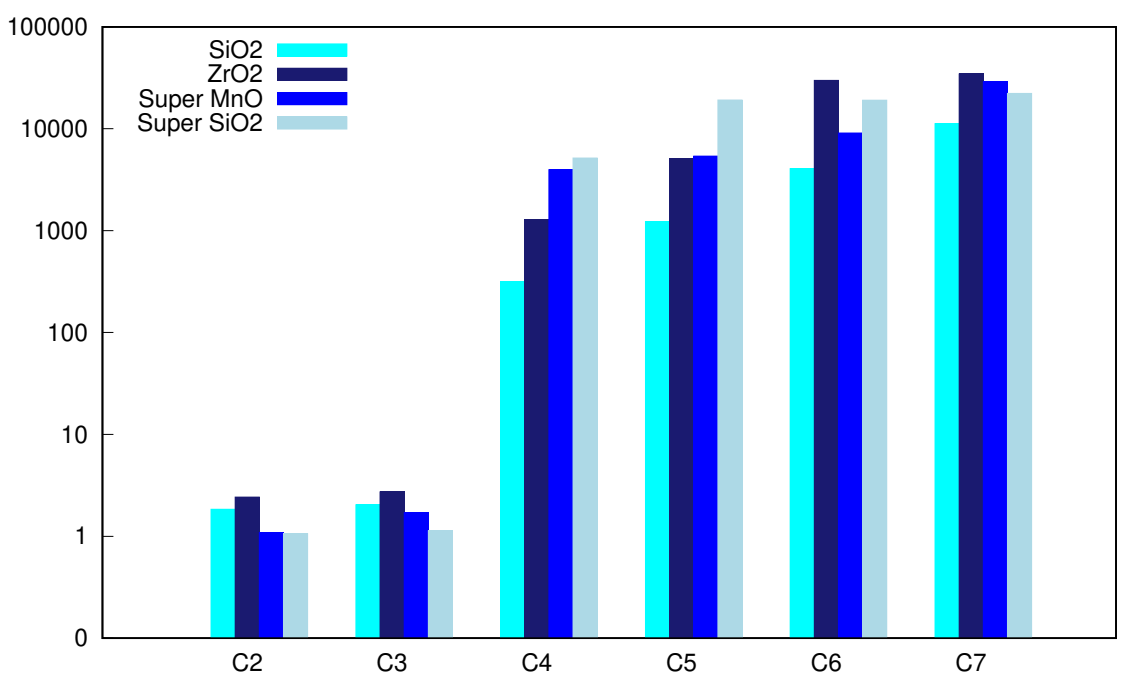

Figura 4.5: Número de iteraciones por ciclo respecto al Ciclo 1. Ci representa el Ciclo $i$.

\subsection{Cómputo heterogéneo}

Es evidente que el desarrollo de la química computacional no sólo depende del desarrollo de la teoría, sino además del estado del arte en computación y equipos de cómputo de la época. Tal como se menciona en el Capítulo 1 los métodos más utilizados en los últimos años son los de HF y KS, ya que aunque los métodos Post-HF permiten cálculos más exactos el poder de cómputo que requieren sigue siendo un reto para la tecnología actual. En décadas previas resultaba complejo el estudio de sistemas de cientos de átomos aún mediante HF, por lo cual hubo un auge en el desarrollo de métodos semi-empíricos. En un futuro, si el cómputo cuántico se desarrolla a un nivel suficiente, puede que los métodos Post-HF se conviertan en los más utilizados, pero con ello probablemente será necesario el desarrollo de técnicas adaptadas a la forma en que estas computadoras cuánticas se presenten.[39] 
Un factor de alta relevancia en nuestra época es la inmensa variedad de equipos de cómputo que podemos encontrar. Tenemos dispositivos de tamaño y objetivos diversos, en una lista larga que incluye teléfonos inteligentes, laptops, computadoras de escritorio y servidores dedicados. Para cada uno de estos dispositivos hay además una gran variedad de opciones disponibles para cada componente que incluyen, ya sea procesadores, tarjetas de video o de red, o unidades de almacenamiento. Con el ritmo de desarrollo tecnológico actual es esperable que la variedad de dispositivos aumente, lo cual afecta ya en las técnicas de desarrollo de código y los métodos de integración de los distintos equipos para su uso unificado.

Es en este escenario que nace el cómputo heterogéneo, esquema en el cual los distintos dispositivos de cómputo utilizados para una tarea tienen distintas capacidades o formas de ejecución.[40] Esta iniciativa, presente desde hace ya algunos años, resulta relevante en la química computacional, a parecer del autor, por dos motivos principales:

- Llama a desarrollar programas para una mayor variedad de dispositivos, permitiendo a un mayor público el uso del código y evitando la dependencia de un sólo tipo de equipo o proveedor.

- Abre la posibilidad de aprovechar los beneficios de cada dispositivo, con la posibilidad de acelerar cálculos y disminuir costos económicos y energéticos.

En esta sección se hará mención de la variedad de dispositivos actual en cuanto al procesamiento de datos y algunas herramientas importantes en el ámbito heterogéneo. 


\subsubsection{Dispositivos de procesamiento actuales}

\section{CPU}

Si bien predomina los de la empresa Intel en servidores y equipos personales, la variedad de CPUs existentes es cada vez más evidente en distintos entornos. Una muestra de esto está en la lista TOP500, en la cual se muestran las supercomputadoras con mayor capacidad de cómputo del mundo.[41] En el listado más reciente al momento los tres primeros lugares no utilizan en su infraestructura base procesadores Intel, encontrándose en cambio procesadores Power de IBM en los dos primeros lugares y procesadores Sunway de fabricación expresa para el supercomputador en el tercero.

Aún cuando existen varias coincidencias en el diseño de muchos de los CPUs actuales, las diferencias técnicas existentes impiden muchas veces que un programa ejecutado en alguno de ellos pueda correr utilizando otro. Incluso en el caso en el que esto es logrado, en muchas ocasiones las diferencias en los tiempos de ejecución e incluso numéricas pueden ser importantes.

\section{GPU}

Para poder aprovechar correctamente el potencial que brindan las GPUs (Unidad de Procesamiento Gráfico, por sus siglas en inglés) para acelerar un código, es importante comprender, al menos esquemáticamente, cómo se ejecuta una tarea dentro de estos dispositivos.[42] En esta sección en donde resulte conveniente se usará el término dispositivo para identificar a la GPU y el término huésped para la computadora donde se encuentra la GPU.

La característica principal de una GPU son las miles de unidades de procesamiento que posee. Aunque individualmente estas unidades son generalmente 
más lentas que las de un CPU, el tiempo empleado para realizar miles de operaciones en paralelo sobre una GPU puede resultar menor en algunos casos que el empleado en la ejecución con CPU. Dado que el potencial de la GPU reside en la cantidad de operaciones que puede realizar al mismo tiempo, las tareas donde es apreciable la disminución en el tiempo de cómputo son aquellas que constan de cantidades enormes de operaciones equivalentes.

Un detalle de gran importancia a considerar para la ejecución sobre el dispositivo es que éste no tiene acceso directo a la memoria del huésped, por lo que es necesario transferir datos del huésped al dispositivo y viceversa. Esta transferencia de información resulta ser el paso limitante en los procedimientos que se realizan en el dispositivo, por lo cual debe utilizarse al mínimo.

Los GPUs son una de las principales razones por las cuales el cómputo heterogéneo ha adquirido su popularidad actual, demostrando en distintos escenarios disminuciones importantes tanto en tiempo de ejecución como en consumo energético. Una prueba de ello es de nuevo la lista Top500, donde al menos 6 de los 10 primeros lugares, incluyendo al primer y segundo lugar, utilizan GPUs. Al igual que con el CPU existe un proveedor predominante de estos dispositivos, la empresa NVIDIA, pero la presencia de otros GPUs, como los de AMD o los de Intel, cada vez es más notoria. De nuevo de forma análoga al CPU, actualmente resulta complejo poder correr un programa aprovechando eficientemente GPUs de distintos proveedores.

\section{Otros dispositivos y procesadores}

Existen otros dispositivos para el procesamiento de datos cuya relevancia actual merece ser notada. Sin dar muchos detalles técnicos, se listan los siguientes:

- Los coprocesadores Xeon Phi de Intel son un punto intermedio entre las capacidades del CPU y la cantidad de operaciones que manejan un GPU.[43] El 
sexto lugar del Top500 hace uso expreso de estos dispositivos.

- Los procesadores tipo ARM, utilizados en teléfonos inteligentes y otros dispositivos móviles, que destacan principalmente por poder ser empleados en equipos de bolsillo.

- Algunos dispositivos diseñados expresamente para inteligencia artificial y análisis de datos, como los AP y los chips neuromórficos.[40]

\subsubsection{Algunas herramientas para cómputo heterogéneo}

\section{Compiladores PGI}

Como se mencionó para los CPUs y GPUs, un problema básico para implementar cómputo heterogéneo con una aplicación es poder ejecutar ésta en distintos dispositivos eficientemente. Una de las complicaciones suele ser que para cada dispositivo exista un solo compilador que genere ejecutables funcionales. Por dicha razón algunos códigos generan distintas versiones de un programa para distintas plataformas, pero el tiempo de desarrollo adicional que esto significa disuade a varios equipos de desarrollo de realizar esta tarea.

En este contexto, los compiladores de la empresa PGI son una opción a considerar para la construcción de aplicaciones multiplataforma.[44] Estos compiladores permiten construir aplicaciones para su ejecución en procesadores Intel, AMD y Power IBM utilizando un mismo código fuente, evitando así un gasto mayor en tiempo de desarrollo. Además, incluyen varias instrucciones que permiten acelerar la ejecución según el tipo de procesador que se utilice, con lo cual pueden alcanzarse desempeños similares e inclusive mejores a los obtenidos con los compiladores nativos del procesador. Una de las características más notables es que permite además aprovechar esquemas de paralelismo populares, admitiendo OpenMP para el paralelismo por CPU, y CUDA y OpenACC para GPU. 
Si bien los beneficios de utilizar estos compiladores son notables, para algunas opciones de aceleramiento hay restricciones respecto a los errores de sintaxis que pueden ignorarse o interpretarse en un código, lo cual puede implicar modificaciones mayores en programas que son compilados de forma directa en otros compiladores, como los de GNU o los de Intel. Por ello, si se desea utilizar los compiladores de PGI extensivamente es recomendable su uso desde etapas iniciales del desarrollo del código, aunque sigue siendo viable adaptar programas ya establecidos.

\section{OpenACC}

OpenACC es un modelo de programación ideado para permitir la ejecución de un algoritmo en distintos aceleradores utilizando un solo código, donde el acelerador puede ser un procesador multicore, un GPU o un coprocesador como los Xeon Phi. Esto es posible gracias al compilador, que interpreta directivas añadidas al código y produce un programa ejecutable en la plataforma necesitada.[45, 46] Actualmente existen códigos de estructura electrónica que incorporan el uso de OpenACC para acelerar su ejecución. $[47,48]$

El enfoque de directivas ${ }^{4}$ de OpenACC disminuye los tiempos de desarrollo necesarios para poder ejecutar secciones de código en paralelo usando CPUs o GPUs, y de forma frecuente el estándar es actualizado para permitir su uso en más esquemas de programación y más dispositivos. Los compiladores de PGI se mantienen en constante sincronía con los avances de OpenACC, siendo una de las mejores opciones si se piensa utilizar esta opción.

\footnotetext{
${ }^{4}$ Para más información sobre este esquema ver Apéndice A
} 


\subsection{CRYSTAL sobre cómputo heterogéneo}

Una parte importante del trabajo realizado en esta tesis consistió en probar algunas de las ideas del cómputo heterogéneo en el código CRYSTAL, en su versión de 2014.[49] En esta sección se hablará de dichos esfuerzos, así como las perspectivas al respecto.

\subsubsection{Pruebas realizadas}

\section{Uso de GPU para evaluar integrales bielectrónicas}

Puesto que la cantidad de integrales bielectrónicas a evaluar de algunos sistemas de interés puede estar en el orden de millones (ver Tabla 4.1), la evaluación de estas integrales de forma eficiente con la tecnología actual debe de ser en paralelo. Algunos códigos de estructura electrónica para sistemas finitos ya aprovechan GPUs para la evaluación de estas integrales, con disminuciones de los tiempos de ejecución importantes. $[48,50]$ Resulta por lo tanto atractiva la posibilidad de aprovechar el uso de estos dispositivos para resolver estas ecuaciones en CRYSTAL.

De la información disponible sobre OpenACC se espera que el costo por su uso sea bajo en cuanto a la cantidad de modificaciones necesarias para ejecutar el código en GPU, por lo que para este trabajo se seleccionó esta herramienta para la adaptación del código. Para la compilación se utilizaron los compiladores de PGI 18.4, Community Edition, por lo que la versión de OpenACC usada fue la 2.6.

De lo observado en la Tabla 4.1 se tomó la decisión de adaptar el código correspondiente a los ciclos 4 al 7 de la rutina de las integrales bielectrónicas (Figura 4.4), dada la gran cantidad de evaluaciones que esta parte involucra y siguiendo el esquema de paralelización por MPI que el código ya tiene. Otra razón por la cual se tomó esta decisión fue la presencia de funciones que escriben en la memoria del 
huésped en los ciclos previos, lo cual representa un problema para su ejecución usando el GPU, siendo necesarias costosas transferencias de información entre el dispositivo y el huésped, o dado el caso modificaciones mayores al código.

Durante el tiempo que se intentaron implementar las directivas en la región de código deseada aparecieron distintas complicaciones, que pueden ser atribuidas a dos razones principales: la antigüedad del código que se encarga de resolver las integrales bielectrónicas, y el enfoque estrictamente serial que tiene el código. A continuación se describen los procedimientos realizados y las dificultades encontradas.

En un primer paso se intentó usar una directiva directa para la paralelización del cíclo 4, y consecuentemente los cíclos 5 al 7. Esta aproximación no permitió la compilación del programa, por lo que con el apoyo de los mensajes del compilador y la documentación disponible de OpenACC se avanzó en la compilación de código funcional. El principal problema que se detectó con esta iniciativa se detalla en los siguientes párrafos.

Dada la antigüedad del código, es común encontrar funciones de Fortran que actualmente están en desuso y son desaconsejadas por ser factores que propician código poco claro. El uso de Common Blocks, al permitir renombramiento de variables y cambiar los intervalos e incluso la dimensionalidad de los arreglos contenidos de función a función, se desaconseja desde la aparición de los Módulos, que no sufren de estas complicaciones. Hasta la versión más reciente de OpenACC no existe una herramienta que permita la trasferencia de variables globales ubicadas en Common Blocks al dispositivo.

El primer problema que se encontró durante la compilación del código con las directivas de OpenACC fue la presencia de Common Blocks en la rutina principal y en las rutinas que ésta llama. La solución por la que se optó fue la transferencia de estas variables a Módulos. Esta conversión resulta costosa en cuanto a lectura de código, ya que es necesario rastrear en qué rutinas, dentro y fuera de la región 
a paralelizar, estas variables son definidas, modificadas o utilizadas.

Esta tarea puede ser facilitada utilizando herramientas visuales como debuggers, pero incluso en estas herramientas el uso de Common Blocks no está bien soportado, siendo necesaria la lectura de los archivos del código fuente con la ayuda de herramientas de la terminal de Linux para el filtrado de la información.

Un problema serio que se encontró dentro de los Common del código fue el desajuste de los arreglos de función a función. La solución en este caso involucró la creación de nuevos arreglos globales que contuvieran algunos de los arreglos desfasados, y por tanto fue necesario cambiar por completo las variables utilizadas en las rutinas involucradas. En general los Common que nos interesan no fueron completamente sustituidos del código, sino solamente donde resultaran relevantes para el procedimiento SCF. Una versión completamente funcional del código requeriría atender este problema.

Como se mencionó previamente, OpenACC admite el uso de las variables globales contenidas dentro de Módulos, pero requiere de la directiva declare para este fin. Utilizando los mensajes del compilador y el código fuente fue posible filtrar qué variables dentro de los Módulos ya existentes eran necesarias para la ejecución en GPU, ya que el uso de todas las variables de todos los Módulos involucrados implicaría el movimiento de grandes cantidades de datos entre el huésped y el dispositivo.

Otros errores encontrados estaban asociados al uso de GoTo, una herramienta de Fortran que permite indicar donde debe continuar la ejecución del código utilizando etiquetas numéricas. Esta herramienta también es actualmente desaconsejada, ya que propicia ejecuciones difíciles de seguir. Si bien OpenACC admite la presencia de estas instrucciones, una de las estructuras producidas por el uso de GoTo en una rutina no pudo ser correctamente interpretada por el compilador, enviando errores que no revelaban la naturaleza del problema. Al respecto es probable que esto se deba a la complejidad del esquema de GoTo utilizado, resultado en 
una lectura de código complicada. En este caso fue necesario pensar en una nueva forma de ejecutar dicha estructura, siendo la solución actual convertir dicha sección del algoritmo en una rutina separada, con un nuevo Módulo asociado y algunos pequeños cambios en la lógica de los GoTo contenidos.

Con la solución de estos problemas fue posible compilar una versión ejecutable del código, pero dicha ejecución llevó a resultados y comportamientos erróneos debido principalmente al enfoque serial del programa original. Dada la complejidad de analizar los problemas de ejecución encontrados en la amplia región delimitada con kernels, se tomó como segundo enfoque implementar directivas de OpenACC en el ciclo 7 hasta que la ejecución fuera posible y los resultados correctos, para después pasar gradualmente a los ciclos externos hasta llegar al cuarto.

Uno de los problemas es la aparición de variables cuyo valor depende de un ciclo previo. Por ejemplo:

$$
\begin{aligned}
& \text { Do } i=1, N \\
& a=a+1 \\
& \text { rutina }(a) \\
& \text { Enddo }
\end{aligned}
$$

En este caso, la variable $a$ en el ciclo 1 será $a_{0}+1$, con $a_{0}$ el valor de $a$ antes del Do, y en el ciclo 2 será también $a_{0}+1$, pero como la ejecución de los ciclos ocurre originalmente de forma serial para este ciclo $a=\left(a_{0}+1\right)+1=a_{0}+2$. Si añadimos la directiva kernels (ver Apéndice A): 


$$
\begin{aligned}
& \text { ! } \$ \text { acc kernels } \\
& \text { Do } i=1, N \\
& a=a+1 \\
& \text { rutina }(a) \\
& \text { Enddo } \\
& \text { ! \$acc end kernels }
\end{aligned}
$$

Es importante notar que cada iteración del Do será un hilo de ejecución en el GPU, y que no existe un control sobre el orden en el que éstos se ejecutan. Por lo tanto, puede ocurrir que $a=a_{0}+1$ para todos los casos, con lo cual todos ejecutarán rutina con este argumento y claramente los resultados serán distintos al del programa serial. En este caso el compilador detecta correctamente este problema, por lo que genera código de ejecución serial para evitar resultados incorrectos.

Según sea el caso, se utilizaron dos posibles aproximaciones para solucionar el error. En el caso de que a no sea nuevamente utilizado en la región, es posible indicar que debe ocurrir una reducción, utilizando la opción reduce $(+: a)$. Con esta opción cada hilo tendrá un valor de $a$, en este caso $1, \mathrm{y}$ al final de la región los valores de $a$ en cada hilo serán sumados a $a_{0}$, con lo cual el resultado final será equivalente al del proceso serial.

En el caso del ejemplo mostrado, donde $a$ es utilizado posteriormente, no es posible esto ya que es importante que cada hilo tenga un valor determinado para que el llamado a rutina sea el correcto. En este caso es necesario modificar el algoritmo: 


$$
\begin{aligned}
& \text { ! } \$ \text { acc kernels } \\
& \text { Do } \mathrm{i}=1, \mathrm{~N} \\
& \mathrm{a}=\mathrm{a}+\mathrm{i} \\
& \text { rutina }(\mathrm{a}) \\
& \mathrm{b}[\mathrm{i}]=\mathrm{a} \\
& \text { Enddo } \\
& ! \$ \mathrm{acc} \text { end kernels } \\
& \mathrm{a}=\mathrm{b}[\mathrm{N}]
\end{aligned}
$$

El valor de $a$ está asociado al ciclo $i$, el cual puede ser utilizado como un referente para identificar el hilo de ejecución, ya que a cada hilo corresponde un valor de $i$. Así, cada hilo llamará con el argumento correcto a rutina, y la ejecución producirá resultados correctos. Si el último valor de $a$ es requerido fuera de la región entonces es necesario almacenar los valores de $a$ de cada hilo en un arreglo cuya posición este conectada con el valor de $i$ del hilo. Si bien involucra un incremento en el uso de memoria, este método permite recuperar el valor de $a$ para cualquier valor de $i$, y es posible solamente trasferir este valor del dispositivo al huésped.

Dos problemas adicionales asociados al manejo de las variables de Módulos fueron detectados. La directiva declare puede reservar espacio en el dispositivo para la variable global (opción create) o copiar el valor que se le asigna dentro del Módulo, pero es necesario indicarle en qué parte del código el valor de dicha variable será igual al utilizado en el dispositivo inicialmente. Con este fin se utiliza la directiva update, acompañada de la variable que desea actualizarse y si se actualiza la región en el dispositivo o en el huésped. El rastreo de estas variables se realizó con las herramientas de la terminal de Linux y con un debugger; esta tarea demanda bastante tiempo dada la basta cantidad de variables globales involucradas en la región de interés.

El otro problema es el de los arreglos dinámicos contenidos en Módulos. El compilador es capaz de determinar el tamaño del arreglo en el dispositivo si en- 
cuentra una instrucción Alloc para el apartado de memoria. El problema es que el código no utiliza de forma explícita esta instrucción para asignarle tamaño a los arreglos, sino que usa una interfaz que posteriormente llama a Alloc y el compilador no identifica correctamente esta operación. En este caso es necesario cambiar los llamados a la interfaz por ejecuciones directas de Alloc, ya que no existe otra forma de que el compilador genere las instrucciones para generar el arreglo en el dispositivo.

Un detalle técnico a señalar es que, para verificar el funcionamiento del código, se modificaron y recortaron funciones del código en su versión serial. Con esto fue posible verificar el correcto funcionamiento de las fracciones de código que iban siendo adaptadas, por lo cual es posible asegurar que, hasta el punto alcanzado, dicha parte del programa funcionaba correctamente y sobre el GPU. El alcance final de estas modificaciones bajo este enfoque fue hasta la evaluación de las integrales usando la aproximación multipolar, restando por tanto la evaluación de las integrales por los métodos de McMurchie-Davidson y de Gaussian70.

Si se consideran los detalles mencionados, y esperando que la mayoría de los errores futuros fueran de naturaleza idéntica o similar, sería posible avanzar continuamente en la implementación de OpenACC en la región deseada de la evaluación de las integrales. Sin embargo, fue detectada una nueva problemática para seguir con este enfoque. El ciclo 7 involucra la presencia de múltiples arreglos en el dispositivo, ya sea de nueva creación o trasferidos desde el huésped. Durante las últimas ejecuciones de prueba del programa modificado, utilizando el $\mathrm{SiO}_{2}$ y una base de tamaño regular, la cantidad de memoria requerida por el GPU fue del orden de $500 \mathrm{MB}$. Esto implica que por cada iteración del ciclo 6 dicha cantidad de memoria sería necesaria en el GPU; si tomamos en cuenta la Tabla 4.1 la cantidad de memoria necesaria para escalar el uso de directivas hasta el ciclo 6 sería en promedio 3 veces más (tres iteraciones de C6 por una del ciclo 5), y si se alcanza el ciclo 4 en promedio serían 13 veces más, siendo esto alrededor de 6.5 GB. La memoria total del GPU usado en el servidor de pruebas es de 2GB; consideran- 
do que aún resta la sección de evaluación directa resultaría imposible aprovechar este GPU usando el enfoque utilizado. Aun si consideramos GPUs de última generación, con 12-32 GB de almacenamiento, el uso del programa en dichas condiciones sería altamente costoso siendo que este sistema es bastante sencillo en comparación con otros de mayor complejidad e interés tecnológico.

\section{Ejecución con procesadores POWER}

Los procesadores POWER, distribuidos por IBM,[51] resultan ser de gran interés en la actualidad ya que, como se menciona anteriormente, debido a sus capacidades son utilizados en las dos supercomputadoras más potentes de la actualidad. Un punto notable es el acceso abierto que existe a la arquitectura POWER, por medio de la iniciativa OpenPOWER, promoviendo así la colaboración con otros grupos y empresas.[52] Entre algunas de sus características más notorias están la gran cantidad de núcleos que pueden poseer, así como la cantidad de hilos que cada uno de estos núcleos puede ejecutar en paralelo.

En el caso de CRYSTAL no existe una versión del programa que pueda ejecutarse en servidores con procesadores POWER, estando limitado a su uso con procesadores Intel. En este sentido, fueron realizadas algunas pruebas de compilación y ejecución de CRYSTAL sobre un equipo con procesadores POWER9, la versión mas reciente de estos, para ver la viabilidad de ejecutar el programa en este tipo de servidores y cuales son las ventajas y desventajas de dicha ejecución.

Los datos de los procesadores POWER9 del equipo utilizado se destacan en la Tabla 4.2, así como los del procesador Intel del equipo con el que se comparó, la supercomputadora Yoltla. Para la compilación en el equipo POWER fueron utilizados dos compiladores: los compiladores PGI, de la versión 18.10, y los compiladores XLF de IBM, nativos para este tipo de equipos. En el caso del equipo Intel fueron utilizados por un lado los mismos compiladores PGI que en POWER, y por otro se usó el binario precompilado que comercialmente distribuye CRYSTAL. 


\begin{tabular}{cccccc}
\hline Equipo & Procesador & Sockets & Núcleos/socket & Hilos/núcleo & Frecuencia \\
\hline IBM & POWER9 & 2 & 20 & 4 & $2.4 \mathrm{GHz}$ \\
Intel & Xeon E5-2670v2 & 2 & 10 & 1 & $2.5 \mathrm{GHz}$ \\
\hline
\end{tabular}

Tabla 4.2: Especificaciones de los procesadores usados en las pruebas. Para comparar se usaron dos nodos Intel con el fin de equiparar al equipo IBM en núcleos.

Como se ha mencionado antes CRYSTAL puede ser ejecutado en paralelo usando MPI, por medio del ejecutable PCRYSTAL. Existe otro ejecutable, MPPCRYSTAL, que sigue un esquema de paralelismo MPI distinto a PCRYSTAL y está basado en sistemas de memoria distribuida. En el caso de las compilaciones con PGI fue utilizado OpenMPI, en la versión 2.1.2 que viene incluida con los compiladores. En cuanto a XLF fue utilizado Spectrum MPI, programa nativo para IBM. El binario precompilado se ejecutó con Intel MPI 5.1.3.

Los resultados de las pruebas realizadas se muestran en la Tabla 4.3. A continuación se detalla cual fue el procedimiento para obtenerlos.

\begin{tabular}{ccccc}
\hline \hline Procesos & Compilador & Equipo & Tiempo (s) & Observaciones \\
\hline \hline 80 & Binario & Yoltla & 1043.51 & Versión comercial \\
40 & Binario & Yoltla & 1465.30 & Versión comercial \\
80 & Intel & Yoltla & 1319.99 & \\
40 & Intel & Yoltla & 1940.62 & \\
80 & PGI & Yoltla & 1389.73 & \\
40 & PGI & Yoltla & 1963.89 & \\
20 & PGI & Power & 2438.70 & Con -enable-mpi-thread-multiple \\
40 & PGI & Power & 2308.81 & Con -enable-mpi-thread-multiple \\
80 & PGI & Power & 3925.04 & Con -enable-mpi-thread-multiple \\
160 & PGI & Power & 3078.70 & Con -enable-mpi-thread-multiple \\
40 & PGI & Power & 1672.84 & Sin opción para hilos \\
\hline 40 & IBM & Power & 1766.37 & Sin opción para hilos \\
40 & IBM & Power & 2613.37 & Con -use-hwthread-cpus \\
80 & IBM & Power & 2743.34 & Con -use-hwthread-cpus \\
80 & IBM & Power & 2083.83 & Con -oversubscribe \\
\hline
\end{tabular}

Tabla 4.3: Tiempos de ejecución del sistema $\mathrm{ZrO}_{2}$ usando CRYSTAL14 en distintos equipos.

La compilación del programa con PGI pudo realizarse de forma exitosa con solo una modificación en una línea del código. Lo que resultó ser complejo fue el uso 
de distintas opciones de ejecución MPI, dada la cantidad de procesos o hilos que cada equipo puede manejar. Los procesadores Intel de Yoltla tienen la capacidad de usar dos hilos por núcleo, pero tiene esta opción deshabilitada desde el BIOS, por lo que solamente puede usarse un hílo por núcleo. En el equipo POWER, por el contrario, tenía habilitada la opción para múltiples hilos por núcleo, por lo que era posible ejecutar hasta 120 unidades de procesamiento en el mismo servidor.

En la fase inicial de pruebas, dado que eran detectados los 120 procesos, se intentó utilizar al máximo los hilos del equipo POWER. Para poder realizar esto se incluyó la opción -enable-mpi-thread-multiple en la construcción de OpenMPI, ya que sin esta opción no era posible ejecutar más de 40 procesos. Con esta opción se obtuvieron resultados usando 40, 80 y 120 procesos. Como puede observarse en la Tabla 4.3, en todos estos casos los tiempos de ejecución fueron mucho mayores a los obtenidos con el equipo comparativo, por lo que buscamos otras opciones para la compilación y ejecución.

Buscando descartar problemas por OpenMPI o los compiladores PGI fue utilizado XLF y Spectrum MPI, dado que esta combinación de herramientas para desarrollo nativo de IBM debería resultar en un rendimiento mejorado. Para la ejecución con Spectrum MPI fue necesario añadir la opción -use-hwthread-cpus o la opción -oversubscribe para poder utilizar múltiples hilos; la segunda opción produjo los menores tiempos de ejecución. El tiempo obtenido usando 80 procesos y -oversubscribe resultó menor que el obtenido con PGI y OpenMPI, pero claramente siguen siendo peor que el obtenido con el equipo comparativo.

Luego de estos resultados y una mayor búsqueda de información sobre MPI se encontró la razón por la cual los tiempos en ambos casos fueron tan grandes. El estándar MPI puede ejecutar en paralelo múltiples hilos, pero para ello es necesario por un lado añadir algunas opciones para la ejecución, y por otro hacer uso de algunas instrucciones dentro del código.[53] En CRYSTAL no hay presencia de instrucciones internas para el uso de hilos por MPI, por lo que era esperable que la 
ejecución con muchos hilos no fuera óptima. En general, este tipo de paralelismo no se realiza eficientemente mediante MPI, para lo cual resulta una mejor opción OpenMP; en estos casos suele recomendarse una aproximación híbrida de OpenMP con MPI.[54]

Con conocimiento de esta situación se realizaron pruebas comparativas usando solamente un proceso por núcleo, sin incluir opciones en la compilación de MPI ni en la ejecución. Los resultados que se observan muestran que los tiempos de ejecución son ahora comparables con los obtenidos con el equipo comparativo. Los tiempos del binario fueron los mejores, lo cual puede atribuirse al uso de opciones de optimización en la compilación.

Estos resultados muestran dos puntos importantes. Primero, que el uso de un compilador flexible para múltiples plataformas, como el de PGI, permite construir a partir de un mismo código fuente ejecutables con desempeño similar. Segundo, que para poder aprovechar al máximo procesadores como los POWER que permiten la ejecución de múltiples hilos es necesario adaptar el código, ya sea usando MPI u OpenMP. Otra opción para hacer uso de los múltiples hilos es usar bibliotecas cuyas funciones estén diseñadas para ello, como algunas de álgebra lineal disponibles en la red.[55, 56]

\subsubsection{Perspectivas}

En cuanto al uso del GPU para la evaluación de las integrales bielectrónicas en CRYSTAL, si bien puede ser viable por lo observado en las pruebas, requiere de un rediseño en el algoritmo actualmente utilizado o en el esquema de almacenamiento de los datos utilizados, tal que los arreglos dentro el GPU sean de un tamaño razonable. Dicho desarrollo requiere de un esfuerzo de tiempo completo, ya que al ser este un código maduro las modificaciones en una parte fundamental del programa repercutirán en el resto de sus capacidades. Si bien aún mediante 
del uso de directivas de OpenACC parece ser una labor complicada, los beneficios que puede tener aprovechar el uso del GPU u otros aceleradores en la resolución de estas integrales o en otros procedimientos siguen siendo atractivos como para intentarlo.

Respecto al uso de CRYSTAL en equipos POWER se muestra que los compiladores PGI permiten una ejecución aceptable del código sobre esta plataforma, abriendo así la posibilidad de que sea ejecutado en infraestructuras de cómputo que las incluyan. Por otro lado, para el máximo aprovechamiento de este tipo de equipos sería necesario realizar algunas modificaciones al código para poder explotar el máximo de hilos posibles por procesador. Una alternativa es el uso de bibliotecas ya optimizadas para este tipo de ejecución, particularmente para el manejo de las grandes matrices que utiliza el código. Es importante notar que, en la última versión de CRYSTAL, el paso más costoso en sistemas grandes es la diagonalización de la matriz de Fock.[57] 

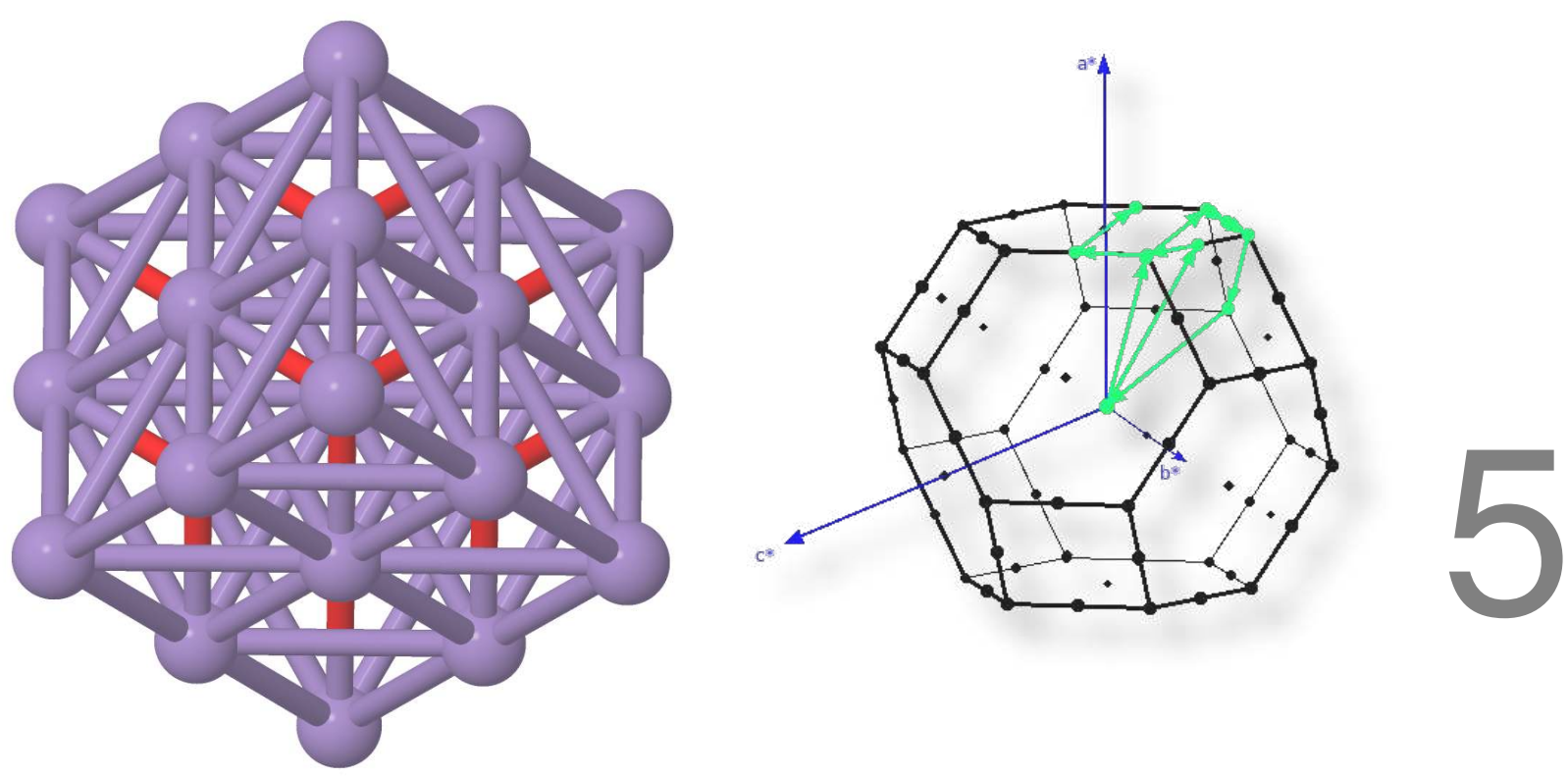

\section{Efecto del intercambio exacto}

\section{en algunos óxidos metálicos}

En este capítulo se discutirán los resultados obtenidos para los siguientes sistemas:

- Dos óxidos de metales de transición:

- Óxido de Manganeso (II) (MnO) en estructura sal de roca,

- Zirconia $\left(\mathrm{ZrO}_{2}\right)$ en estructura monoclínica,

- Tres óxidos de metaloides del grupo del carbono (grupo IVA) con estructura tipo rutilo:

- Stishovita $\left(\mathrm{SiO}_{2}\right)$,

- Argutita $\left(\mathrm{GeO}_{2}\right)$,

- Casiterita $\left(\mathrm{SnO}_{2}\right)$.

En el caso del $\mathrm{MnO}$, algunos de los resultados reportados en la literatura incluyen el funcional GGA PBE, los funcionales híbridos B3LYP y HSE03, y un fun- 
cional híbrido de intercambio apantallado basado en LDA.[58] En el caso del funcional PBE, el funcional híbrido HSE03 y el funcional híbrido basado en LDA el band gap resulta subestimado, con valores entre 0.8 y $3.2 \mathrm{eV}$ comparado con los valores experimentales que oscilan entre 3.8 y 4.2. Solamente B3LYP da un valor que entra en este rango, con $3.92 \mathrm{eV}$. Otros métodos, como LDA+U complementado con $\mathrm{G}_{0} \mathrm{~W}_{0}$, también arroja resultados más cercanos al intervalo experimental.[59]

En el caso del $\mathrm{ZrO}_{2}$, están reportados cálculos con funcionales $\mathrm{LDA}, \mathrm{LDA}+\mathrm{GW}_{0}$, PW1PW, PBE0 y B3LYP.[60] Respecto al valor experimental, entre 5.3 y $5.8 \mathrm{eV}$, PBE0 y B3LYP arrojan valores adecuados al intervalo, con 5.7 y 5.2 para gap indirecto y 6.09 y 5.61 para el directo, respectivamente. El resto de los funcionales predice en general un valor del gap subestimado. Por otro lado, los valores del band gap para $\mathrm{SiO}_{2}, \mathrm{GeO}_{2}$ y $\mathrm{SnO}_{2}$ predichos por el funcional GGA PBE son 5.7, 1.9 y $1.2 \mathrm{eV}$ respectivamente, mientras que sus valores experimentales son $8.9,4.7$ y $3.6 \mathrm{eV} \cdot[9]$

Es claro que, para los cinco sistemas estudiados, los cálculos con funcionales LDA y GGA subestiman en general el valor del band gap, mientras que en algunos casos los funcionales híbridos y funcionales con correcciones como la U de Hubbard o GW suelen arrojar valores más cercanos a los resultados experimentales.

\subsection{Metodología}

Con el fin de estudiar el efecto del intercambio exacto en la predicción del band gap de estos sistemas se realizaron cálculos de optimización de estructura utilizando el programa CRYSTAL14. En todos los sistemas fueron utilizados los funcionales de intercambio y correlación PBE y PBE0, así como variaciones de PBE0 con valores del parámetro $\alpha$, definido en la ecuación (3.15) del Capítulo 3, de $0.12,0.18,0.33$ y 0.50 . En todos los casos la optimización se realizó tanto para los parámetros de celda como para las coordenadas de la base. Como geometría 
inicial fueron utilizados datos cristalográficos experimentales.

Previo a los cálculos de optimización se realizaron cálculos para determinar la convergencia de la energía respecto al número de puntos $k$ utilizados dentro de la Zona de Brillouin, muestreados mediante el esquema de Monkhorst-Pack. En cuanto a las tolerancias utilizadas para la evaluación de las integrales se usaron en general los valores predeterminados, con la excepción del $\mathrm{MnO}$ donde se usó el valor recomendado en un artículo previo.[61] Para el $\mathrm{MnO}, \mathrm{SiO}_{2}, \mathrm{GeO}_{2}$ y $\mathrm{SnO}_{2}$ se usaron bases all electron, mientras que para el $\mathrm{ZrO}_{2}$ se usó un pseudopotencial con contribuciones relativistas, siendo obtenidas tanto las bases como el pseudopotencial de la base de datos de CRYSTAL.[62] En la Tabla 5.1 se muestran los números de puntos $k$ usados en la Zona Irreducible de Brillouin, así como las tolerancias usadas.

\begin{tabular}{lll}
\hline \hline Sistema & Puntos $k($ IBZ) & Tolerancias $\left(\mathrm{T}_{1}-\mathrm{T}_{5}\right)$ \\
\hline $\mathrm{MnO}$ & 260 & 687614 \\
$\mathrm{ZrO}_{2}$ & 170 & 666612 \\
$\mathrm{SiO}_{2}$ & 75 & 666612 \\
$\mathrm{GeO}_{2}$ & 75 & 666612 \\
$\mathrm{SnO}_{2}$ & 75 & 666612 \\
\hline \hline
\end{tabular}

Tabla 5.1: Datos de entrada para los cálculos de CRYSTAL14

En el caso específico del $\mathrm{MnO}$ se utilizó una supercelda con 8 celdas unitarias, con el fin de especificar el estado de espín de los átomos de Mn. La información experimental muestra que este sistema presenta un arreglo antiferromagnético tipo AF2;[61] verificamos que, para varios valores de $\alpha$, la estructura antiferromagnética AF2 es más estable que la AF1, como se muestra en la Figura 5.1.[63] En el resto de los casos se usó la celda unitaria correspondiente.

A partir de los sistemas optimizados con CRYSTAL14, usando la información de la función de onda resultante se obtuvieron las estructuras de bandas y las densidades de estados usando una herramienta de CRYSTAL; de las estructuras de bandas se calcularon los valores mínimos del band gap en cada caso. En las estruc- 


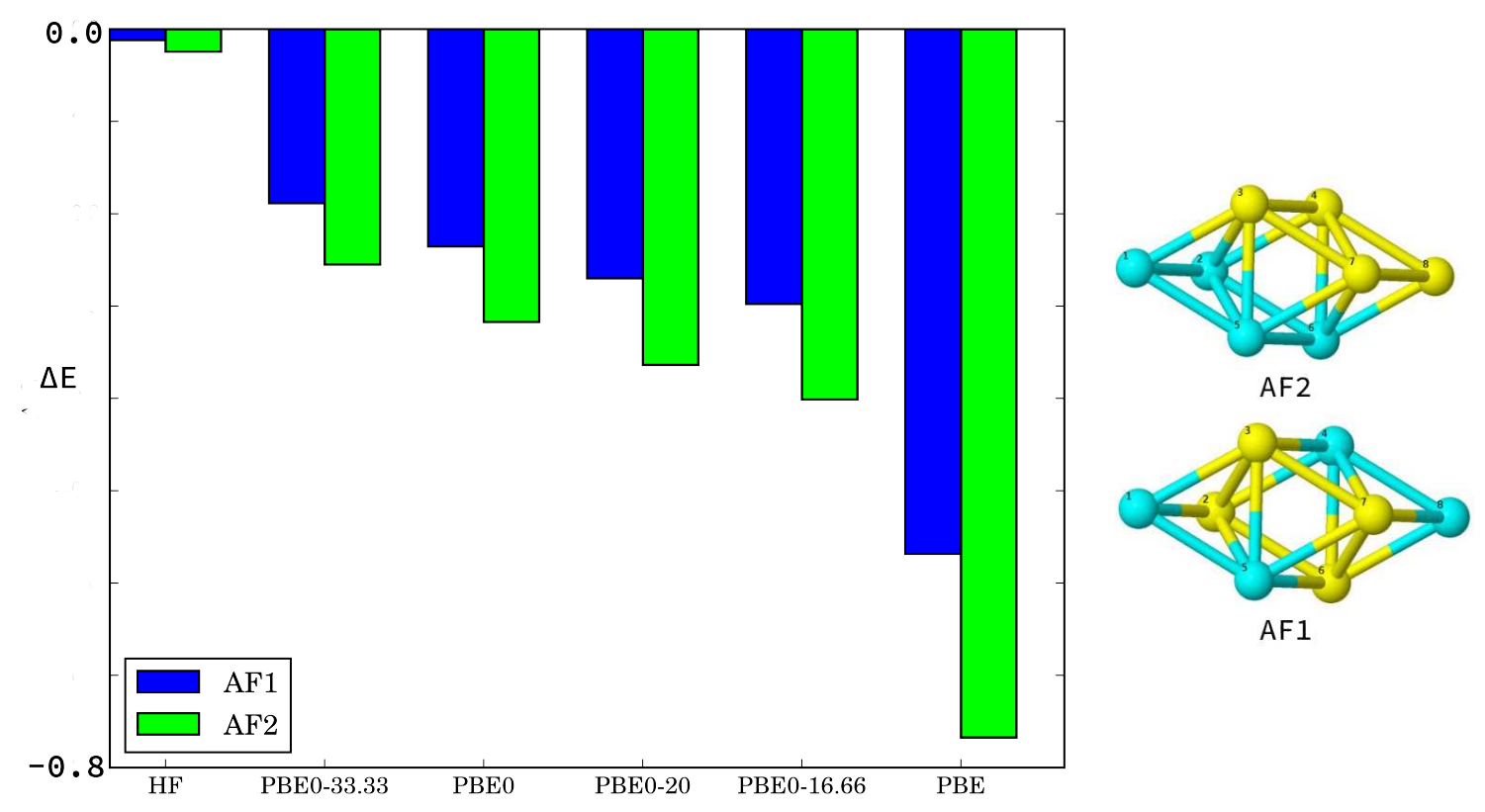

Figura 5.1: Izquierda: Energías relativas entre estados magnéticos del MnO. $\Delta E$ es la diferencia de energía entre el estado antiferromagnético y el estado ferromagnético. Derecha: Arreglos antiferromagnéticos AF1 y AF2 en el MnO. Las esferas de un mismo color representan átomos de Mn con el mismo espín. Por simplicidad no se muestran los oxígenos.

turas de bandas se seleccionaron rutas entre puntos de alta simetría en la Primera Zona de Brillouin, conforme al tipo de celda de cada sistema. En la densidad de estados se obtuvo tanto la densidad total como proyecciones sobre los átomos de la celda unitaria.

Partiendo de las geometrías optimizadas también fue calculado del Módulo de Bulto, a partir del cálculo de constantes elásticas. Dicho cálculo es realizado mediante segundas derivadas de la densidad energética con respecto a tensiones infinitesimales, y con un esquema automatizado para el cálculo del tensor elástico, todo esto implementado en CRYSTAL14.

Con los resultados obtenidos para el band gap se realizó para cada sistema una regresión lineal, a partir de la cual se determinaron los valores óptimos de $\alpha$ para reproducir el band gap experimental. 


\subsection{Parámetros geométricos y Módulo de Bulto}

Los valores obtenidos de los parámetros geométricos se muestran en las Tablas 5.2 y 5.3. En ambas tablas se aprecia que el aumento en el valor de $\alpha$ tiende a disminuir los parámetros de celda, de lo cual se concluye que el intercambio exacto tiende a reducir los parámetros de malla.

Por otro lado, los resultados del módulo de bulto se muestran en la Tabla 5.4. En la mayoría de los casos se observa que el valor de esta propiedad aumenta conforme aumenta el valor de $\alpha$, siendo la excepción el $\mathrm{ZrO}_{2}$.

En el caso del $\mathrm{ZrO}_{2}$ el valor obtenido del módulo de bulto parece ser relativamente constante con respecto a $\alpha$, con una variación máxima de $3 \mathrm{GPa}$. Los resultados obtenidos son cercanos a algunos valores previamente reportados, donde estiman valores experimentales de 95, 139 y $149 \mathrm{GPa}$,[64-66] y valores teóricos de 137 y 125 GPa.[67, 68] También existen en la literatura valores teóricos cercanos a otros valores experimentales notoriamente distintos a los valores aquí calculados, con resultados en el rango de 159-212 GPa.[69-72] En algunos de los reportes experimentales adjudican esta variaciones en el valor del módulo de bulto al uso de diferentes métodos de medición, al uso de pocos datos o de datos poco precisos, al nivel de pureza de la muestra usada, o a la técnica de preparación de la muestra.[72] Por argumentos similares pueden explicarse las variaciones encontradas para el módulo de bulto de los otros sistemas. 
Tabla 5.2: Parámetros de celda, en $\AA$, calculados para el $\mathrm{MnO}$ y el $\mathrm{ZrO}_{2}$ usando distintos valores de $\alpha$ en funcionales híbridos basados en el funcional PBE. En la última fila se muestran los parámetros de celda obtenidos con $\alpha_{o p t}$, y en paréntesis se encuentra el error relativo porcentual respecto al valor experimental. El valor de $\alpha_{o p t}$ es obtenido para reproducir el band gap experimental, como se muestra en la Tabla 5.6.

\begin{tabular}{c|c|ccccc}
\hline \hline$\alpha$ & $\mathrm{MnO}$ & \multicolumn{5}{|c}{$\mathrm{ZrO}_{2}$} \\
\hline & $a$ & $a$ & $b$ & $c$ & $\beta$ & $\mathrm{V}$ \\
\hline 0.00 & 6.345 & 5.2176 & 5.3087 & 5.3662 & 99.45 & 146.62 \\
0.12 & 6.325 & 5.1905 & 5.2927 & 5.3181 & 99.25 & 144.20 \\
0.18 & 6.316 & 5.1808 & 5.2822 & 5.3038 & 99.22 & 143.27 \\
0.25 & 6.306 & 5.1704 & 5.2702 & 5.2895 & 99.16 & 142.29 \\
0.33 & 6.294 & 5.1579 & 5.2587 & 5.2679 & 99.09 & 141.09 \\
0.50 & 6.271 & 5.1321 & 5.2382 & 5.2232 & 98.92 & 138.72 \\
\hline Exp. & $6.286[73]$ & 5.1505 & 5.2116 & 5.3173 & 99.23 & $140.88[74]$ \\
\hline$\alpha_{\text {opt }}$ & 6.309 & 5.1732 & 5.2750 & 5.2918 & 99.17 & 142.56 \\
& $(0.4)$ & $(0.4)$ & $(1.2)$ & $(0.5)$ & $(0.1)$ & $(1.2)$ \\
\hline \hline
\end{tabular}

Tabla 5.3: Parámetros de celda $\left(\mathrm{c}_{0}\right.$ y $\left.a\right)$, en $\AA$, para los sistemas con estructura tipo rutilo usando distintos valores de $\alpha$ en funcionales híbridos basados en el funcional PBE. En la última fila se muestran los parámetros de celda obtenidos con $\alpha_{o p t}$, y en paréntesis se encuentra el error relativo porcentual respecto al valor experimental. El valor de $\alpha_{o p t}$ es obtenido para reproducir el band gap experimental, como se muestra en la Tabla 5.6.

\begin{tabular}{c|cc|cc|cc}
\hline \hline$\alpha$ & \multicolumn{2}{|c|}{$\mathrm{SiO}_{2}$} & \multicolumn{2}{c|}{$\mathrm{GeO}_{2}$} & \multicolumn{2}{c}{$\mathrm{SnO}_{2}$} \\
\hline & $\mathrm{c}_{0}$ & $a$ & $\mathrm{c}_{0}$ & $a$ & $\mathrm{c}_{0}$ & $a$ \\
\hline 0.00 & 2.718 & 4.225 & 2.927 & 4.456 & 3.251 & 4.828 \\
0.12 & 2.703 & 4.198 & 2.906 & 4.418 & 3.229 & 4.795 \\
0.18 & 2.696 & 4.186 & 2.896 & 4.402 & 3.218 & 4.780 \\
0.25 & 2.688 & 4.174 & 2.886 & 4.384 & 3.208 & 4.765 \\
0.33 & 2.680 & 4.158 & 2.875 & 4.364 & 3.196 & 4.747 \\
0.50 & 2.663 & 4.129 & 2.853 & 4.330 & 3.176 & 4.710 \\
\hline Exp. & $2.662[75]$ & $4.181[75]$ & $2.865[75]$ & $4.402[75]$ & $3.187[75]$ & $4.738[75]$ \\
\hline$\alpha_{\text {opt }}$ & 2.687 & 4.172 & 2.887 & 4.387 & 3.218 & 4.780 \\
& $(0.9)$ & $(0.2)$ & $(0.8)$ & $(0.3)$ & $(1.0)$ & $(0.9)$ \\
\hline \hline
\end{tabular}


Tabla 5.4: Módulo de bulto en GPa, en función del parámetro $\alpha$ del funcional híbrido utilizado. En la última fila se muestran el módulo de bulto obtenido con $\alpha_{o p t}$, y en paréntesis el mayor y el menor error relativo porcentual de este resultado respecto al intervalo experimental. El valor de $\alpha_{o p t}$ es obtenido para reproducir el band gap experimental, como se muestra en la Tabla 5.6.

\begin{tabular}{c|c|c|c|c|c}
\hline \hline$\alpha$ & $\mathrm{MnO}$ & $\mathrm{ZrO}_{2}$ & $\mathrm{SiO}_{2}$ & $\mathrm{GeO}_{2}$ & $\mathrm{SnO}_{2}$ \\
\hline 0.00 & 160 & 129 & 213 & 279 & 182 \\
0.12 & 167 & 127 & 237 & 299 & 198 \\
0.18 & 170 & 129 & 248 & 308 & 206 \\
0.25 & 173 & 130 & 261 & 318 & 213 \\
0.33 & 176 & 129 & 275 & 330 & 224 \\
0.50 & 182 & 129 & 300 & 354 & 242 \\
\hline Exp. & $147-162$ & $95-212$ & $261,308,313$ & 267,251 & $203,205,212$ \\
& {$[76-79]$} & {$[64-66,70-72]$} & {$[80-82]$} & {$[83,84]$} & {$[85-87]$} \\
\hline$\alpha_{\text {opt }}$ & 172 & 130 & 263 & 316 & 206 \\
& $(17,6)$ & $(39,0)$ & $(16,0)$ & $(26,18)$ & $(3,0)$ \\
\hline \hline
\end{tabular}

\subsection{Estructura de bandas y densidad de estados}

Las estructuras de bandas obtenidas, en compañía de las densidades de estados (DOS) correspondientes se muestran en las Figuras ??????????

En general se observa que un aumento en la contribución del intercambio exacto en el funcional de intercambio y correlación aumenta la brecha entre la banda de conducción y la banda de valencia. Otra característica constante entre estos sistemas, con excepción del $\mathrm{MnO}$, es que la forma de la estructura de bandas y de la densidad de estados total se mantiene prácticamente idéntica para cualquier valor de $\alpha$. Las estructuras de bandas de los sistemas con elementos del grupo IVA presenta varias similitudes tanto en la parte de valencia como en la de conducción.

En el caso de las densidades de estados del $\mathrm{ZrO}_{2}$ (Figura 5.4), las proyecciones de la DOS sobre los orbitales $4 d$ de $\mathrm{Zr}$ y los $2 s 2 p$ del O muestran que los primeros contribuyen más a la banda de conducción, mientras que los primeros lo hacen en la banda de valencia. 
Para el $\mathrm{SnO}_{2}$ se observa un comportamiento similar para los orbitales $2 s 2 p$ del $\mathrm{O}$, pero los orbitales $4 d$ del Sn no contribuyen ni en la banda de valencia ni en la de conducción. Por otro lado, para $\mathrm{SiO}_{2}$ y $\mathrm{GeO}_{2}$ no se observa contribución por parte de los orbitales $3 s 3 p$ del $\mathrm{Si}$, los $3 d$ del Ge, ni los $2 s 2 p$ de los $\mathrm{O}$ de ambos sistemas. Proyecciones sobre los orbitales desocupados incluidos en las bases de estos tres sistemas muestran que son estos orbitales los que conforman tanto la banda de conducción como la banda de valencia.

En el caso el $\mathrm{MnO}$ se observan cambios notables en la forma de la estructura de bandas y la DOS. Las bandas de conducción y las de valencia se distribuyen en un intervalo mayor de energías conforme se aumenta el valor de $\alpha$, resultando en bandas menos localizadas. Entre PBE y el resto de los funcionales se observa un cambio en la naturaleza del band gap menor, pasando de ser un gap directo a ser indirecto. En estos casos con $\alpha \neq 0$ se observa un pico en la banda de valencia con un mínimo en el punto $\Gamma$, cuya diferencia con la región más poblada de bandas aumenta con $\alpha$, mientras que el máximo de la región de valencia está en el punto $L$. En la DOS total esto se refleja en una baja densidad de estados en el intervalo de energía asociado a dicho pico.

En cuando a las DOS proyectadas, para todos los casos tanto en la banda de conducción como en la de valencia predominan los orbitales $3 d$ del Mn frente a los $2 s 2 p$ del $\mathrm{O}$. Un fenómeno interesante es el efecto del valor de $\alpha$ en las proyecciones en la banda de valencia, donde se aprecia que la contribución de los estados $3 d$ del $\mathrm{MnO}$ disminuye con el valor de $\alpha$, acercándose bastante la proyección de los estados $2 s 2 p$ del O. Es claro que para este sistema es notable el cambio en la descripción de la naturaleza de la región de valencia y conducción respecto al valor de $\alpha$. 

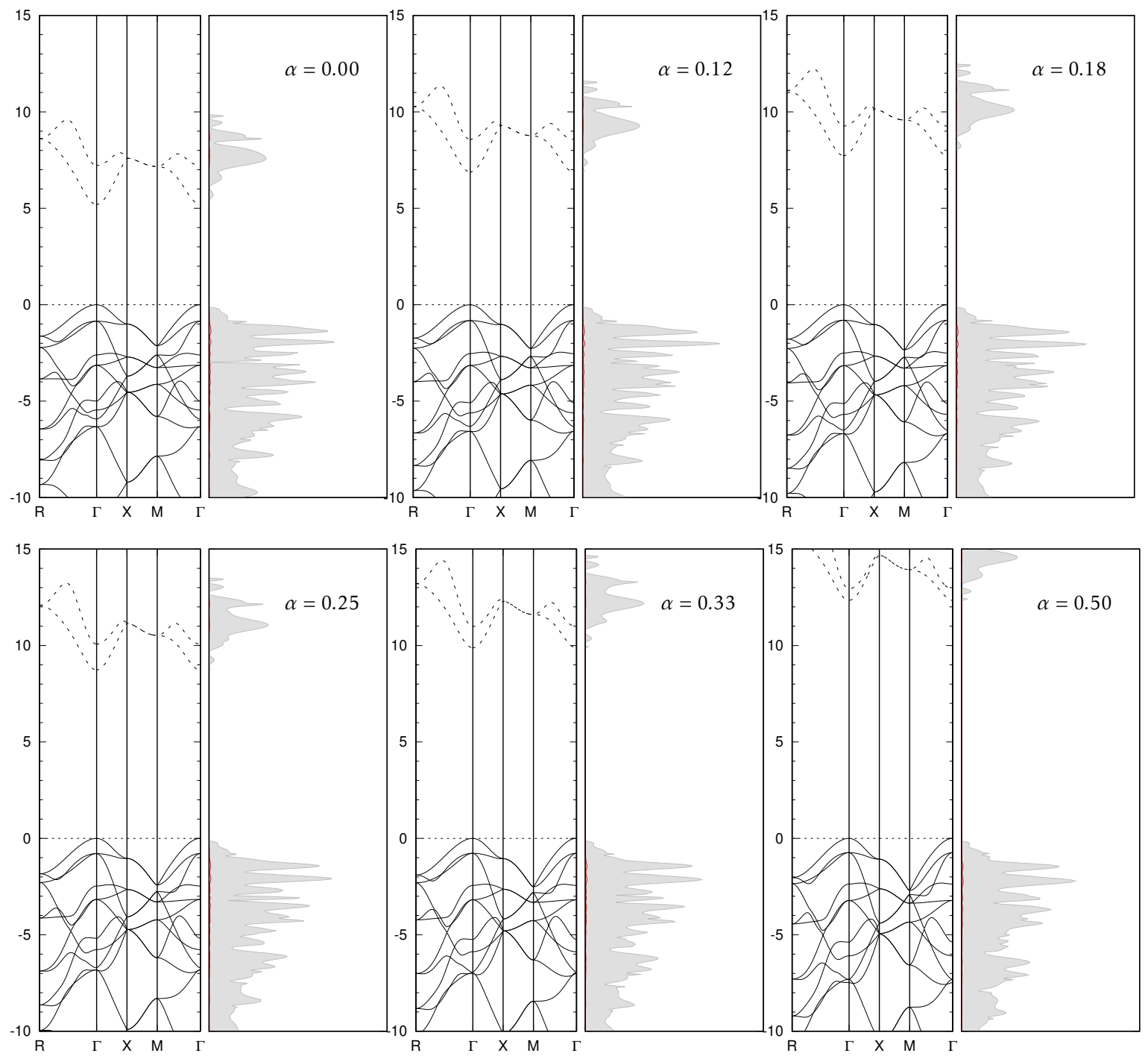

Figura 5.2: Estructuras de bandas y densidades de estados para el $\mathrm{SiO}_{2}$ con diferentes valores de $\alpha$. En el eje ordenado se muestra la energía relativa al máximo de la banda de valencia (linea punteada horizontal) en eV. La curva azul es la DOS proyectada sobre los orbitales $3 s 3 p$ del metal y la curva roja la DOS proyectada sobre los orbitales $2 s 2 p$ del oxígeno. 

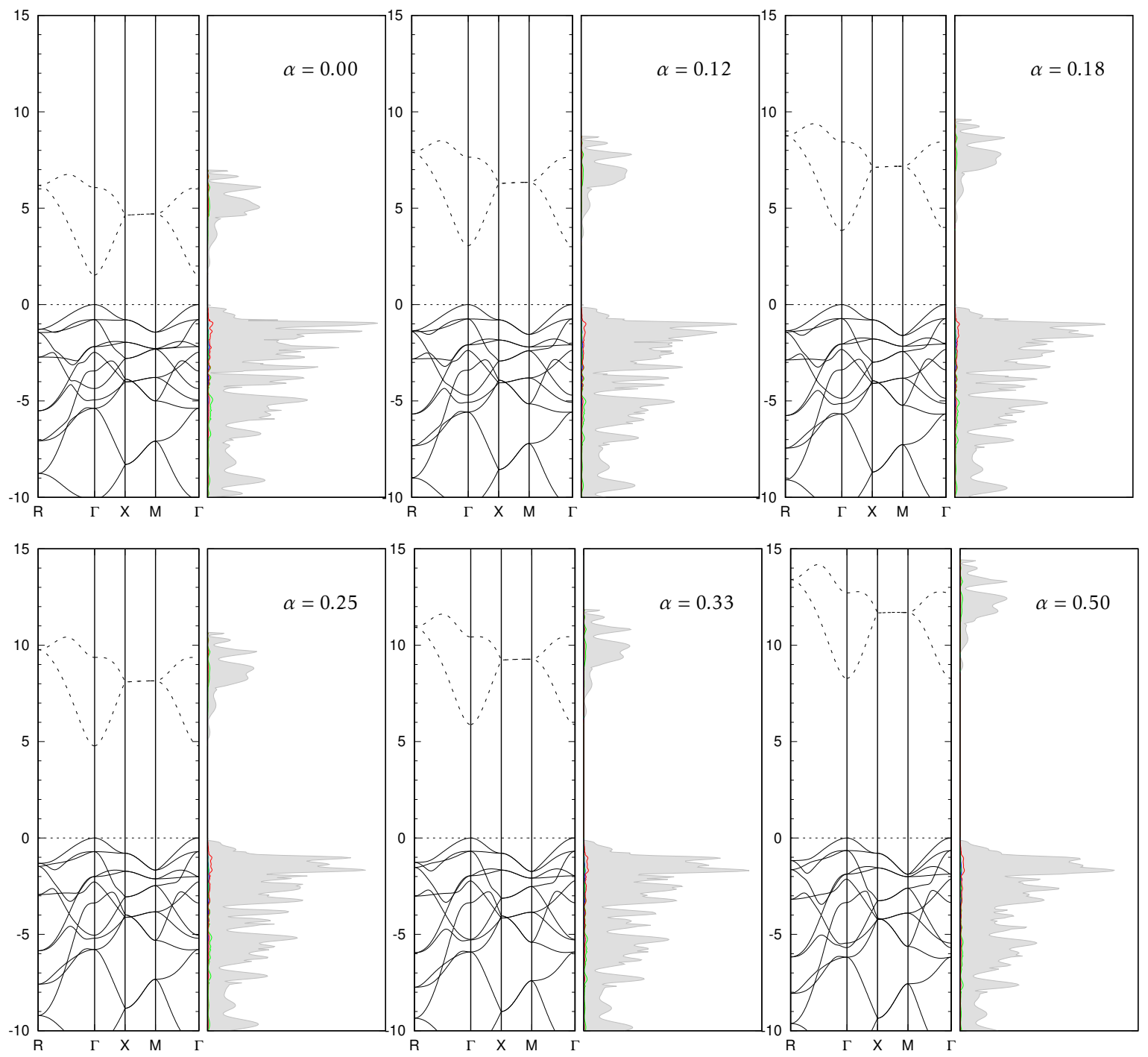

Figura 5.3: Estructuras de bandas y densidades de estados para el $\mathrm{GeO}_{2}$ con diferentes valores de $\alpha$. En el eje ordenado se muestra la energía relativa al máximo de la banda de valencia (linea punteada horizontal) en eV. La curva azul es la DOS proyectada sobre los orbitales $3 d$ del metal y la curva roja la DOS proyectada sobre los orbitales $2 s 2 p$ del oxígeno. 

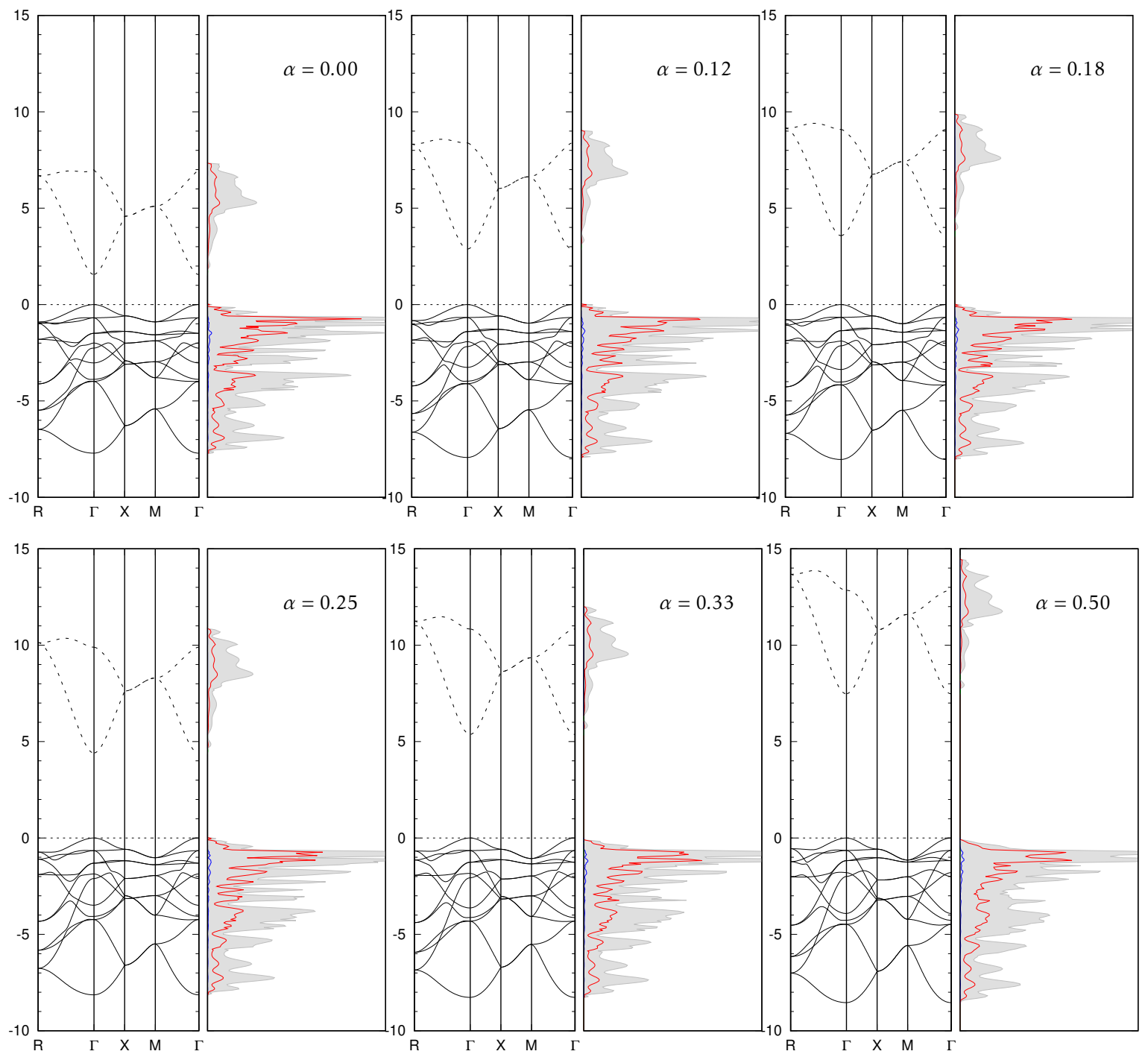

Figura 5.4: Estructuras de bandas y densidades de estados para el $\mathrm{SnO}_{2}$ con diferentes valores de $\alpha$. En el eje ordenado se muestra la energía relativa al máximo de la banda de valencia (linea punteada horizontal) en eV. La curva azul es la DOS proyectada sobre los orbitales $4 d$ del metal y la curva roja la DOS proyectada sobre los orbitales $2 s 2 p$ del oxígeno. 

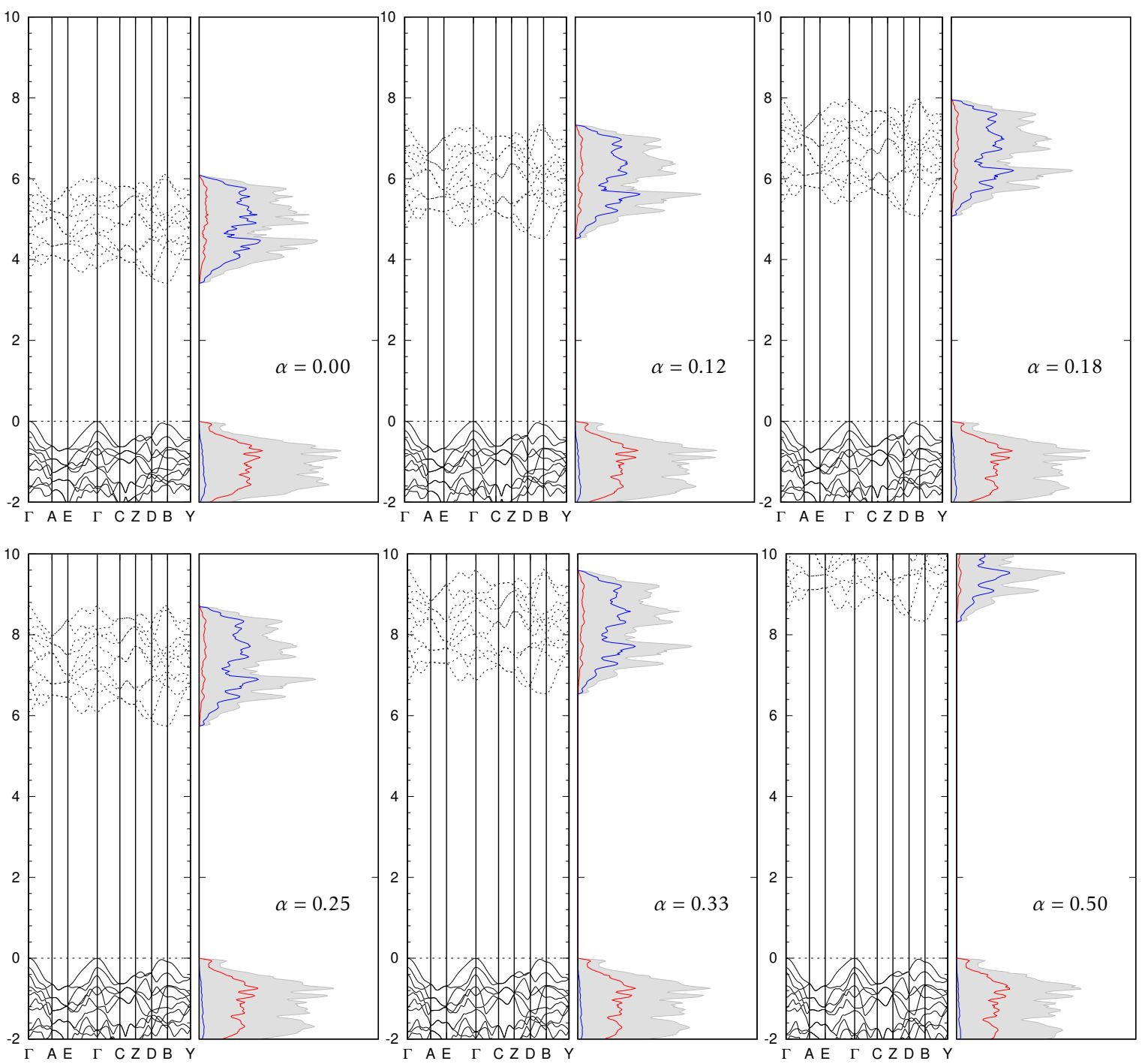

Figura 5.5: Estructuras de bandas y densidades de estados para el $\mathrm{ZrO}_{2}$ con diferentes valores de $\alpha$. En el eje ordenado se muestra la energía relativa al máximo de la banda de valencia (linea punteada horizontal) en eV. La curva azul es la DOS proyectada sobre los orbitales $4 d$ del metal y la curva roja la DOS proyectada sobre los orbitales $2 s 2 p$ del oxígeno. 

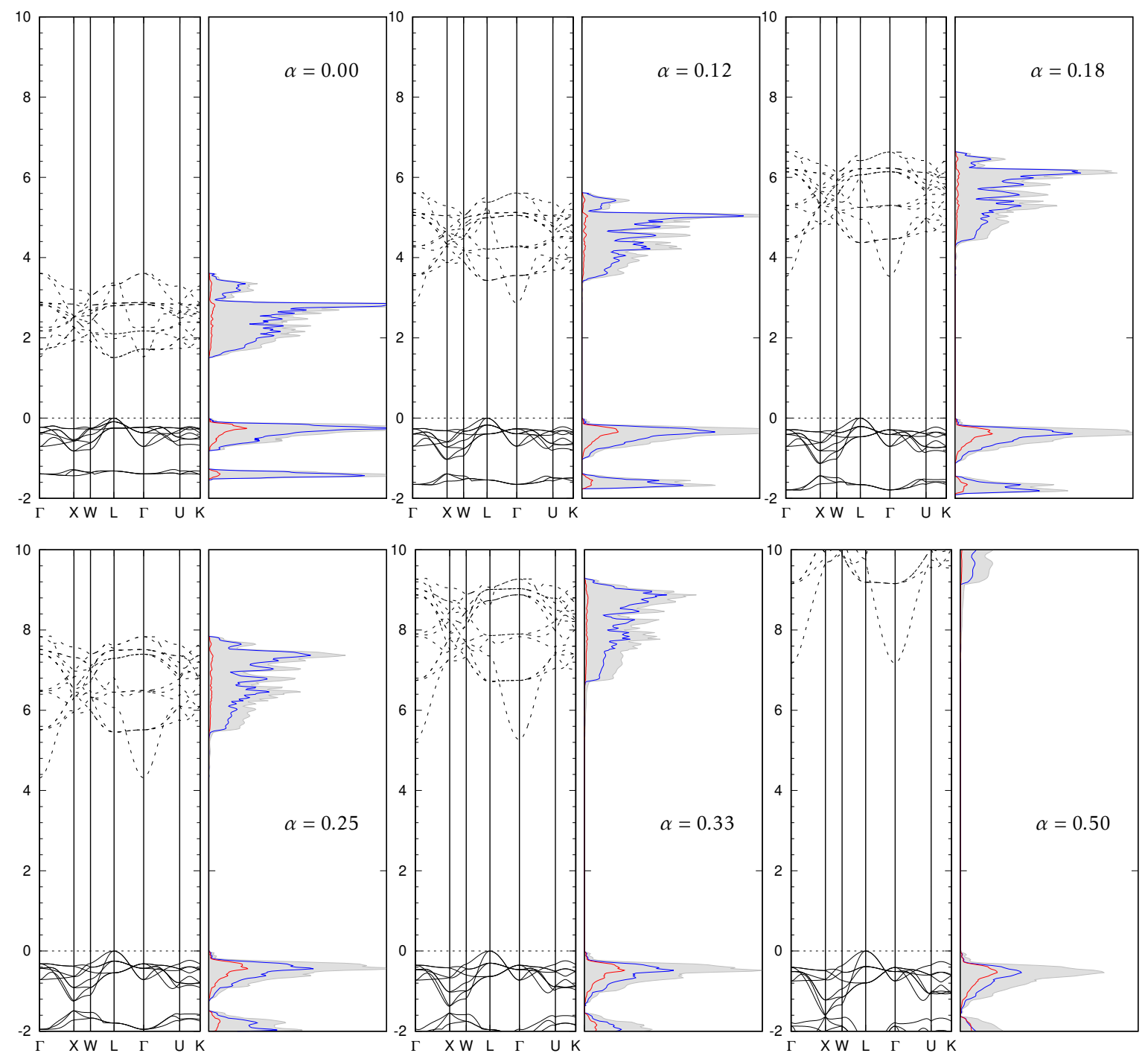

Figura 5.6: Estructuras de bandas y densidades de estados (DOS) para el $\mathrm{MnO}$ con diferentes valores de $\alpha$. En el eje ordenado se muestra la energía relativa al máximo de la banda de valencia (linea punteada horizontal) en eV. La curva azul es la DOS proyectada sobre los orbitales $3 d$ del metal y la curva roja la DOS proyectada sobre los orbitales $2 s 2 p$ del oxígeno. Tanto las estructuras de bandas como las DOS del $\mathrm{MnO}$ son equivalentes para los electrones con espín $\alpha$ como para los electrones con espín $\beta$

\subsection{Band gap}

Los valores obtenidos para el band gap se muestran en la Tabla 5.5. En general un aumento en la contribución del intercambio exacto en el funcional de 
intercambio y correlación aumenta el valor del band gap. Tal como es reportado previamente en la literatura, PBE tiende a subestimar el gap de estos sistemas, resultando en el menor band gap calculado para cada sistema. También en acuerdo con reportes previos, PBE0 da resultados aceptables para el valor del gap en todos los casos.

En casi todos los sistemas estudiados el band gap resultó ser un gap directo, encontrándose el mínimo de la banda de conducción y el máximo de la banda de valencia en el mismo punto $k$. En el caso del $\mathrm{ZrO}_{2}$ se puede apreciar en la Figura 5.5 que el máximo de la banda de valencia está ligeramente desplazado del punto $k$ especial $B$ donde se encuentra el mínimo de la banda de conducción, pero ya que tanto el desplazamiento como el cambio en energía respecto al punto $B$ es pequeño sólo se reporta un valor del gap. En el caso del $\mathrm{MnO}$ la diferencia entre gap directo e indirecto es apreciable, por lo que se reportan ambos valores.

Tabla 5.5: Band gap, $E_{g}(\mathrm{eV})$, en función de $\alpha$.

\begin{tabular}{c|c|c|c|cr|c}
\hline \hline$\alpha$ & $\mathrm{SiO}_{2}$ & $\mathrm{ZrO}_{2}$ & $\mathrm{GeO}_{2}$ & \multicolumn{2}{|c|}{$\mathrm{MnO}$} & $\mathrm{SnO}_{2}$ \\
& & & & Directo Indirecto & \\
\hline 0.00 & 5.19 & 3.41 & 1.52 & \multicolumn{2}{|c}{1.51} & 1.56 \\
0.12 & 6.88 & 4.52 & 3.05 & 3.13 & 2.86 & 2.88 \\
0.18 & 7.73 & 5.08 & 3.84 & 3.83 & 3.53 & 3.57 \\
0.25 & 8.73 & 5.74 & 4.78 & 4.64 & 4.32 & 4.40 \\
0.33 & 9.88 & 6.54 & 5.88 & 5.62 & 5.26 & 5.37 \\
0.50 & 12.34 & 8.34 & 8.28 & 7.54 & 7.17 & 7.48 \\
\hline Exp. & $8.9[88]$ & $5.3-5.83[89,90]$ & $4.68[91]$ & \multicolumn{4}{|c}{$4.1[92]$} & $3.6[93]$ \\
\hline \hline
\end{tabular}

Con los valores obtenidos para el gap se realizaron ajustes lineales de dichos valores con respecto al parámetro $\alpha$ para cada sistema, como se muestra en la Figura 5.7. En la Tabla 5.6 se muestran los parámetros obtenidos para cada ajuste; en todos los casos el ajuste resulta ser adecuado, tal como muestran los valores de $\mathrm{R}^{2}$.

Mediante los ajustes realizados fueron obtenidos valores de $\alpha$ tales que fuera reproducido el valor del band gap experimental, o el valor promedio de los gaps 
experimentales encontrados dado el caso. Usando este valor $\alpha_{\text {opt }}$ se realizaron cálculos equivalentes a los efectuados con otros valores de $\alpha$, reportándose los resultados correspondientes al final de las Tablas 5.2, 5.3 y 5.4. En el caso del MnO solamente se realizaron dichos cálculos para el valor de $\alpha_{\text {opt }}$ obtenido a partir de los gaps indirectos.

Los valores del band gap usando $\alpha_{\text {opt }}$ se reportan en la Tabla 5.6, resultando en valores de gap idénticos a los experimentales tal como se esperaba. Respecto a los parámetros geométricos el uso de este valor resultó en valores en acuerdo razonable con los resultados experimentales, tal como muestra el error relativo porcentual mostrado en las Tablas 5.2 y 5.3. En el caso del módulo de bulto (Tabla 5.4) el valor de este error en algunos casos es considerable, pero aún así los valores resultan ser cercanos a los reportados en la literatura.

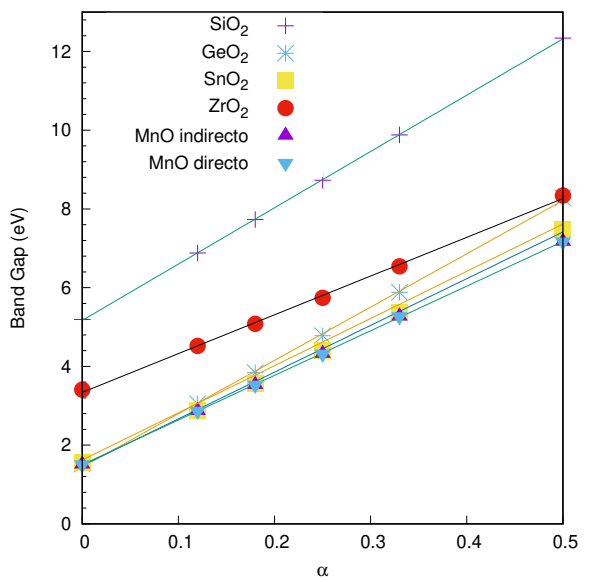

Figura 5.7: Ajustes lineales del band gap $(\mathrm{eV})$ respecto a $\alpha$ para cada sistema.

\begin{tabular}{llllll}
\hline & $\mathrm{b}$ & $\mathrm{m}$ & $\mathrm{R}$ & $\alpha_{\text {opt }}$ & $\mathrm{Gap}\left(\alpha_{\text {opt }}\right)$ \\
\hline $\mathrm{SiO}_{2}$ & 5.17 & 14.31 & 1.000 & 0.2607 & 8.90 \\
$\mathrm{ZrO}_{2}$ & 3.34 & 9.85 & 0.999 & 0.2259 & 5.52 \\
$\mathrm{GeO}_{2}$ & 1.44 & 13.54 & 0.999 & 0.2393 & 4.68 \\
$\mathrm{MnO}-\mathrm{i}$ & 1.49 & 11.30 & 0.999 & 0.2317 & 4.09 \\
$\mathrm{MnO}-\mathrm{d}$ & 1.62 & 11.98 & 0.999 & 0.2068 & - \\
$\mathrm{SnO}_{2}$ & 1.48 & 11.87 & 0.999 & 0.1786 & 3.60 \\
\hline
\end{tabular}

Tabla 5.6: Parámetros del ajuste lineal entre $\alpha$ y el band gap calculado. La columna $\alpha_{o p t}$ muestra el valor de $\alpha$ ajustando con las relaciones lineales obtenidas para reproducir el band gap experimental o, para el $\mathrm{ZrO}_{2}$, el promedio de éste. La columna $\operatorname{Gap}\left(\alpha_{\text {opt }}\right)$ muestra el valor calculado del band gap, en $\mathrm{eV}$, usando $\alpha_{\text {opt }}$. En el caso del $\mathrm{MnO}$, -i indica los valores del ajuste usando los gaps indirectos, $\mathrm{y}-\mathrm{d}$ los valores usando los gaps directos. 


\subsection{Conclusiones}

Los resultados obtenidos confirman que, tal como ya se ha reportado, por medio de la relación lineal existente entre el band gap y el parámetro $\alpha$ asociado al intercambio exacto de funcionales híbridos de intercambio y correlación de un parámetro es posible determinar el valor óptimo de $\alpha$ que reproduce el gap experimental, manteniendo buenos resultados para la geometría y las propiedades mecánicas del sistema. Un punto a notar es que para los sistemas estudiados en esta tesis el uso del funcional PBE (equivalente a $\alpha=0$ ) no es recomendable, lo que concuerda con lo que hemos publicado recientemente para átomos confinados por paredes penetrables, donde el modelo de átomos confinados simula razonablemente bien lo que acontece en un cristal en ciertos sistemas.[94]

Con lo mostrado en este trabajo, es claro que los efectos debidos al intercambio electrónico resultan de gran importancia para la correcta descripción de propiedades electrónicas de estos óxidos metálicos. Además, estos resultados, publicados recientemente $[95]^{1}$, muestran al igual que otros que no existe un valor universal de $\alpha$ para describir cualquier sistema, siendo necesario ajustarlo para cada caso.

\subsection{Perspectivas}

Dado que podemos determinar el valor óptimo de $\alpha$ para reproducir propiedades de estos óxidos, es posible explorar cuales son los efectos de distintos tipos de defectos sobre estos sistemas partiendo de este resultado. Algunos de los sistemas que han sido probados con esta idea son el MnO con una vacancia de oxígeno, y el $\mathrm{ZrO}_{2}$ con Ti como sustituyente. En ambos casos es importante conseguir información experimental que respalde los resultados obtenidos. Con esta información de respaldo sería posible establecer si el valor de $\alpha$ obtenido para el sistema puro es

\footnotetext{
${ }^{1}$ Con excepción de los resultados para $\mathrm{ZrO}_{2}$
} 
adecuado para describir sistemas con bajas concentraciones de defectos y, dado el caso, hasta qué concentración este valor de $\alpha$ sigue siendo adecuado para estudiar estos sistemas. 
Si esta operación se realiza correctamente en paralelo, estarán ejecutándose al mismo tiempo y de forma independiente las siguientes tareas:

$$
\mathrm{j}[1]=1, \quad \mathrm{j}[2]=2, \quad \ldots, \quad \mathrm{j}[\mathrm{N}-1]=\mathrm{N}-1, \quad \mathrm{j}[\mathrm{N}]=\mathrm{N}
$$

Si se utiliza un GPU, estos procesos individuales son llamados hilos. Dado el ejemplo previo, en la GPU se ejecutarán $\mathrm{N}$ hilos en paralelo, siempre que $\mathrm{N}$ sea menor o igual al número máximo de hilos que admite el dispositivo.

OpenACC está basado en el uso de directivas, las cuales son líneas de código que modifican el comportamiento del compilador. En OpenACC para Fortran, estas directivas tienen la estructura ! \$acc directiva opciones. Estas directivas se dividen en tres tipos: de ejecución, de control de datos y de sincronización.

Las directivas más importantes son las de ejecución, ya que éstas señalan las regiones de código que se desean ejecutar en el dispositivo. Existen dos principales: la directiva parallel, que indica al compilador que la región es paralelizable, y la directiva kernels, que indica al compilador que paralelice la región sólo si puede determinar que es posible. Al resultar una opción más cauta, en un inicio resulta recomendable utilizar la directiva kernels para que el programa resultante tenga un funcionamiento correcto. Para delimitar el alcance de la directiva se utiliza !\$acc end directiva; en algunos casos este alcance es determinado por el compilador y no es necesario añadir esta línea al programa.

Las directivas de ejecución se colocan antes de un ciclo Do. Por ejemplo:

$$
\begin{aligned}
& \text { ! \$acc kernels } \\
& \text { Do } \mathrm{i}=1, \mathrm{~N} \\
& \mathrm{j}[\mathrm{i}]=\mathrm{i}
\end{aligned}
$$

\section{Enddo}

$$
\text { ! \$acc end kernels }
$$

, lo cual indica al compilador que intente paralelizar el código entre las directivas. 
Si el compilador determina que la región es paralelizable producirá las instrucciones correspondientes para su ejecución de esta forma, y en caso contrario producirá instrucciones que serán ejecutadas en el dispositivo pero de forma serial. En adelante se utilizará región para indicar la región del código afectada por las directivas de ejecución.

En cuanto al tratamiento de datos, el compilador tiene cierta capacidad de interpretar qué movimientos, tales como copia de arreglos hacia y desde el dispositivo, son requeridos para la ejecución en el dispositivo. Estos movimientos dependen, además del código de la región, del dispositivo que se intente utilizar: si es un procesador multicore éste puede acceder a la memoria del equipo que contiene al dispositivo, también llamado huésped. Sin embargo, si se utiliza un GPU dicho dispositivo no tiene acceso directo a la memoria del huésped, por lo que deben realizarse movimientos de datos entre huésped y dispositivo.

También es posible indicarle al compilador algunas instrucciones o detalles sobre las variables contenidas en la región, tales como:

- Las variables son independientes entre cada ciclo.

- Debe reservarse memoria para almacenar un arreglo.

- Las variables deben ser copiadas desde el huésped y no necesitan copiarse al huésped al final de la región.

- Las variables no necesitan ser copiadas del huésped y al final de la región deben copiarse al huésped.

- Las variables son copiadas del huésped al inicio y al huésped al final.

Estas propiedades son generalmente indicadas en conjunto con la directiva de cómputo. Por ejemplo, si se desea que el arreglo $j$ solamente sea copiado hacia el huésped al final de la región, se utiliza la opción copyin: 


$$
\begin{aligned}
& \text { ! \$acc kernels copyin }(j) \\
& \text { Do } i=1, N \\
& j[i]=i
\end{aligned}
$$

\section{Enddo}

\section{! \$acc end kernels}

Algunas opciones más avanzadas de OpenACC fueron usadas en el caso de las modificaciones realizadas a Crystal. Si la región a paralelizar realiza llamados a otras rutinas es necesario indicarle al compilador que genere versiones de éstas para su ejecución en el dispositivo. Con este fin se utiliza la directiva de ejecución routine.

También es necesario un tratamiento especial para las variables globales involucradas en la región a paralelizar. En el caso especial de Fortran, las variables de tipo Common Block no tienen soporte de OpenACC dentro de funciones con la directiva routine. Para el caso de las variables contenidas dentro de Módulos sí existe una opción, utilizando la directiva de control de datos declare. Las variables señaladas en esta directiva requieren de un mayor seguimiento que el resto, ya que estas requieren que se señale explícitamente cual es la última asignación que tuvieron antes y dentro de la región (con la directiva update), y en el caso de arreglos de tamaño dinámico es necesario que el compilador detecte explícitamente la instrucción de reservado de memoria.

OpenACC posee tres niveles de paralelismo: gangs, workers y vectors. La relevancia de estos niveles radica en que se puede considerar que uno está contenido en el anterior, encontrándose entonces un gang compuesto de workers que a su vez contienen vectors. En el caso de que las ciclos Do estén anidados, se puede utilizar esta jerarquía de niveles para agrupar, en el caso de un GPU, los hilos que ejecutarán en paralelo los ciclos más externo, y dentro de estos hilos agrupar aquellos que realizarán los ciclos internos. Por ejemplo: 
! \$acc kernels copyin(a)

Do $\mathrm{i}=1, \mathrm{~N}$

Do $\mathrm{j}=1, \mathrm{M}$

Do $\mathrm{k}=1, \mathrm{~L}$

$a[i, j, i]=i^{\wedge} 2+j^{\wedge} 2+k^{\wedge} 2$

\section{Enddo}

Enddo

\section{Enddo}

\section{! \$acc end kernels}

El ciclo que va sobre el índice $i$ puede ejecutarse al nivel de gangs, el ciclo sobre $j$ al nivel de workers y el ciclo sobre $k$ al nivel de vectores. Si bien es posible indicar qué nivel de paralelismo se utilizará para cada Do, el compilador automáticamente realiza un análisis y propone los niveles de paralelismo que considera viables para las distintas operaciones cíclicas contenidas en la región indicada por una directiva de ejecución. 

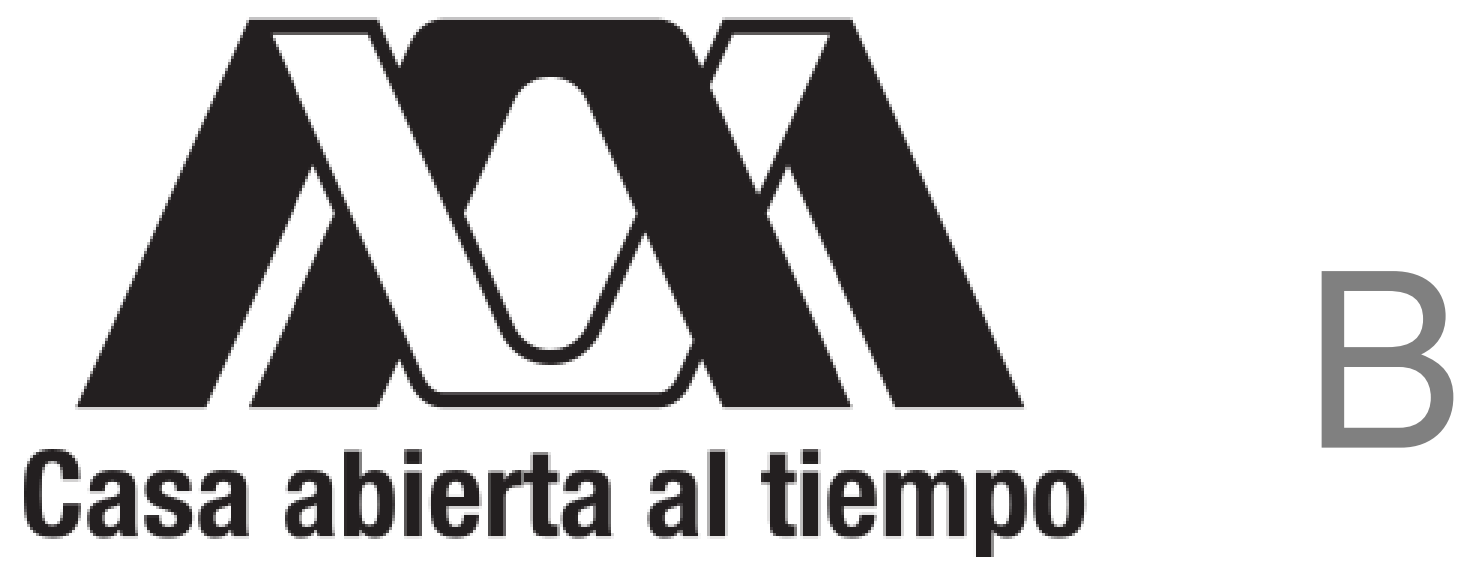

Artículos publicados 


\title{
Importance of one-parameter hybrid exchange-correlation functionals in band gaps of transition metal and metalloid oxides
}

\author{
Alejandra M. Navarrete-López ${ }^{1} \cdot$ Marcos Rivera-Almazo $^{2} \cdot$ Jorge Garza $^{2} \cdot$ Rubicelia Vargas $^{2}$ (D) \\ Received: 29 October 2017 / Accepted: 12 February 2018 / Published online: 24 February 2018 \\ (c) Springer-Verlag GmbH Germany, part of Springer Nature 2018
}

\begin{abstract}
In this work, the effect of the exact exchange in hybrid functionals based on one parameter is explored over the electronic structure of $\mathrm{Ti}_{2} \mathrm{O}_{3}, \mathrm{~V}_{2} \mathrm{O}_{3}, \mathrm{Cr}_{2} \mathrm{O}_{3}, \mathrm{Fe}_{2} \mathrm{O}_{3}, \mathrm{MnO}, \mathrm{SiO}_{2}, \mathrm{GeO}_{2}$, and $\mathrm{SnO}_{2}$, such that oxides with different nature are included in this data set. Structural parameters and magnetic states of these oxides are reproduced according to experimental information, which are discussed in the context of the exact exchange inclusion. Several exchange-correlation functionals are considered to reach this goal, two of them, HSE06 and B1WC, which were designed ad hoc to study metal oxides are contrasted with hybrid exchange-correlation functionals that contain a fraction $(\alpha)$ of the exact exchange, like PBE0. Thus, in this work, hybrid functionals where $\alpha$ is varied systematically provide a linear relationship between band gap and $\alpha$, which gives one way to match the theoretical band gap with experimental information. If this optimum $\alpha$ is used to predict cell parameters or bulk modulus, then the corresponding results are close to experimental data. For the systems considered in this work, all-electron calculations were performed using a periodic ab initio code, which uses Gaussian localized basis set functions for the expansion of Bloch orbitals by linear combinations of atomic orbitals.
\end{abstract}

Keywords Semiconductors $\cdot$ Metal oxides $\cdot$ Exact exchange $\cdot$ Band gap $\cdot$ Hybrid exchange-correlation functionals

\section{Introduction}

Transition metal oxides are probably the most interesting kind of solids, and the variety of electronic and magnetic properties are noteworthy. The unusual properties of these materials are due to the nature of outer d electrons of transition metals. There are some oxides of $3 d$ transition metals involved to produce materials with multiple technological applications, like batteries, catalyzers, or solar cells, that present appreciable band gap $\left(E_{\mathrm{g}}\right)$. In particular, for solar

Published as part of the special collection of articles "In Memoriam of Claudio Zicovich".

Rubicelia Vargas

ruvf@xanum.uam.mx

1 División de Ciencias Básicas e Ingeniería, Departamento de Ciencias Básicas, Universidad Autónoma MetropolitanaAzcapotzalco, Av. San Pablo Xalpa 180, Reynosa

Tamaulipas, Azcapotzalco, C. P. 02200 Mexico City, Mexico

2 División de Ciencias Básicas e Ingeniería, Departamento de Química, Universidad Autónoma Metropolitana-Iztapalapa, San Rafael Atlixco 186, Col. Vicentina, Iztapalapa, C. P. 09340 Mexico City, Mexico cells, the design of materials with a band gap to maximize the absorption of incident sunlight is an important issue. Evidently, the theoretical estimation of the band structure for these materials is one fundamental challenge. The Density Functional Theory (DFT), in its Kohn-Sham (KS) version, is the most used theoretical method to study these systems. However, the DFT-KS method within local density approximation (LDA) or generalized gradient approximation (GGA) presents substantial difficulties to describe the band gap correctly for many systems. For LDA, the difference between the bottom of the conduction band and the top of the valence band is smaller than those band gaps reported experimentally. For example, for hematite $\left(\alpha-\mathrm{Fe}_{2} \mathrm{O}_{3}\right)$, this method predicts narrow gaps, among $0.32-0.75 \mathrm{eV}[1,2]$, which are distant from the experimental information $(2.0-2.7 \mathrm{eV})[3,4]$. This wrong behavior is particularly severe for $\mathrm{Ti}_{2} \mathrm{O}_{3}$; LDA predicts a metallic behavior [5] while experiments show that this is an insulator at low temperature $[6,7]$. By the side of GGA for this kind of materials, the band gap predictions are not notably improved with regard to LDA. Rollmann et al. [2] showed that for hematite, GGA underestimates this quantity. For chromia, $\mathrm{Cr}_{2} \mathrm{O}_{3}$, this approximation predicts one small band gap (1.2 eV) with respect to experimental 
information (3.4 eV) [8]. Similar results are observed when GGA is used in $\mathrm{MnO}$ [9] and other metal oxides [10, 11].

By another side, other kind of oxides that has been studied for their applications in many technological branches is metalloids such as silicon $\left(\mathrm{SiO}_{2}\right)$ and germanium dioxides $\left(\mathrm{GeO}_{2}\right)$ that are relevant materials. Experimental techniques show a large $E_{\mathrm{g}}$ in their electronic structure, which is a quality of these two oxides [12-15]. In these cases, no outer d electrons are present; however, for $\mathrm{SiO}_{2}$ and $\mathrm{GeO}_{2}$, the results obtained by LDA or GGA are far from the experimental information [16-19].

The band gap and magnetic moments from LDA or GGA are corrected using both $\mathrm{LDA}+\mathrm{U}$ and GGA+U approximations [20-23]. The main problem with these approximations is that they require two empirical parameters: the Hubbard, $U$ and the Hund, $J$ [24, 25]. These corrections provide a reasonable agreement with experimental values when these empirical parameters are fitted adequately [26]. Unfortunately, even with the efforts devoted on the $U$ method, the band gap differs in an important way from true quasiparticle gap (measured, e.g., in photoemission and inverse photoemission experiments) [27].

The wrong asymptotic behavior and the non-discontinuity of LDA and GGA exchange-correlation potentials are the main problems involved in the band gap prediction [28-31]. There are several reports where orbital-dependent exchangecorrelation potentials alleviate some problems [32-34]; in particular, when a fraction of the exact exchange is incorporated in exchange-correlation approximations, the performance of the resulting functionals improve the band gap description $[35,36]$.

The basic idea of some hybrid exchange-correlation functionals is to include a fraction, $\alpha$, of the exact exchange (EEX)

$E_{\mathrm{xc}}^{\mathrm{hybrid}}=E_{\mathrm{xc}}^{\mathrm{approx}}+\alpha\left(\mathrm{EEX}-E_{x}^{\mathrm{approx}}\right)$,

where $E_{x}^{\text {approx }}$ and $E_{\mathrm{xc}}^{\text {approx }}$ represent approximations to exchange $(X)$ and exchange-correlation (XC) functionals, respectively. It is worth to note that sometimes $\alpha$ is related to $1 / n$ with $n$ as an integer. Some reports indicate that $n=2,3,4$ and 5 are well justified [37-39], although other values for $\alpha$ have been also discussed for periodic systems [40]. The PBE0 XC functional with $n=4$ is the most popular approximation in this $\mathrm{XC}$ family that uses only one parameter [41, 42]. For periodic systems, PBE0 exhibits a good performance predicting geometries. However, several issues in periodic systems have been observed for this exchange-correlation functional, for example, vibrational frequencies are overestimated [43, 44], and band gaps are described incorrectly for systems where atoms with d orbitals are present. Thus, the good performance exhibited by PBE0 over finite systems is not mapped on extended systems, mainly where heavy atoms are involved [45]. Többens and Kahlenberg proposed a variation of PBE0 with $n=6$ instead of $n=4$ [45], and the results obtained with this proposal show small deviations between experiment and theoretical results for Raman spectra in silicates. One study where the DFT performance of several exchange-correlation functionals was tested on catalytic properties of several $3 d$ and $4 d$ metals shows that the EEX plays an important role in the accuracy of DFT methods. The authors of this report found that $\alpha=0.26$, for $3 d$ metals, and $\alpha=0.34$, for $4 d$ metals, present the lowest RMSDs between experimental and theoretical information [46]. The hybrid B3LYP XC functional has been also used for periodic systems, giving impressive results for the prediction of geometrical parameters, although the estimation of band gaps is not always satisfactory [47, 48].

Heyd, Scuseria, and Ernzerhof (HSE) have reported another way to build hybrid XC functionals, where the contributions of the EEX are divided into long and short ranges. The main purpose of this functional is to improve band gap predictions, which in many cases is reached in a good way $[26,49,50]$, although for some cases this functional must be reparametrized [51]. As support of the HSE XC functionals family, one static dielectric constant, $\epsilon$, has been proposed to estimate the fraction of EEX needed in these functionals [10, 52]. Koller et al. [53] explored this idea, and they propose one scheme where the connection between $\epsilon$ and $\alpha$ is optimized by using experimental band gaps. It was found that there is a linear relationship between the computed band gap and the fraction of EEX used for $\alpha \in[0,1]$. In addition of these proposals, there are reports where the band gap is the main concern, for example Marques et al. reported a work based on physical arguments; unfortunately, such an approach fails when this is applied on systems containing d states [10]. For some metal oxides, Viñes et al. found a linear relationship between one parameter involved in a HSE family of functionals and the dielectric constant [54]. An important conclusion from that work is that there is an infinite number of combinations of the two parameters involved in the HSE XC functionals to obtain the experimental band gap [54]. In another approach, Skone et al. [55] proposed a self-consistent hybrid mixing scheme to determine the exact exchange fraction by computing the optimal dielectric screening, and they found an excellent agreement with experimental band gaps ranging between 0.7 and $21.7 \mathrm{eV}$ and dielectric constants within 1.23 and 15.9.

Alternatively to the DFT-KS approach, the many-body Green's function method, known as the GW approximation $[56,57]$, is directly related to photoemission and inverse photoemission experiments, thereby determining the associated band structure. However, GW calculations are computationally expensive, much more than common DFT-KS methods [11]. Thus, the topic of the correct description of the band gap, in highly correlated electrons, still has a big 
challenge for theoretical and computational methods to provide one reliable methodology predicting band gaps in a reasonable computational cost.

From the discussion given above, we can remark that hybrid exchange-correlation functionals represent an improvement in band gaps with respect to LDA and GGA $\mathrm{XC}$ functionals, giving an alternative to the GW approach [58]. The fraction of EEX to be included in XC hybrid functionals is the main concern in their design, and no always there is a clear way to choice $\alpha$ and which is its effect on some properties [59]. In this report, we give a detailed analysis of the role of this single parameter over structural, mechanical, and electronic properties of some oxides, experimentally well characterized. The oxides tested in this work are $\mathrm{Ti}_{2} \mathrm{O}_{3}, \mathrm{~V}_{2} \mathrm{O}_{3}, \mathrm{Cr}_{2} \mathrm{O}_{3}, \mathrm{Fe}_{2} \mathrm{O}_{3}, \mathrm{MnO}, \mathrm{SnO}_{2}, \mathrm{SiO}_{2}$, and $\mathrm{GeO}_{2}$. All these systems have been studied previously by experimental and theoretical techniques, which will be contrasted with our results. All-electron computations with localized basis set functions are used in this study, where the exact exchange has been incorporated in few studies in a non-systematic way [60-64]. In addition, the role of effective core potentials is not involved in possible differences found for the results reported under the DFT-KS approach, since all-electron calculations are considered for the eight systems.

\section{Computational methods}

All calculations were performed using the periodic ab initio code CRYSTAL14 [65]. Structures, k-points used in the irreducible Brillouin zone and basis set employed for all systems are reported in Table 1; the number of k-points was fixed with the energy convergence for each system. The basis set used for Sn was obtained from the Web site of the Professor Towler [66].

From Eq. (1), six XC functionals were obtained for $\alpha=0.00,0.12,0.18,0.25,0.33$ and 0.50 , where PBE was used as reference for $E_{x}^{\text {approx }}$ and $E_{\mathrm{xc}}^{\text {approx }}$ [70]. The PBE0

Table 1 Entries used to CRYSTAL14

\begin{tabular}{llcl}
\hline System & Structure & $k$-points (IBZ) & Basis set \\
\hline $\mathrm{Fe}_{2} \mathrm{O}_{3}$ & Corundum & 40 & Ref. [60] \\
$\mathrm{MnO}$ & Hard rock salt & 260 & Ref. [64] \\
$\mathrm{Cr}_{2} \mathrm{O}_{3}$ & Corundum & 40 & Ref. [62] \\
$\mathrm{Ti}_{2} \mathrm{O}_{3}$ & Corundum & 294 & Ref. [63] \\
$\mathrm{V}_{2} \mathrm{O}_{3}$ & Corundum & 294 & Ref. [63] \\
& Monoclinic & 294 & Ref. [63] \\
$\mathrm{SiO}_{2}$ & Rutile & 75 & Ref. [67] \\
$\mathrm{GeO}_{2}$ & Rutile & 75 & Ref. [68] \\
$\mathrm{SnO}_{2}$ & Rutile & 75 & Ref. [69] \\
\hline
\end{tabular}

exchange-correlation functional lies in this set $(\alpha=0.25)$ [42] and also PBE0-1/3 functional ( $\alpha=0.33)$ proposed by Cortona and analyzed by Guido [39]. The screened Heyd-Scuseria-Ernzerhof functional, HSE06 [71] and the B1WC [72] functional, which were designed to study ferroelectric oxides, were also considered. The unrestricted Hartree-Fock (UHF) method was used for comparison purposes, which represents a limit case with $\alpha=1.0$ and no correlation contribution. For the $\mathrm{V}_{2} \mathrm{O}_{3}$ system, the PBE XC functional presents convergence problems. However, if a small piece of EEX is incorporated in Eq. (1), then no convergence problems are detected. Thus, for this system, $\alpha=0.06$ was used as the lowest value.

Due to the large amount of theoretical and experimental studies related to hematite, this system is used in this work as reference to analyze in detail the use of XC hybrid functionals in this kind of crystalline systems. Manipulation and visualization of structures were performed with the J-ICE program [73] for all cases.

Full geometry optimizations were carried out for all systems, and gradient components and nuclear displacements were checked for convergence; the RMS gradient was fixed at 0.0003 hartree/bohr and RMS displacement at 0.0012 bohr. The bulk modulus, of each system, was deduced from an elastic constants calculation. They are obtained by a second energy density derivatives with respect to pairs of infinitesimal Eulerian strains, and an automated scheme for the calculation of the elastic tensor has been implemented in the CRYSTAL14 program [74, 75]. The spin-polarized approach was used when it was required, imposing a maximum symmetry. Ferromagnetic (FM) and several antiferromagnetic (AF) states were tested for all electronic structure methods considered in this work. The indirect band gap was obtained from the density of states (DOS) for each system considered in this work.

\section{Results}

\subsection{Magnetic states stability}

For the unit cell of hematite (corundum), all iron atoms have an equivalent octahedral environment, although the chemical environment is different for two pairs of them, as it is appreciated from Fig. 1. Positions of all atoms and cell parameters were relaxed in the optimization process. Thus, two different comparisons can be done for this structure with respect to experimental information: atoms positions and cell parameters.

For temperatures below $960 \mathrm{~K}$, the $\mathrm{Fe}_{2} \mathrm{O}_{3}$ system is predominately an antiferromagnetic (AF) insulator [76, 77], and due to the corundum structure exhibited by this crystal, three different magnetic configurations must be considered 


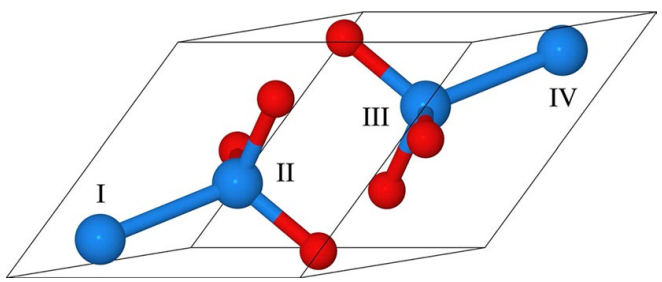

Fig. $1 \mathrm{Fe}_{2} \mathrm{O}_{3}$ primitive cell. Blue spheres represent $\mathrm{Fe}$ and red spheres $\mathrm{O}$ atoms

according to Sandratskii et al. [1]. From Fig. 1, these magnetic configurations (following I, II, III, and IV order) are: (a) $\mathrm{AF}_{1}(+--+)$, (b) $\mathrm{AF}_{2}(+-+-)$, and (c) $\mathrm{AF}_{3}(++--)$; also the ferromagnetic, $\mathrm{FM}(++++)$ configuration was considered in this study. All XC functionals used here were tested to compare the total energy obtained for each configuration; these results are presented in Table 2, where energy differences $\left(\Delta \mathrm{AF}_{i}=\mathrm{AF}_{i}-\mathrm{FM}\right)$ were obtained by using the $\mathrm{FM}$ configuration as reference.

Table 2 gives important information about the $\mathrm{XC}$ functionals tested in this study. First, all of them predict AF configurations more stable than the FM one, which completely agrees with experimental information. Second, the exact exchange has an important impact on $\Delta \mathrm{AF}_{i}$, and it is obtained that the bigger $\alpha$, the smaller $\Delta \mathrm{AF}_{i}$. Therefore, the EEX is quite relevant to study these materials, although this is not the principal component since when the UHF method is used to predict this energy difference, the $\mathrm{AF}_{2}$ configuration is no longer more stable than the FM configuration $\left(\Delta \mathrm{AF}_{2}>0\right)$, and $\Delta \mathrm{AF}_{1}$ and $\Delta \mathrm{AF}_{3}$ are quite small, -0.9 and $-0.2 \mathrm{kcal} / \mathrm{mol}$, respectively, as it has been noted in a previous report [60]. Therefore, the correlation contribution is also important to study this system. It is interesting in Table 2 that PBE0 and HSE06 methods predict same energy differences. In summary, all $\mathrm{XC}$ functionals tested in this work predict $\Delta \mathrm{AF}_{i}<0$, giving smaller values when $\alpha$ is increased. Some of these results are in accord with Rollman et al. [2].

$\mathrm{MnO}$ and $\mathrm{Cr}_{2} \mathrm{O}_{3}$ are antiferromagnetic insulators at low temperatures $[78,79]$. In this study, the $\mathrm{AF}_{2}$ state was confirmed as the most stable phase among all $\mathrm{AF}_{i}$ and $\mathrm{FM}$ states, for both cases, observing similar behavior than that presented for hematite. However, $\mathrm{Ti}_{2} \mathrm{O}_{3}$ and $\mathrm{V}_{2} \mathrm{O}_{3}$ are challenges for DFT. Magnetic properties for $\mathrm{Ti}_{2} \mathrm{O}_{3}$ at low temperatures have been subject of an intensive investigation for both point of views, experimental and theoretical. No general consensus has been achieved [11] to determine whether this material presents AF or diamagnetic behavior at low temperatures [80]. Two $\mathrm{AF}\left(\mathrm{AF}_{1}\right.$ and $\left.\mathrm{AF}_{2}\right)$ spin arrangements have been proposed as more stable [63], and the energy differences between them are quite small. In the present study using PBE0 functional, $\mathrm{AF}_{1}$ is just $0.27 \mathrm{kcal} /$ mol more stable than $\mathrm{AF}_{2}$, which corresponds to a previous report [81]. The diamagnetic state was also tested here, and we obtain that this state is less stable than both AF states. Thus, we will discuss properties of this system considering $\mathrm{AF}_{1}$ and $\mathrm{AF}_{2}$ configurations.

By another side, at low temperatures, $\mathrm{V}_{2} \mathrm{O}_{3}$ becomes an antiferromagnetic insulator with crystal distortions from corundum to monoclinic structure; thus, the metallic phase of $\mathrm{V}_{2} \mathrm{O}_{3}$ has corundum structure and the insulating phase has a monoclinic lattice. In this study, for the corundum structure, the $\mathrm{AF}_{2}$ and $\mathrm{AF}_{3}$ spin configurations are more stable than the ferromagnetic phase, although this one is more stable than the $\mathrm{AF}_{1}$ configuration. The energy difference between the spin arrangements $\mathrm{AF}_{2}$ and $\mathrm{AF}_{3}$ is very small, just $0.64 \mathrm{kcal} / \mathrm{mol}$ by using PBE0. These results are in agreement with those found by Catti et al. [63] when the UHF method is applied over these configurations. In this work, the non-magnetic state has been also considered with broken symmetry. Under this condition, the $\mathrm{AF}_{2}$ state with corundum structure is still the most stable using PBE0. For this reason, the monoclinic and corundum structures will be considered throughout this study.

\subsection{Structural analysis}

From Table 2, it is clear that the $\mathrm{AF}_{1}$ configuration is the most stable for hematite. This system exhibits several structural properties; for this reason, the structure obtained by the methods considered in this article is compared with experimental information. The relative error, in percent, of geometrical parameters between the theoretical estimation
Table 2 Energy difference, per unit formula, between antiferromagnetic $\left(\mathrm{AF}_{i}\right)$ and ferromagnetic (FM) configurations for hematite

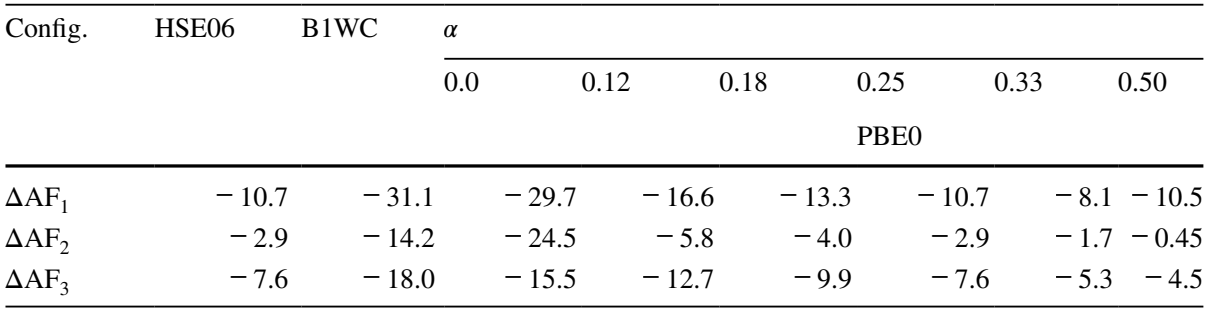

Comparison between exchange-correlation functionals HSE06, B1WC and PBE0 with different exact exchange fractions $(\alpha)$. All quantities are in $\mathrm{kcal} / \mathrm{mol}$ 
for $\mathrm{AF}_{1}$ and the corresponding experimental counterpart [82] is presented in Fig. 2. In this figure, nine geometrical parameters are used for comparison: (1) cell parameter, $c_{0}$; (2) $\mathrm{Fe}-\mathrm{O}^{\prime}$ distance; (3) $\mathrm{Fe}-\mathrm{O}^{\prime \prime}$ distance; (4) $\mathrm{Fe} 1-\mathrm{Fe} 2{ }^{\prime}$ distance; (5) $\mathrm{Fe} 1-\mathrm{Fe} 2^{\prime \prime}$ distance; (6) $\mathrm{Fe} 1-\mathrm{Fe} 2^{\prime \prime \prime}$ distance;
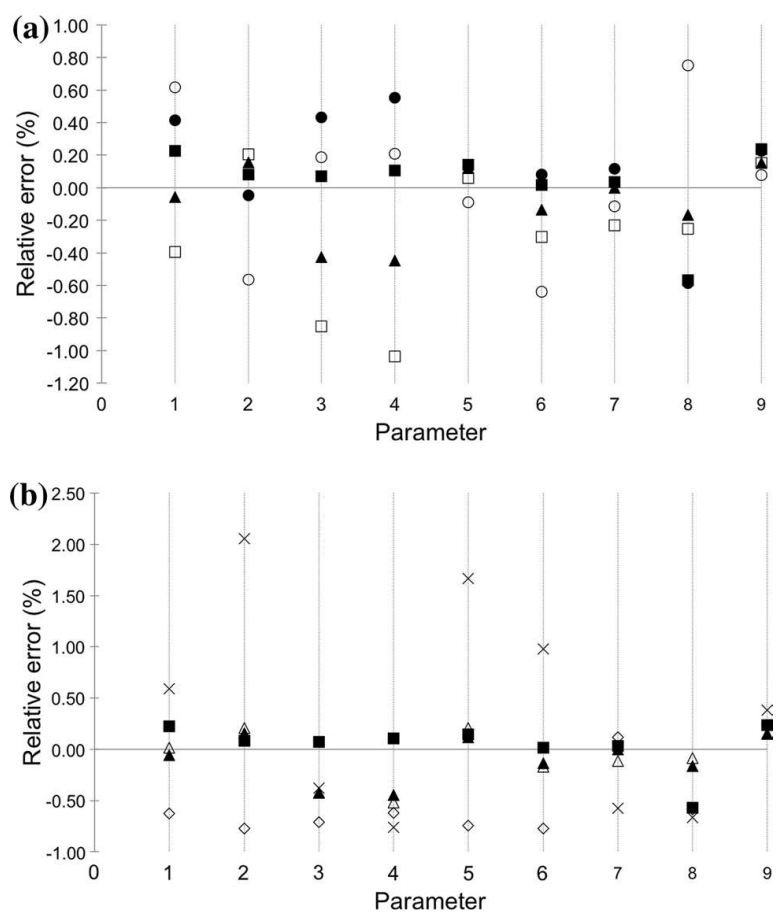

Fig. 2 Relative error of the geometric parameters calculated by the methods: $\alpha=0.00$ (blank circles), $\alpha=0.12$ (solid circles), $\alpha=0.18$ (solid squares), $\alpha=0.25$ (solid triangles), $\alpha=0.33$ (blank squares), UHF (crosses), B1WC (diamonds), HSE06 (blank triangles). (1) Cell parameter, $c_{0}$, (2) $\mathrm{Fe}-\mathrm{O}^{\prime}$ distance, (3) $\mathrm{Fe}-\mathrm{O}^{\prime \prime}$ distance, (4) $\mathrm{Fe} 1-\mathrm{Fe} 2$ ' distances, (5) Fe1-Fe2" distance, (6) Fe1-Fe2'"' distance, (7) Fe1-O$\mathrm{Fe} 2$ ' angle, (8) Fe1-O-Fe2" angle, (9) $\mathrm{Fe} 1-\mathrm{O}-\mathrm{Fe} 2{ }^{\prime \prime \prime}$ angle
(7) $\mathrm{Fe} 1-\mathrm{O}-\mathrm{Fe} 2$ ' angle; (8) $\mathrm{Fe} 1-\mathrm{O}-\mathrm{Fe} 2{ }^{\prime \prime}$ angle; and (9) $\mathrm{Fe} 1-\mathrm{O}-\mathrm{Fe} 2^{\prime \prime \prime}$ angle.

The analysis of Fig. 2 has been separated in two parts. For the first discussion, the impact of the EEX on geometrical parameters is presented in Fig. 2a. Thus, the role of this part of the energy can be appreciated from $\alpha=0.00$ to $\alpha=0.33$ and the cell parameter $\mathrm{c}_{0}$ is clearly reduced when $\alpha$ increases. Although the differences obtained by these $\mathrm{XC}$ functionals are quite small (except for the $\mathrm{Fe} 1-\mathrm{Fe} 2^{\prime}$ distance, no one exceeds $1 \%$ of error), this figure shows that $\alpha=0.18$ gives on average the best prediction for geometrical parameters of hematite. For the second part of the discussion, Fig. 2b, $\alpha=0.18$ and $\alpha=0.25 \mathrm{XC}$ functionals are compared with UHF, B1WC, and HSE06 methods. The first result to be noted is the scale used for the relative error, since in Fig. 2a this difference is not more than $1.2 \%$ and for Fig. 2b, such a difference is enhanced for several methods. In particular, UHF gives the worst prediction followed by the B1WC exchange-correlation functional and this last XC functional was tested because it has been used recently $[83,84]$; however, its performance on the hematite is not good. From this figure, we can conclude that HSE06 functional gives almost same results than those obtained by the PBE0 ( $\alpha=0.25$ ) functional; in addition, within this comparison, the $\alpha=0.18$ functional still gives the best performance.

The effect of $\alpha$ on the structure for all systems considered here is clear from Tables 3 and 4, where we conclude that the exact exchange shrinks the cell parameters of the oxides; the same has been observed before for perovskites with another hybrid functional [89]. For $\mathrm{V}$ and $\mathrm{Ti}$, the spin configurations show the same behavior; the cell parameter is shortened when $\alpha$ is increased, and consequently the PBE functional ( $\alpha=0.00)$ predicts the largest cell parameters.
Table 3 Cell parameter, $\mathrm{c}_{0}$, in Angstroms, predicted by using several fractions $(\alpha)$ of the exact exchange in hybrid functionals

\begin{tabular}{|c|c|c|c|c|c|c|c|}
\hline \multirow[t]{2}{*}{$\alpha$} & \multirow[t]{2}{*}{$\mathrm{Fe}_{2} \mathrm{O}_{3}$} & \multirow[t]{2}{*}{$\mathrm{Cr}_{2} \mathrm{O}_{3}$} & \multirow[t]{2}{*}{$\mathrm{MnO}$} & \multicolumn{2}{|l|}{$\mathrm{Ti}_{2} \mathrm{O}_{3}$} & \multicolumn{2}{|l|}{$\mathrm{V}_{2} \mathrm{O}_{3}$} \\
\hline & & & & $\mathrm{AF}_{1}$ & $\mathrm{AF}_{2}$ & $\mathrm{AF}_{2}$ & $\mathrm{AF}_{3}$ \\
\hline 0.00 & 13.832 & 13.785 & 6.345 & 14.049 & 14.053 & & \\
\hline 0.12 & 13.804 & 13.674 & 6.325 & 13.653 & & 14.004 & \\
\hline 0.18 & 13.773 & 13.640 & 6.316 & 13.605 & & 13.931 & \\
\hline 0.25 & 13.731 & 13.606 & 6.306 & 13.572 & 13.544 & 13.870 & 13.896 \\
\hline 0.33 & 13.686 & 13.566 & 6.294 & 13.548 & & 13.811 & \\
\hline 0.50 & 13.612 & 13.502 & 6.271 & 13.506 & 13.495 & 13.726 & 13.742 \\
\hline Exp. & $13.747[85]$ & 13.578 [85] & 6.286 [86] & 13.610 [87] & & 14.002 [88] & \\
\hline$\alpha_{\mathrm{opt}}$ & 13.812 & 13.663 & 6.309 & 13.580 & & 14.034 & \\
\hline & $(0.5)$ & (0.6) & (0.4) & $(0.2)$ & & $(0.2)$ & \\
\hline
\end{tabular}

In the last row, the cell parameter obtained with $\alpha_{\mathrm{opt}}$ and in parenthesis its corresponding relative error, in percent, with respect to the experimental value. The $\alpha_{\text {opt }}$ is obtained to reproduce the experimental band gap, as it can be seen in Table 6 
Table 4 Cell parameters $\left(\mathrm{c}_{0}\right.$ and $a$ ), in Angstroms, for rutile structures by using several fractions $(\alpha)$ of the exact exchange in hybrid functionals
Table 5 Band gap, $E_{\mathrm{g}}$ in $\mathrm{eV}$, as a function of exact exchange fraction $(\alpha)$ in the PBE0 exchange correlation functional

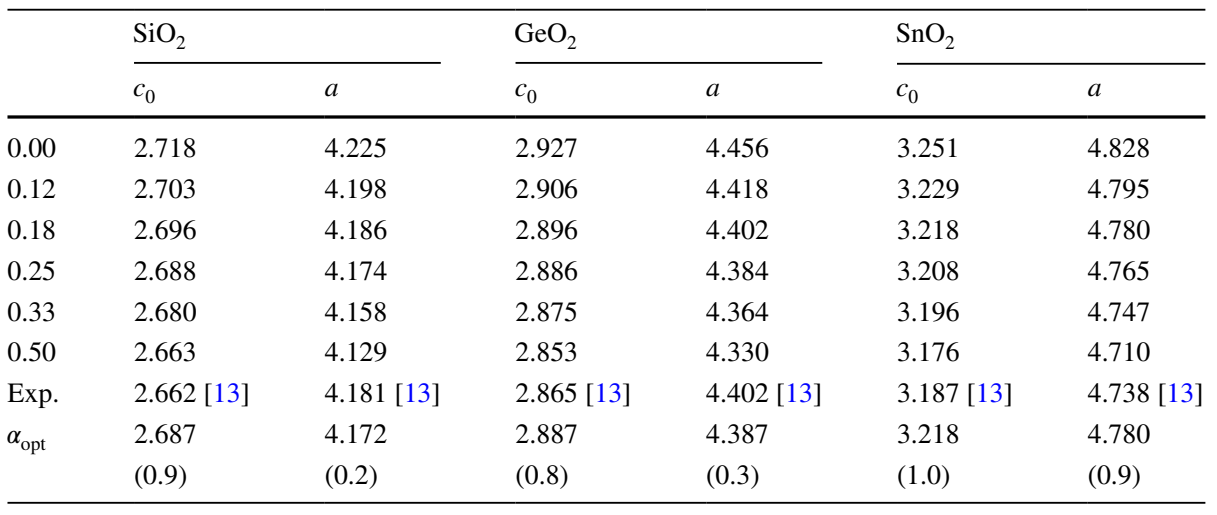

In the last row, the cell parameter obtained with $\alpha_{\mathrm{opt}}$ and in parenthesis its corresponding relative error, in percent, with respect to the experimental value. The $\alpha_{\mathrm{opt}}$ is obtained to reproduce the experimental band gap, as it can be seen in Table 6

\begin{tabular}{lcllllllc}
\hline$\alpha$ & $\mathrm{SiO}_{2}$ & $\mathrm{GeO}_{2}$ & $\mathrm{MnO}$ & $\mathrm{SnO}_{2}$ & $\mathrm{Cr}_{2} \mathrm{O}_{3}$ & $\mathrm{Fe}_{2} \mathrm{O}_{3}$ & $\mathrm{~V}_{2} \mathrm{O}_{3}$ & $\mathrm{Ti}_{2} \mathrm{O}_{3}$ \\
\hline 0.00 & 5.19 & 1.52 & 1.5 & 1.56 & 1.2 & 0.6 & - & - \\
0.12 & 6.88 & 3.05 & 2.9 & 2.88 & 3.3 & 2.3 & 0.97 & 0.37 \\
0.18 & 7.73 & 3.84 & 3.5 & 3.57 & 4.1 & 3.1 & 1.88 & 1.49 \\
0.25 & 8.73 & 4.78 & 4.3 & 4.40 & 5.2 & 4.1 & 2.96 & 2.59 \\
0.33 & 9.88 & 5.88 & 5.3 & 5.37 & 6.4 & 5.3 & 4.24 & 3.85 \\
0.50 & 12.34 & 8.28 & 7.1 & 7.48 & 8.8 & 7.7 & 6.78 & 6.33 \\
Exp. & $8.9[90]$ & $4.68[91]$ & $4.1[92]$ & $3.6[93]$ & $3.4[8]$ & $2.0[3]$ & $0.66[94]$ & $0.11[7]$ \\
& & & & & & $2.7[4]$ & & \\
\hline
\end{tabular}

Experimental values in the last row are given as a reference

\subsection{Band gap}

The role of the EEX over the band gap $E_{\mathrm{g}}$ is reported in Table 5, where available experimental data for this property are also reported for each system, most of them are optical band gaps $\left(\mathrm{SiO}_{2}, \mathrm{GeO}_{2}, \mathrm{SnO}_{2}, \mathrm{MnO}, \mathrm{V}_{2} \mathrm{O}_{3}\right)$, and the others were determined by electrical conductivity, although for hematite optical band gaps values are inside the range referenced here. Experimental band gaps used as references are measured at different temperatures, for $\mathrm{GeO}_{2}$ and $\mathrm{V}_{2} \mathrm{O}_{3}$, the gap is reported at low temperature, and for the others at room temperature or higher; for these last, we expect that for lower temperatures the band gap would diminish.

In Table 5, clearly $\alpha$ increases $E_{\mathrm{g}}$; we must remember that $\alpha=0.00$ corresponds to PBE, and for this functional, $E_{\mathrm{g}}$ presents the lowest value and this is distant from experimental information. In fact, this GGA functional gives $E_{\mathrm{g}}=0.00$ $\mathrm{eV}$ for two systems where this property is no null, $\mathrm{Ti}_{2} \mathrm{O}_{3}$ and $\mathrm{V}_{2} \mathrm{O}_{3}$. In Table 6, parameters of a linear fitting are reported for each oxide. From here, there is no doubt about the linear relationship obtained for $E_{\mathrm{g}}$ versus $\alpha$. Also, for $E_{\mathrm{g}}>1$, the range $\alpha \in[0,0.5]$ gives one value which reproduces the experimental value, which is also in this table as $\alpha_{\text {opt }}$. For
Table 6 Linear fittings for the relationships between the exact exchange $(\alpha)$ and theoretical band gap $\left(E_{\mathrm{g}}, \mathrm{eV}\right)$

\begin{tabular}{|c|c|c|c|c|c|}
\hline & $b$ & $m$ & $R$-squared & $\alpha_{\mathrm{opt}}$ & $\operatorname{Gap}\left(\alpha_{\text {opt }}\right)$ \\
\hline $\mathrm{SiO}_{2}$ & 5.17 & 14.31 & 1.000 & 0.2607 & 8.90 \\
\hline $\mathrm{GeO}_{2}$ & 1.44 & 13.54 & 0.999 & 0.2393 & 4.68 \\
\hline $\mathrm{MnO}$ & 1.49 & 11.30 & 0.999 & 0.2317 & 4.09 \\
\hline $\mathrm{SnO}_{2}$ & 1.48 & 11.87 & 0.999 & 0.1786 & 3.60 \\
\hline $\mathrm{Cr}_{2} \mathrm{O}_{3}$ & 1.34 & 15.04 & 0.998 & 0.1372 & 3.25 \\
\hline $\mathrm{Fe}_{2} \mathrm{O}_{3}$ & 0.58 & 14.20 & 0.999 & 0.1002 & 2.03 \\
\hline $\mathrm{V}_{2} \mathrm{O}_{3}$ & -0.87 & 0.15 & 0.999 & 0.0998 & 0.71 \\
\hline $\mathrm{Ti}_{2} \mathrm{O}_{3}$ & -1.37 & 15.52 & 0.998 & 0.0803 & 0.06 \\
\hline
\end{tabular}

Employing the linear equations, column $\alpha_{\text {opt }}$ presents the adjusted value of $(\alpha)$ to reproduce the experimental band gap; using this value, the gap was calculated, column Gap $\left(\alpha_{\text {opt }}\right)$. Experimental band gaps are in square brackets in Table 5 , all band gaps in $\mathrm{eV}$

$E_{\mathrm{g}}<1$, there are appreciable differences concerning experimental values. Therefore, the best fitting is reached for high band gaps.

For hematite, the prediction obtained by HSE06 and B1WC is 3.3 and $2.7 \mathrm{eV}$, respectively. HSE06 was modified to match the experimental value predicting a band gap 
of $2.0 \mathrm{eV}$ [26] by including EEX in $12 \%$. In addition, the B1WC functional predicts one band gap similar to those values reported experimentally, although this functional has a poor performance predicting structural parameters, as it was discussed above.

Two important conclusions are obtained from the analyzed systems. (1) There is a linear relationship between $E_{\mathrm{g}}$ and the $\alpha$ defined in Eq. (1) to build one-parameter hybrid $\mathrm{XC}$ functionals. (2) One-parameter hybrid XC functionals are able to reproduce experimental band gaps, in particular when $E_{\mathrm{g}}>1 \mathrm{eV}$. These conclusions are in agreement with previous reports [89]. Our work, as previous in this topic, shows that there is not a unique $\alpha$ parameter; each system, depending on the band gap value, will have its own $\alpha$. However, as is it can be seen in Table 6 , for band gaps above 4.0 $\mathrm{eV}$, common PBE0 could give good results, and remember that for this XC functional, $\alpha=0.25$.

There is an additional effect of $\alpha$ on the electronic structure. The density of states (DOS) for spin up associated with $\mathrm{O}_{2 p}$ and $\mathrm{Fe}_{3 d}$ states in hematite is presented for several values of $\alpha$ in Fig. 3; spin-down electrons are not plotted for simplicity. Blue regions correspond to $\mathrm{Fe} d$ orbitals and black lines to $\mathrm{O} p$ orbitals. Large values for $E_{\mathrm{g}}$ are evidenced in this plot when $\alpha$ is increased. Furthermore, all states (occupied and unoccupied) are affected by the EEX. The most important result obtained from DOS is the impact of $\alpha$ on the participation of $\mathrm{Fe}_{3 d}$ and $\mathrm{O}_{2 p}$ orbitals to form the band gap. From our analysis, it is clear that for $\operatorname{PBE}(\alpha=0)$ the $d$ orbitals have the largest contribution, suggesting one $d-d$ band gap. However, when the EEX is present, the $p$ contribution in the conduction band is relevant, predicting a $p-d$ gap, which corresponds to the evidence obtained from experimental information [95]. This example illustrates that one XC functional without contribution of EEX, as $\mathrm{PBE}$, is unable to predict the correct band gap for hematite, in agreement with the well-known deficiency of pure GGA functional in predicting the band gap of solids [96]. This limitation is not just for numerical values; the worst result is that the correct interaction between bands is not described appropriately. This wrong delocalized $\mathrm{Fe}_{3 d}$ electron on conduction band provided by $\mathrm{PBE}$ was corrected by the introduction of a proper Hubbard U parameter for hematite in other study [97]. Moore varied the EEX contribution in the B3LYP exchange-correlation functional from 20 to $10 \%$ and predicted $E_{\mathrm{g}}=1.93 \mathrm{eV}$ for $\mathrm{Fe}_{2} \mathrm{O}_{3}$ [98]. In the same way, Pozun and Henkelman [26] decreased the EEX for the HSE06 exchange-correlation functional and improved the band gap value, as we have discussed above. We remark these reports since HSE06 and B3LYP XC functionals predict one $p-d$ gap $[99,100]$, which is consistent with our results. The present discussion has been around spin up; however, same conclusion can be reached when spin-down DOS is analyzed in the same way.
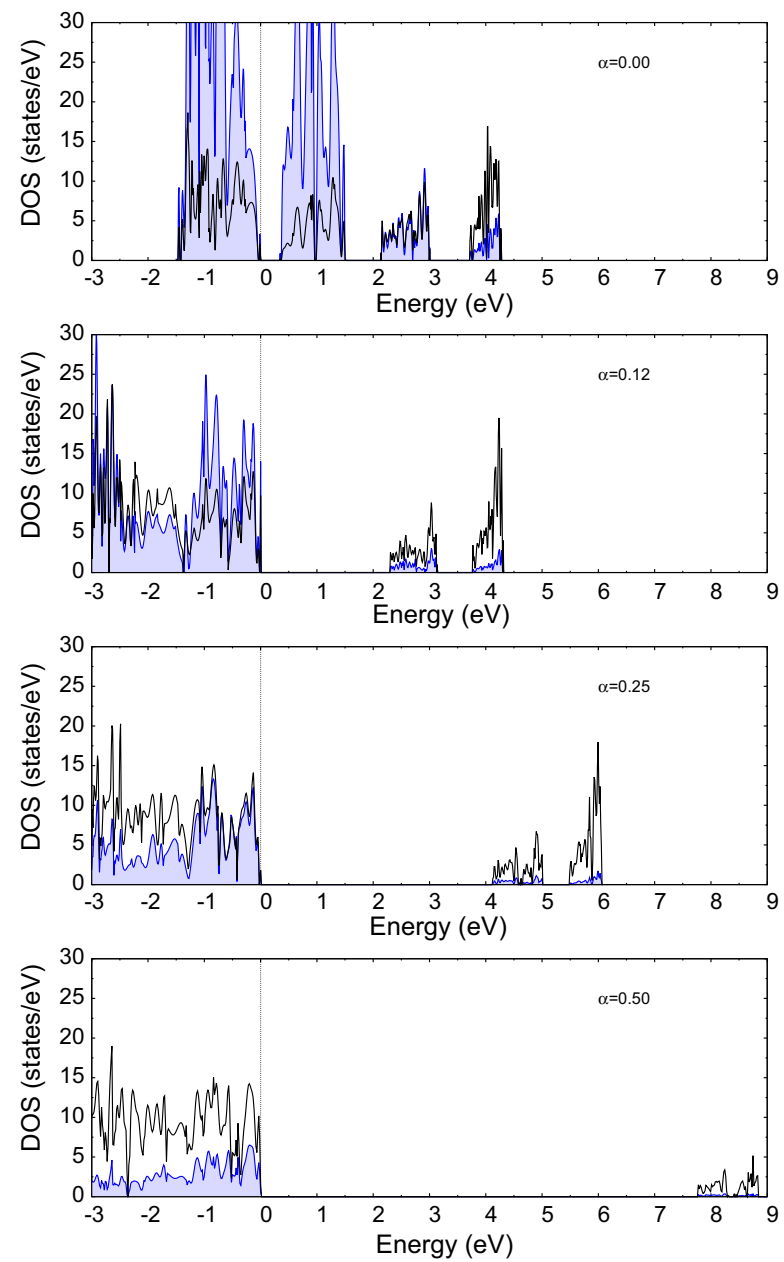

Fig. 3 Projected density of states with spin up for different values of $\alpha$ in hematite. Blue regions, $\mathrm{Fe}_{3 d}$ orbitals. Black line, $\mathrm{O}_{2 p}$ orbitals

For finite systems, the exact exchange has a significant effect on occupied orbitals giving a localized electron density; such an effect is also present in periodic systems. For hematite, in Fig. 4 the electron density is mapped on one plane of the crystal for two values of $\alpha, 0.00$ and 0.50 . From here, the localization of electron density is appreciated mainly on oxygen atoms. Zicovich et al. [101] demonstrated the influence of the Hamiltonian (PBE, PBE0, and HF) over the charge in hematite employing three different population analysis, Mulliken, Hirshfeld-Iterative, and QTAIM. In that study, the same trend was found: PBE predicts small ionic charges. The nonionic character predicted by PBE is expected, due to an artificial overdelocalization of the electron density caused by the extra repulsion due to the self-interaction error in many approximations to XC functionals. By other side, the lack of correlation energy involved in the HF method overemphasizes the ionic character. 


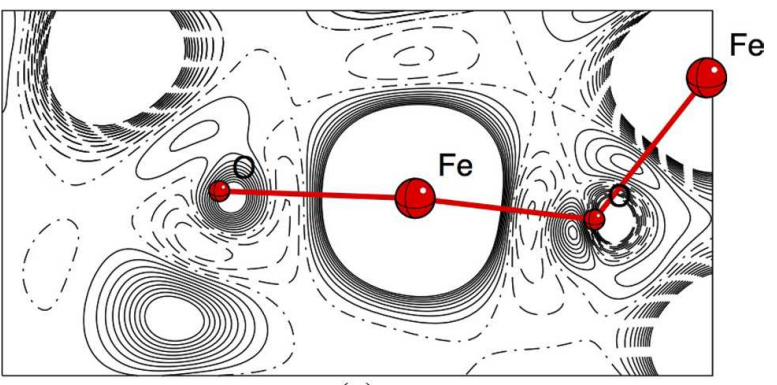

(a)

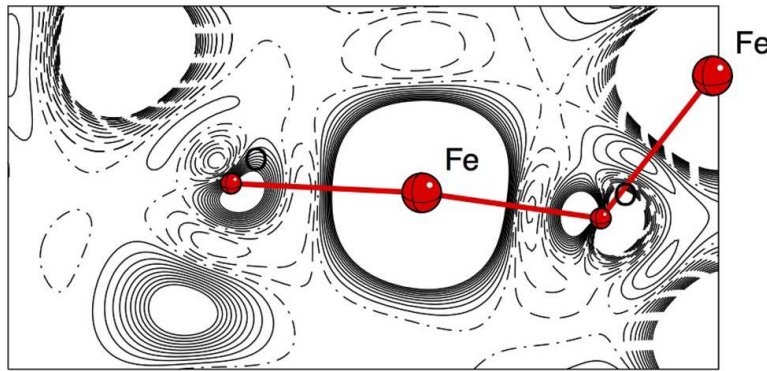

(b)

Fig. 4 Electron density for $\mathrm{Fe}_{2} \mathrm{O}_{3}$. a PBE $(\alpha=0.00)$ and b PBE0 $(\alpha=0.50)$

\subsection{Bulk modulus}

The bulk modulus $\left(B_{0}\right)$ is reported in Table 7 for different values of $\alpha$ in XC functionals represented by Eq. (1). For some cases, experimental data for this property fluctuate in a wide range, indicating that this is an elusive quantity since it depends on the pressure range where this is measured, among other factors as temperature. The bulk modulus in our work is computed by athermal elastic constants; thus, temperature cannot be considered; furthermore, for the systems studied here, there are no experimental bulk moduli at low temperatures. In the comparison, we have considered several experimental values and calculated the maximum error with respect to them. For the systems considered in this article, $B_{0}$ grows up when $\alpha$ is increased, except $\mathrm{Ti}_{2} \mathrm{O}_{3}$ that presents a minimum. As it was also noticed before by Cora et al. [89] for perovskites by using another hybrid functional, "increasing the percentage of HF exchange in the functional, makes the structure more compact and harder." Clearly, the nice linear relationship found for $E_{\mathrm{g}}$ versus $\alpha$ is no longer valid for this property.

For hematite, the results obtained for $\alpha>0$ are within the interval determined by experimental values, with HSE06 (235 GPa) and PBE0 giving almost same predictions. This property has been calculated previously; for example, Rohrbach et al. [123] obtained for this system $B_{0}=173 \mathrm{GPa}$ when PBE is used and $B_{0}=192 \mathrm{GPa}$ for the $\mathrm{PBE}+\mathrm{U}$ approximation. Therefore, the fraction of EEX has an important role on the description of hematite since with PBE and PBE + U approximations, the bulk modulus is out of the range defined by experimental data. For $\alpha=0$, our results do not coincide with those published by Rohrbach et al. [123]; since for this value of $\alpha$, we predict a similar result than these authors for $\mathrm{PBE}+\mathrm{U}$, one possible element for this discrepancy is that in our study we incorporate all electron plus localized functions and they use a pseudopotential approach and maybe the number of plane waves was not enough. The other limit of Eq. 1 is obtained when $\alpha=1$. For this case, Catti et al. by using the UHF method predicted $B_{0}=261 \mathrm{GPa}$ [60], which is a large overestimation for $B_{0}$. No doubt about one-parameter hybrid XC functionals are one important tool to obtain bulk modulus in the range of experimental data for systems where $E_{\mathrm{g}} \neq 0$.

Table 7 Bulk modulus in $\mathrm{GPa}$, as a function of a fraction of the exact exchange $(\alpha)$ in hybrid functionals

\begin{tabular}{lllllllll}
\hline$\alpha$ & $\mathrm{Fe}_{2} \mathrm{O}_{3}$ & $\mathrm{Cr}_{2} \mathrm{O}_{3}$ & $\mathrm{MnO}$ & $\begin{array}{l}\mathrm{Ti}_{2} \mathrm{O}_{3} \\
\mathrm{AF}_{1}\end{array}$ & $\begin{array}{l}\mathrm{V}_{2} \mathrm{O}_{3} \\
\mathrm{AF}_{2}\end{array}$ & $\mathrm{SiO}_{2}$ & $\mathrm{GeO}_{2}$ & $\mathrm{SnO}_{2}$ \\
\hline 0.00 & 190 & 219 & 160 & 221 & & 213 & 279 & 182 \\
0.12 & 217 & 238 & 167 & 206 & 224 & 237 & 299 & 198 \\
0.18 & 227 & 244 & 170 & 200 & 230 & 248 & 308 & 206 \\
0.25 & 236 & 251 & 173 & 209 & 237 & 261 & 318 & 213 \\
0.33 & 244 & 259 & 176 & 220 & 243 & 275 & 330 & 224 \\
0.50 & 262 & 273 & 182 & 218 & 255 & 300 & 354 & 242 \\
Exp. & $207-258$ & $231-259$ & $147-162$ & 206,237 & $175-214$ & $261,308,313$ & 267,251 & $203,205,212$ \\
& {$[82,102-106]$} & {$[82,102,107]$} & {$[108-111]$} & {$[112,113]$} & {$[82,102,114]$} & {$[115-117]$} & {$[118,119]$} & {$[120-122]$} \\
$\alpha$ & 213 & 240 & 172 & 206 & 221 & 263 & 316 & 206 \\
& $(17)$ & $(7)$ & $(17)$ & $(13)$ & $(26)$ & $(16)$ & $(26)$ & $(3)$ \\
\hline
\end{tabular}

In the last row, the bulk modulus obtained with $\alpha_{\mathrm{opt}}$ and in parenthesis its maximum percentage of relative error with respect to the experimental value. The $\alpha_{\mathrm{opt}}$ is obtained to reproduce the experimental band gap, as it can be seen in Table 6 


\subsection{The parameter $\alpha_{\text {opt }}$ to predict cell parameters and bulk modulus}

The $\alpha_{\text {opt }}$ obtained for each system from the linear relation $E_{\mathrm{g}}$ versus $\alpha$ was used to explore its effects over structural parameters and bulk modulus. A six-order Lagrange polynomial was used to interpolate the corresponding results reported in Tables 3, 4, and 7. For structural parameters, a small deviation appears between our predictions and the corresponding experimental data, since the biggest error is $1 \%$. These deviations are reported in the last row in parenthesis of each table mentioned above. For bulk modulus, the use of $\alpha_{\text {opt }}$ in the interpolation approach gives results close to those reported experimentally. Therefore, the linear relationship between the band gap and a fraction of the exact exchange provides an $\alpha_{\text {opt }}$ that allows to predict cell parameters and bulk modulus close to experimental values.

\section{Conclusions}

We have shown that fractions of the exact exchange play an essential role in the prediction of properties of oxides of different nature. According to previous studies for other systems, there is a linear relationship between the band gap and the fraction of exact exchange included in oneparameter hybrid exchange-correlation functionals. For systems with a band gap greater than $1.0 \mathrm{eV}$, the linear relationship allows theoretical results to match the experimental counterpart. From this optimum fraction of EXX $\left(\alpha_{\text {opt }}\right)$, cell parameters and bulk modulus predictions show small deviations of experimental data. This article and other published previously show that there is no an universal hybrid functional to describe correctly all properties of metal or metalloid oxides.

Acknowledgements This article is dedicated to the memory of our friend Claudio M. Zicovich-Wilson, who opened a new perspective of the solid state chemistry in México. We really miss him for his compromise with the academy and with his friends. We thank the Laboratorio de Supercómputo y Visualización en Paralelo at the Universidad Autónoma Metropolitana-Iztapalapa for access to their computer facilities. We also thank CONACYT, México, for financial support throughout project 154784 and the Red Temática de Fisicoquímica Teórica. M. Rivera-Almazo thanks CONACYT for the scholarship 633579.

\section{References}

1. Sandratskii LM, Uhl M, Kübler J (1996) Band theory for electronic and magnetic properties of $\alpha-\mathrm{Fe}_{2} \mathrm{O}_{3}$. J Phys Condens Matter 8:983-989

2. Rollmann G, Rohrbach A, Entel P, Hafner J (2004) First-principles calculation of the structure and magnetic phases of hematite. Phys Rev B 69:165107
3. Mochizuki S (1977) Electrical conductivity of $\alpha-\mathrm{Fe}_{2} \mathrm{O}_{3}$. Phys Status Solidi A 41:591-594

4. Glasscock JA, Barnes PRF, Plumb IC, Bendavid A, Martin PJ (2008) Structural, optical and electrical properties of undoped polycrystalline hematite thin films produced using filtered arc deposition. Thin Solid Films 516:1716-1724

5. Mattheiss. LF (1996) Electronic structure of rhombohedral $\mathrm{Ti}_{2} \mathrm{O}_{3}$. J Phys Condens Matter 8:5987-5995

6. Imada M, Fujimori A, Tokura Y (1998) Metal-insulator transitions. Rev Mod Phys 70:1039-1263

7. Shin SH, Chandrashekhar GV, Loehman RE, Honig JM (1973) Thermoelectric effects in pure and $\mathrm{V}$-doped $\mathrm{Ti}_{2} \mathrm{O}_{3}$ single crystals. Phys Rev B 8:1364-1372

8. Adler D (1968) Insulating and metallic states in transition metal oxides. In: Seitz F (ed) Solid state physics, vol 21. Academic Press, New York, pp 1-115

9. Gillen R, Robertson J (2013) Accurate screened exchange band structures for the transition metal monoxides $\mathrm{MnO}, \mathrm{FeO}, \mathrm{CoO}$ and NiO. J Phys Condens Matter 25:165502

10. Marques MAL, Vidal J, Oliveira MJT, Reining L, Botti S (2011) Density-based mixing parameter for hybrid functionals. Phys Rev B 83:035119

11. Gerosa M, Bottani CE, Caramella L, Onida G, di Valentin C, Pacchioni G (2015) Electronic structure and phase stability of oxide semiconductors: Performance of dielectric-dependent hybrid functional DFT, benchmarked against GW band structure calculations and experiments. Phys Rev B 91:155201

12. Ravindra NM, Weeks RA, Kinser DL (1987) Optical-properties of $\mathrm{GeO}_{2}$. Phys Rev B 36:6132-6134

13. Yamanaka T, Kurashima R, Mimaki J (2000) X-ray diffraction study of bond character of rutile-type $\mathrm{SiO}_{2}, \mathrm{GeO}_{2}$ and $\mathrm{SnO}_{2}$. Z Kristallogr 215:424-428

14. Mimaki J, Tsuchiya T, Yamanaka T (2000) The bond character of rutile type $\mathrm{SiO}_{2}, \mathrm{GeO}_{2}$ and $\mathrm{SnO}_{2}$ investigated by molecular orbital calculation. Z Kristallogr 215:419-423

15. Micoulaut M, Cormier L, Henderson GS (2006) The structure of amorphous, crystalline and liquid $\mathrm{GeO}_{2}$. J Phys Condes Matter 18:R753-R784

16. Sevik C, Bulutay C (2007) Theoretical study of the insulating oxides and nitrides: $\mathrm{SiO}_{2}, \mathrm{GeO}_{2}, \mathrm{Al}_{2} \mathrm{O}_{3}, \mathrm{Si}_{3} \mathrm{~N}_{4}$, and $\mathrm{Ge}_{3} \mathrm{~N}_{4}$. J Mater Sci 42:6555-6565

17. Shaposhnikov AV, Perevalov TV, Gritsenko VA, Cheng CH, Chin A (2012) Mechanism of $\mathrm{GeO}_{2}$ resistive switching based on the multi-phonon assisted tunneling between traps. Appl Phys Lett 100:243506

18. Singh H, Singh M, Kumar S, Kashyap MK (2011) Full potential calculation of electronic properties of rutile $\mathrm{RO}_{2}(\mathrm{R}=\mathrm{Si}$, $\mathrm{Ge}, \mathrm{Sn}$ and $\mathrm{Pb}$ ) compounds via modified Becke Johnson potential. Phys B Condens Matter 406:3825-3830

19. Liu QJ, Liu ZT (2014) Structural, elastic, and mechanical properties of germanium dioxide from first-principles calculations. Mater Sci Semicond Process 27:765-776

20. Anisimov VI, Aryasetiawan F, Lichtenstein AI (1997) Firstprinciples calculations of the electronic structure and spectra of strongly correlated systems: the LDA + U method. J Phys Condens Matter 9:767-808

21. Dudarev SL, Botton GA, Savrasov SY, Humphreys CJ, Sutton AP (1998) Electron-energy-loss spectra and the structural stability of nickel oxide: an LSDA + U study. Phys Rev B 57:1505-1509

22. Dudarev SL, Botton GA, Savrasov SY, Szotek Z, Temmerman WM, Sutton AP (1998) Electronic structure and elastic properties of strongly correlated metal oxides from first principles: LSDA + U, SIC-LSDA and EELS study of $\mathrm{UO}_{2}$ and NiO. Phys Status Solidi A 166:429-443 
23. Rohrbach A, Hafner J, Kresse G (2003) Electronic correlation effects in transition-metal sulfides. J Phys Condens Matter 15:979-996

24. Wang L, Maxisch T, Ceder G (2006) Oxidation energies of transition metal oxides within the GGA + U framework. Phys Rev B 73:195107

25. Wang YC, Chen ZH, Jiang H (2016) The local projection in the density functional theory plus $U$ approach: a critical assessment. J Chem Phys 144:144106

26. Pozun ZD, Henkelman G (2011) Hybrid density functional theory band structure engineering in hematite. J Chem Phys 134:224706

27. Jiang H, Gomez-Abal RI, Rinke P, Scheffler M (2010) Firstprinciples modeling of localized $d$ states with the GW@LDA + U approach. Phys Rev B 82:045108

28. Perdew JP, Levy M (1983) Physical content of the exact KohnSham orbital energies: band gaps and derivative discontinuities. Phys Rev Lett 51:1884-1887

29. Sham LJ, Schlüter M (1985) Density-functional theory of the band gap. Phys Rev B 32:3883-3889

30. Görling A (2015) Exchange-correlation potentials with proper discontinuities for physically meaningful Kohn-Sham eigenvalues and band structures. Phys Rev B 91:245120

31. Cencek W, Szalewicz K (2013) On asymptotic behavior of density functional theory. J Chem Phys 139:024104

32. Garza J, Nichols JA, Dixon DA (2000) The optimized effective potential and the self-interaction correction in density functional theory: application to molecules. J Chem Phys 112:7880-7890

33. Kummel S, Kronik L (2008) Orbital-dependent density functionals: theory and applications. Rev Mod Phys 80:3-60

34. Gopal P, Fornari M, Curtarolo S, Agapito LA, Liyanage LSI, Nardelli MB (2015) Improved predictions of the physical properties of $\mathrm{Zn}$ - and $\mathrm{Cd}$-based wide band-gap semiconductors: a validation of the ACBN0 functional. Phys Rev B 91:245202

35. Unal H, Gunceler D, Gulseren O, Ellialtioglu S, Mete E (2015) Hybrid functional calculated optical and electronic structures of thin anatase $\mathrm{TiO}_{2}$ nanowires with organic dye adsorbates. Appl Surf Sci 354:437-442

36. Seo DH, Urban A, Ceder G (2015) Calibrating transition-metal energy levels and oxygen bands in first-principles calculations: accurate prediction of redox potentials and charge transfer in lithium transition-metal oxides. Phys Rev B 92:115118

37. Burke K, Ernzerhof M, Perdew JP (1997) The adiabatic connection method: a non-empirical hybrid. Chem Phys Lett 265:115-120

38. Cortona P (2012) Theoretical mixing coefficients for hybrid functionals. J Chem Phys 136:086101

39. Guido CA, Brémond E, Adamo C, Cortona P (2013) Communication: one third: a new recipe for the PBE0 paradigm. J Chem Phys 138:021104

40. Tran F, Blaha P, Schwarz K, Novák P (2006) Hybrid exchangecorrelation energy functionals for strongly correlated electrons: applications to transition-metal monoxides. Phys Rev B 74:155108

41. Perdew JP, Ernzerhof M, Burke K (1996) Rationale for mixing exact exchange with density functional approximations. J Chem Phys 105:9982-9985

42. Adamo C, Barone V (1999) Toward reliable density functional methods without adjustable parameters: the PBE0 model. J Chem Phys 110:6158-6170

43. Demichelis R, Civalleri B, Ferrabone M, Dovesi R (2010) On the performance of eleven DFT functionals in the description of the vibrational properties of aluminosilicates. Int J Quantum Chem 110:406-415

44. De La Pierre M, Orlando R, Maschio L, Doll K, Ugliengo P, Dovesi R (2011) Performance of six functionals (LDA, PBE,
PBESOL, B3LYP, PBE0, and WC1LYP) in the simulation of vibrational and dielectric properties of crystalline compounds. The case of forsterite $\mathrm{Mg}_{2} \mathrm{SiO}_{4}$. J Comput Chem 32:1775-1784

45. Többens DM, Kahlenberg V (2011) Improved DFT calculation of Raman spectra of silicates. Vib Spectrosc 56:265-272

46. Liu C, Peterson C, Wilson AK (2013) C-O bond cleavage of dimethyl ether by transition metal ions: a systematic study on catalytic properties of metals and performance of DFT functionals. J Phys Chem A 117:5140-5148

47. Martínez-Casado R, Chen VHY, Mallia G, Harrison NM (2016) A hybrid-exchange density functional study of the bonding and electronic structure in bulk CuFeS 2 . J Chem Phys 144:184702

48. De Fusco GC, Pisani L, Montanari B, Harrison NM (2009) Density functional study of the magnetic coupling in V ( TCNE $)_{2}$. Phys Rev B 79:085201

49. Brothers EN, Izmaylov AF, Normand JO, Barone V, Scuseria GE (2008) Accurate solid-state band gaps via screened hybrid electronic structure calculations. J Chem Phys 129:011102

50. Garza AJ, Scuseria GE (2016) Predicting band gaps with hybrid density functionals. J Phys Chem Lett 7:4165-4170

51. Iori F, Gatti M, Rubio A (2012) Role of nonlocal exchange in the electronic structure of correlated oxides. Phys Rev B 85:115129

52. Shimazaki T, Asai Y (2008) Band structure calculations based on screened Fock exchange method. Chem Phys Lett 466:91-94

53. Koller D, Blaha P, Tran F (2013) Hybrid functionals for solids with an optimized Hartree-Fock mixing parameter. J Phys Condens Matter 25:435503

54. Viñes F, Lamiel-García O, Chul-Ko K, Yong-Lee J, Illas F (2017) Systematic study of the effect of HSE functional internal parameters on the electronic structure and band gap of a representative set of metal oxides. J Comput Chem 38:781-789

55. Skone JH, Govoni M, Galli G (2014) Self-consistent hybrid functional for condensed systems. Phys Rev B 89:195112

56. Hedin L (1965) New method for calculating the one-particle Green's function with application to the electron-gas problem. Phys Rev 139:A796

57. Onida G, Reining L, Rubio A (2002) Electronic excitations: density-functional versus many-body Green's-function approaches. Rev Mod Phys 74:601-659

58. Alkauskas A, Broqvist P, Devynck F, Pasquarello A (2008) Band offsets at semiconductor-oxide interfaces from hybrid densityfunctional calculations. Phys Rev Lett 101:106802

59. Alkauskas A, Broqvist P, Pasquarello A (2011) Defect levels through hybrid density functionals: insights and applications. Phys Status Solidi B 248:775-789

60. Catti M, Valerio G, Dovesi R (1995) Theoretical study of electronic, magnetic, and structural properties of $\alpha$-Fe ${ }_{2} \mathrm{O}_{3}$ (hematite). Phys Rev B 51:7441-7450

61. Catti M, Sandrone G, Valerio G, Dovesi R (1996) Electronic, magnetic and crystal structure of $\mathrm{Cr}_{2} \mathrm{O}_{3}$ by theoretical methods. J Phys Chem Solids 57:1735-1741

62. Catti M, Sandrone G (1997) Ab initio study of corundum-like $\mathrm{Me}_{2} \mathrm{O}_{3}$ oxides $(\mathrm{Me}=\mathrm{Ti}, \mathrm{V}, \mathrm{Cr}, \mathrm{Fe} \mathrm{Co}, \mathrm{Ni})$. Faraday Discuss 106:189-203

63. Catti M, Sandrone G, Dovesi R (1997) Periodic unrestricted Hartree-Fock study of corundumlike $\mathrm{Ti}_{2} \mathrm{O}_{3}$ and $\mathrm{V}_{2} \mathrm{O}_{3}$. Phys Rev B 55:16122-16131

64. Towler MD, Allan NL, Harrison NM, Saunders VR, Mackrodt WC, Aprà E (1994) Ab initio study of MnO and NiO. Phys Rev B 50:5041-5041

65. Dovesi R, Orlando R, Erba A, Zicovich-Wilson CM, Civalleri B, Casassa S, Maschio L, Ferrabone M, De La Pierre M, D’Arco P et al (2014) Crystal14: a program for the ab initio investigation of crystalline solids. Int J Quantum Chem 114:1287-1317

66. Towler M (2017) Crystal resources page. http://www.tcm.phy. cam.ac.uk/mdt26/crystal.html. Accessed on Oct 2017 
67. D'Arco P, Sandrone G, Dovesi R, Orlando R, Saunders VR (1993) A quantum-mechanical study of the perovskite structure type of $\mathrm{MgSiO}_{3}$. Phys Chem Miner 20:407-414

68. CRYSTAL Theoretical Chemistry Group (2017) Basis sets library. http://www.crystal.unito.it/basis-sets.php. Accessed 10 Oct 2017

69. Corà F (2005) The performance of hybrid density functionals in solid state chemistry: the case of $\mathrm{BaTiO}_{3}$. Mol Phys 103:2483-2496

70. Perdew JP, Burke K, Ernzerhof M (1996) Generalized gradient approximation made simple. Phys Rev Lett 77:3865-3868

71. Heyd J, Scuseria GE, Ernzerhof M (2006) Erratum: hybrid functionals based on a screened coulomb potential [J. Chem. Phys. 118, 8207 (2003)]. J Chem Phys 124:219906

72. Bilc DI, Orlando R, Shaltaf R, Rignanese GM, Íniguez J, Ghosez P (2008) Hybrid exchange-correlation functional for accurate prediction of the electronic and structural properties of ferroelectric oxides. Phys Rev B 77:165107

73. Canepa P, Hanson RM, Ugliengo P, Alfredsson M (2011) J-ICE: a new Jmol interface for handling and visualizing crystallographic and electronic properties. J Appl Crystallogr 44:225-229

74. Perger WF, Criswell J, Civalleri B, Dovesi R (2009) Ab-initio calculation of elastic constants of crystalline systems with the CRYSTAL code. Comput Phys Commun 180:1753-1759

75. Erba A, Mahmoud A, Orlando R, Dovesi R (2014) Elastic properties of six silicate garnet end members from accurate ab initio simulations. Phys Chem Miner 41:151-160

76. Freier S, Greenshpan M, Hillman P, Shechter H (1962) The antiferromagnetic Curie point in $\alpha-\mathrm{Fe}_{2} \mathrm{O}_{3}$. Phys Lett 2:191-192

77. Lielmezs J, Chaklader ACD (1965) Reversible thermal effect in $\alpha-\mathrm{Fe}_{2} \mathrm{O}_{3}$ at $690^{\circ} \pm 5^{\circ} \mathrm{C}$. J Appl Phys 36:866

78. Fender BEF, Jacobson AJ, Wedgwood FA (1968) Covalency parameters in MnO, $\alpha-\mathrm{MnS}$, and NiO. J Chem Phys 48:990-994

79. Brockhouse $\mathrm{BN}$ (1953) Antiferromagnetic structure in $\mathrm{Cr}_{2} \mathrm{O}_{3}$. J Chem Phys 21:961-962

80. Guo Y, Clark SJ, Robertson J (2012) Electronic and magnetic properties of $\mathrm{Ti}_{2} \mathrm{O}_{3}, \mathrm{Cr}_{2} \mathrm{O}_{3}$, and $\mathrm{Fe}_{2} \mathrm{O}_{3}$ calculated by the screened exchange hybrid density functional. J Phys Condens Matter 24:325504

81. Hu Z, Metiu H (2011) Choice of U for DFT + U calculations for titanium oxides. J Phys Chem C 115:5841-5845

82. Finger LW, Hazen RM (1980) Crystal structure and isothermal compression of $\mathrm{Fe}_{2} \mathrm{O}_{3}, \mathrm{Cr}_{2} \mathrm{O}_{3}$, and $\mathrm{V}_{2} \mathrm{O}_{3}$ to $50 \mathrm{kbars}$. J Appl Phys 51:5362-5367

83. Lemal S, Nguyen N, de Boor J, Ghosez P, Varignon J, Klobes B, Hermann RP, Verstraete MJ (2015) Thermoelectric properties of the unfilled skutterudite $\mathrm{FeSb}_{3}$ from first principles and Seebeck local probes. Phys Rev B 92:205204

84. Seo YS, Ahn JS (2013) Pressure dependence of the phonon spectrum in $\mathrm{BaTiO}_{3}$ polytypes studied by ab initio calculations. Phys Rev B 88:014114

85. Belokoneva EL, Shcherbakova YK (2003) Electron density in synthetic escolaite $\mathrm{Cr}_{2} \mathrm{O}_{3}$ with a corundum structure and its relation to antiferromagnetic properties. Russ J Inorg Chem 48:861-869

86. Wyckoff RWG (1963) Crystal structures. Wiley, New York

87. Rice CE, Robinson WR (1977) High-temperature crystal chemistry of $\mathrm{Ti}_{2} \mathrm{O}_{3}$ : structural changes accompanying the semiconductor-metal transition. Acta Crystallogr B 33:1342-1348

88. Newnham EE, de Haan YM (1962) Refinement of the $\alpha \mathrm{Al}_{2} \mathrm{O}_{3}$, $\mathrm{Ti}_{2} \mathrm{O}_{3}, \mathrm{~V}_{2} \mathrm{O}_{3}$ and $\mathrm{Cr}_{2} \mathrm{O}_{3}$ structures. Z Kristallogr New Cryst Struct 117:235-237

89. Corà F, Alfredsson M, Mallia G, Middlemiss DS, Mackrodt WC, Dovesi R, Orlando R (2004) The performance of hybrid density functionals in solid state chemistry. Struct Bond 113:171-232
90. Maj S (1988) Energy-gap and density in $\mathrm{SiO}_{2}$ polymorphs. Phys Chem Miner 15:271-273

91. Stapelbroek M, Evans BD (1978) Exciton structure in UVabsorption edge of tetragonal $\mathrm{GeO}_{2}$. Solid State Commun 25:959-962

92. Kurmaev EZ, Wilks RG, Moewes A, Finkelstein LD, Shamin SN, Kuneš J (2008) Oxygen X-ray emission and absorption spectra as a probe of the electronic structure of strongly correlated oxides. Phys Rev B 77:165127

93. Batzill M, Diebold U (2005) The surface and materials science of tin oxide. Prog Surf Sci 79:47-154

94. Thomas GA, Rapkine DH, Carter SA, Millis AJ, Rosenbaum TF, Metcalf P, Honig JM (1994) Observation of the gap and kinetic energy in a correlated insulator. Phys Rev Lett 73:1529-1532

95. Uozumi T, Okada K, Kotani A (1996) Theory of photoemission spectra for $\mathrm{M}_{2} \mathrm{O}_{3}(\mathrm{M}=\mathrm{Ti}, \mathrm{V}, \mathrm{Cr}, \mathrm{Mn}, \mathrm{Fe})$ compounds. J Electron Spectros Relat Phenom 78:103-106

96. Perdew JP, Yang W, Burke K, Yanga Z, Gross EKU, Scheffler M, Scuseriaj GE, Henderson TM, Zhang IY, Ruzsinszky A, Peng H, Sun J, Trushin E, Görling A (2017) Understanding band gaps of solids in generalized Kohn-Sham theory. PNAS 114:2801-2806

97. Meng Y, Liu XW, Huo CF, Guo WP, Cao DB, Peng Q, Dearden A, Gonze X, Yang Y, Wang J et al (2016) When density functional approximations meet iron oxides. J Chem Theory Comput 12:5132-5144

98. Moore EA (2007) First-principles study of the mixed oxide $\alpha$ -FeCrO 3 . Phys Rev B 76:195107

99. Wilson NC, Russo SP (2009) Hybrid density functional theory study of the high-pressure polymorphs of $\alpha$-Fe ${ }_{2} \mathrm{O}_{3}$ hematite. Phys Rev B 79:094113

100. Cramer CJ, Truhlar DG (2009) Density functional theory for transition metals and transition metal chemistry. Phys Chem Chem Phys 11:10757-10816

101. Zicovich-Wilson CM, Hô M, Navarrete-López AM, Casassa S (2016) Hirshfeld-I charges in linear combination of atomic orbitals periodic calculations. Theor Chem Acc 135:1-13

102. Sato Y, Akimoto S (1979) Hydrostatic compression of four corundum-type compounds: $\alpha-\mathrm{Al}_{2} \mathrm{O}_{3}, \mathrm{~V}_{2} \mathrm{O}_{3}, \mathrm{Cr}_{2} \mathrm{O}_{3}$, and $\alpha$ $-\mathrm{Fe}_{2} \mathrm{O}_{3}$. J Appl Phys 50:5285-5291

103. Schouwink P, Dubrovinsky L, Glazyrin K, Merlini M, Hanfland M, Pippinger T, Miletich R (2011) High-pressure structural behavior of $\alpha$-Fe ${ }_{2} \mathrm{O}_{3}$ studied by single-crystal X-ray diffraction and synchrotron radiation up to $25 \mathrm{GPa}$. Am Mineral 96:1781-1786

104. Sumino Y, Anderson OL (1984) Elastic constants of minerals. In: Carmichael RS (ed) Handbook of physical properties of rocks, vol 3. CRC Press, Boca Raton, pp 39-138

105. Olsen JS, Cousins CSG, Gerward L, Jhans HT, Sheldon BJ (1991) A study of the crystal structure of $\mathrm{Fe}_{2} \mathrm{O}_{3}$ in the pressure range up to $65 \mathrm{GPa}$ using synchrotron radiation. Phys Scr 43:327

106. Rozenberg GK, Dubrovinsky LS, Pasternak MP, Naaman O, Le Bihan T, Ahuja R (2002) High-pressure structural studies of hematite $\mathrm{Fe}_{2} \mathrm{O}_{3}$. Phys Rev B 65:064112

107. Mougin J, Le Bihan T, Lucazeau G (2001) High-pressure study of $\mathrm{Cr}_{2} \mathrm{O}_{3}$ obtained by high-temperature oxidation by X-ray diffraction and Raman spectroscopy. J Phys Chem Solids 62:553-563

108. Ohnishi S, Mizutani H (1978) Crystal field effect on bulk moduli of transition metal oxides. J Geophys Res Solid Earth 83:1852-1856

109. Noguchi Y, Kusaba K, Fukuoka K, Syono Y (1996) Shockinduced phase transition of $\mathrm{MnO}$ around $90 \mathrm{GPa}$. Geophys Res Lett 23:1469-1472

110. Makino Y, Miyake S (2000) Estimation of bulk moduli of compounds by empirical relations between bulk modulus and interatomic distance. J Alloys Compd 313:235-241 
111. Jeanloz R, Rudy A (1987) Static compression of MnO manganosite to $60 \mathrm{GPa}$. J Geophys Res Solid Earth 92:11433-11436

112. Nishio-Hamane D, Katagiri M, Niwa K, Sano-Furukawa A, Okada T, Yagi T (2009) A new high-pressure polymorph of $\mathrm{Ti}_{2} \mathrm{O}_{3}$ : implication for high-pressure phase transition in sesquioxides. High Press Res 29:379-388

113. Ovsyannikov SV, Wu X, Shchennikov VV, Karkin AE, Dubrovinskaia N, Garbarino G, Dubrovinsky L (2010) Structural stability of a golden semiconducting orthorhombic polymorph of $\mathrm{Ti}_{2} \mathrm{O}_{3}$ under high pressures and high temperatures. J Phys Condens Matter 22:375402

114. McWhan DB, Remeika JP (1970) Metal-insulator transition in $\left(\mathrm{V}_{1-\mathrm{x}} \mathrm{Cr}_{\mathrm{x}}\right)_{2} \mathrm{O}_{3}$. Phys Rev B 2:3734-3750

115. Demuth T, Jeanvoine Y, Hafner J, Angyan JG (1999) Polymorphism in silica studied in the local density and generalized-gradient approximations. J Phys Condens Matter 11:3833-3874

116. Weidner DJ, Bass JD, Ringwood AE, Sinclair W (1982) The single-crystal elastic-moduli of stishovite. J Geophys Res 87:4740-4746

117. Panero WR, Benedetti LR, Jeanloz R (2003) Equation of state of stishovite and interpretation of $\mathrm{SiO}_{2}$ shock-compression data. $\mathrm{J}$ Geophys Res Solid Earth 108:ECV5-1
118. Christie DM, Chelikowsky JR (2000) Electronic and structural properties of germania polymorphs. Phys Rev B 62:14703-14711

119. Wang H, Simmons G (1973) Elasticity of some mantle crystalstructures: 2. Rutile $\mathrm{GeO}_{2}$. J Geophys Res 78:1262-1273

120. Liebermann RC (1973) Elastic properties of polycrystalline $\mathrm{SnO}_{2}$ and $\mathrm{GeO}_{2}$ : comparison with stishovite and rutile data. Phys Earth Planet Inter 7:461-465

121. Haines J, Léger JM (1997) X-ray diffraction study of the phase transitions and structural evolution of tin dioxide at high pressure: relationships between structure types and implications for other rutile-type dioxides. Phys Rev B 55:11144-11154

122. Chang E, Graham EK (1975) Elastic-constants of cassiterite $\mathrm{SnO}_{2}$ and their pressure and temperature-dependence. J Geophys Res 80:2595-2599

123. Rohrbach A, Hafner J, Kresse G (2004) Ab initio study of the (0001) surfaces of hematite and chromia: influence of strong electronic correlations. Phys Rev B 70:125426 


\title{
Testing one-parameter hybrid exchange functionals in confined atomic systems
}

\author{
Francisco-Adrián Duarte-Alcaráz ${ }^{1} \oplus$, Michael-Adán Martínez-Sánchez $^{2} \oplus$, \\ Marcos Rivera-Almazo ${ }^{2}$ (1) , Rubicelia Vargas $^{2}$ (i), \\ Rodrigo-Arturo Rosas-Burgos ${ }^{3}$ (i) and Jorge Garza ${ }^{2}$ (i) \\ ${ }^{1}$ Departamento de Investigación en Física. División de Ciencias Exactas y Naturales. Universidad de \\ Sonora. Blvd. Luis Encinas y Rosales S/N, Col. Centro. Hermosillo. C.P. 83000. Sonora. México \\ ${ }^{2}$ Departamento de Química, División de Ciencias Básicas e Ingeniería. Universidad Autónoma \\ Metropolitana-Iztapalapa. San Rafael Atlixco 186, Col. Vicentina. Iztapalapa. C. P. 09340. Ciudad de \\ México. México \\ ${ }^{3}$ Departamento de Física, Universidad de Sonora. Blvd. Luis Encinas y Rosales S/N, Col. Centro. \\ Hermosillo. C.P. 83000 Sonora, México \\ E-mail: jgo@xanum.uam.mx
}

Received 14 January 2019, revised 1 May 2019

Accepted for publication 21 May 2019

Published 18 June 2019

\begin{abstract}
In this work the Perdew-Burke-Ernzerhof exchange functional coupled with the exact-exchange is applied on closed-shell atoms confined by impenetrable and penetrable walls. When the Hartree-Fock method is used as the reference, one-parameter hybrid exchange functionals qualitatively give a good description of atoms enclosed by a sphere surrounded by an infinite potential. For atoms confined by a finite potential, however, the same hybrid exchange functionals predict results appreciably different to the reference for small confinement radii. The main reason of this result is the Laplacian of the electron density involved in the exchange potential and, consequently, the effective potential diverges at the nucleus, which cannot be remedied by the inclusion of the exact exchange. Localization and delocalization exhibited by the electron density are used as arguments to explain the differences found between various exchange functionals tested in this article. We show that generalized-gradient functionals are unable to give a good description of the corresponding system when the electron density is squeezed by finite potentials over small regions and how one-parameter hybrid exchange functionals alleviate some of the encountered problems. Although the model of the confined atom is extremely simple, it can reproduce some features predicted by sophisticated methods of electronic structure designed for crystal systems, therefore, this model can be useful to test exchange functionals defined within the Kohn-Sham density functional-theory.
\end{abstract}

Supplementary material for this article is available online

Keywords: confined atoms, density functional theory, hybrid exchange functionals

(Some figures may appear in colour only in the online journal)

\section{Introduction}

Electrons in free atoms do not present spatial limitations, and for atoms with spatial restrictions the model of the confined atom has been an useful tool to study effects on the electronic structure when their electrons are restricted to explore only some regions of the space [1-5]. Important applications are known from dense plasma physics and high-energy density physics [6] (dense astrophysics, inertial fusion science, dense laser produced plasmas) and dense plasma spectroscopy [7]; systems under extreme conditions [8] where numerous methods have successfully been applied to take into account the impact of the dense plasma on the atomic structure: the Ecker-Kröll model [9], the Stewart-Pyatt model [10, 11], the 
finite temperature ion sphere models [12, 13], the atomicsolid-plasma model [14] and the VAAQP-model [15]. For a long time, restrictions over atoms have been modeled by different potentials. For example, there is one potential where a spherical surface envelops the nucleus and the electrons within an impenetrable wall to simulate atoms under extreme pressures. The description of electrons confined by this potential has been tackled by wave-function methods [4, 16-22] or by the density functional theory [23-28], and for both cases this potential involves complications to obtain the wave-function or its corresponding electron density, since these quantities are zero on arbitrary radii and consequently many codes widely used by chemists or physicists are inoperative. The design of new codes is, therefore, required to attack this problem.

As an alternative to walls with infinite potential, people have designed potentials with penetrable walls [24, 29-31]. In general, for an atom with nuclear charge $Z$ we can represent the external potential as

$$
\begin{aligned}
v(r) & =-\frac{Z}{r} \quad r<R_{c}, \\
& =v_{c}(r) \quad r \geqslant R_{c} .
\end{aligned}
$$

Along this article all quantities are expressed in atomic units (au). We can mention three cases for penetrable potentials: $v_{c}(r)=U_{0}[29,30], v_{c}(r)=-Z /(\epsilon r)[32,33]$ and $v_{c}(r)=$ $\omega_{0}^{2} r^{2},[34,35]$ where $U_{0}, \epsilon$ and $\omega_{0}$ are fixed for particular problems that have been used to mimic a specific environment around an atom. These potentials involve complications in the quest for the corresponding wave-function or electron density because the asymptotic behavior required by the wave-function is not necessarily the same than the one of free atoms. Fortunately, some of these potentials allow an analytical solution for one-electron systems [29, 33], and from this point, some basis set functions can be proposed to study many-electron atoms [33, 36]. Martínez-Sánchez et al have reported recently significant differences between results obtained by density functional theory, in its Kohn-Sham (KS) version, and those obtained from the Hartree-Fock (HF) method for atoms confined by a constant potential, $U_{0}$ [28]. In that report, Martínez-Sanchez et al solved the KS equations with the same Roothaan's approach used to solve HF equations. A relevant conclusion by Martínez-Sánchez et al [28] is related to the exchange energy (XE) provided by HF and KS through the local density approximation (LDA) [37] and the generalized gradient approximation (GGA) within the proposal by Becke (B88) [38]. To illustrate this point in this section, we will use results already published elsewhere, in particular, the exchange energy obtained for atoms confined by impenetrable and penetrable walls is an important property to analyze.

Figure 1(a) presents results reported in the table 1 of [25] and the table 3 of [28] for a He atom. In this figure the exchange energy is presented for HF in two situations: (1) dotted line for penetrable walls, (2) squares for impenetrable walls. For impenetrable walls this confinement decreases the exchange energy, as we can appreciate it from the squares in this plot. The situation is different for penetrable walls since

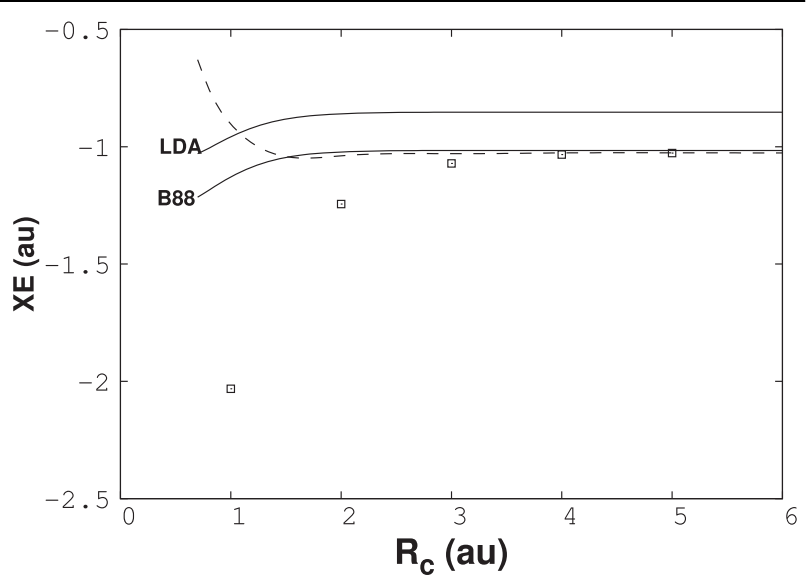

(a)

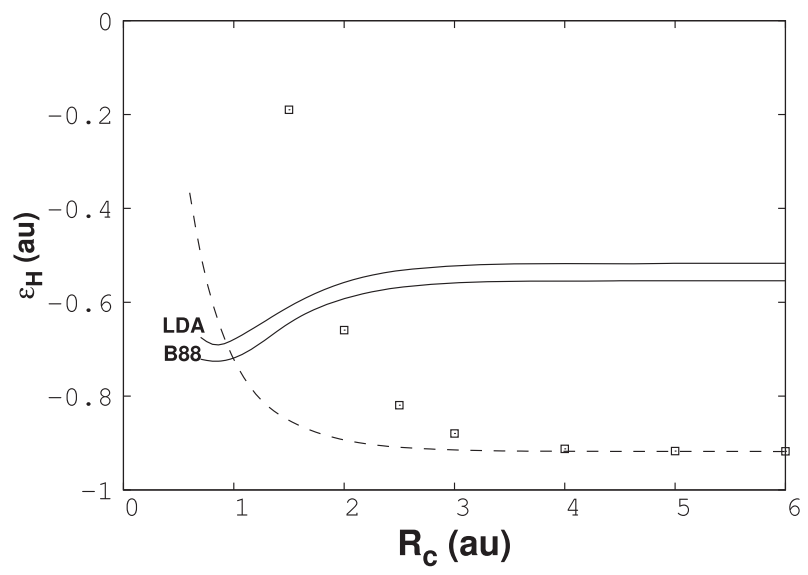

(b)

Figure 1. Helium atom confined by penetrable (lines) and impenetrable (squares) walls. Dotted lines and squares represent the HF results and solid lines refer to KS results. (a) Exchange energy, (b) highest occupied atomic orbital. The HF results for impenetrable walls are from [25]. HF, LDA, and B88 results, from [28].

this confinement increases the exchange energy when the corresponding radius exhibits small values $[36,39]$, which is evidenced by the dashed line. LDA and B88 exchange functionals predict different results to those obtained from HF. In figure 1(a), these exchange functionals were applied to the same atom confined by penetrable walls $\left(U_{0}=0.0 \mathrm{au}\right)$. From figure 1(a), the exchange energy obtained by these functionals behaves similarly to the impenetrable confinement, since both decrease the exchange energy when a constant potential confines the atom. From this discussion, it is reasonable to think that orbital energies must be sensitive to the changes observed by the exchange contribution through its corresponding potential. The highest occupied atomic orbital, $\epsilon_{H}$, obtained from the methods mentioned in this section, is presented in figure 1(b). From this figure, we may observe that the confinement increases $\epsilon_{H}$ when HF is used either for penetrable or for impenetrable walls. Also, it is clear that LDA or B88 exchange functionals do not recover such a 
behavior. Unfortunately, Martínez-Sánchez et al did not explain the differences found between HF and KS [28].

From the above it is evident that the model of the confined atom can be used to test the performance of exchangecorrelation functionals. Typically, the design of exchangecorrelation functionals is followed by its test over several atomic and molecular systems, [40] or even for periodic systems [41]. In this article, the main target is the analysis of exchange functionals that contain a fraction of the exact exchange (EE) in the description of atoms confined by penetrable or impenetrable walls. For instance, an exchangeonly study will be reported here using HF results as the reference. The efforts of several colleagues that design exchange-correlation functionals based on physical restrictions indicate that the Perdew-Burke-Ernzerhof (PBE) exchange functional is a good starting point for the incorporation of the exchange contribution [42]. There are also reports that show that hybrid exchange functionals correct several deficiencies presented by GGA proposals, and in particular, PBE0 has been used as an alternative to obtain the electronic structure of atoms, molecules, and solids [43]. Thus, in this article, we will treat one-parameter hybrid exchange functionals obtained from $\mathrm{PBE}$ and the EE through the expression

$$
E_{x}^{\mathrm{hyb}}=E_{x}^{\mathrm{PBE}}+\alpha\left(\mathrm{EE}-E_{x}^{\mathrm{PBE}}\right),
$$

where $\alpha$ is a parameter to tune the contribution of the EE. Sometimes $\alpha$ is related to the adiabatic connection or to $1 / n$, where $n$ is an integer. The PBE0 functional is obtained when $n=4$; for $n=2$ we obtain PBE0-1/2, PBE013 for $n=3$, PBE- $1 / 5$ for $n=5$ and so on [44, 45]. In this article, $\alpha$ will be used as one parameter between 0 and 1 .

\section{Methodology}

The KS total energy (TE) [46] is obtained from

$$
E_{\mathrm{KS}}=T_{s}+J+E_{x}^{\mathrm{hyb}}+\int d \mathbf{r} v(\mathbf{r}) \rho(\mathbf{r}),
$$

where $T_{s}$ represents the kinetic energy associated to a noninteracting particles system

$$
T_{s}=\sum_{i=1}^{N}\left\langle\chi_{i}\left|-\frac{1}{2} \nabla^{2}\right| \chi_{i}\right\rangle,
$$

and

$$
J=\frac{1}{2} \iint d \mathbf{r} d \mathbf{r}^{\prime} \frac{\rho(\mathbf{r}) \rho\left(\mathbf{r}^{\prime}\right)}{\left|\mathbf{r}-\mathbf{r}^{\prime}\right|}
$$

represents the electrostatic energy provided by the electron density. The PBE exchange contribution in equation (2) has the form

$$
E_{x}^{\mathrm{PBE}}=\int d \mathbf{r} \rho \epsilon_{x}^{\mathrm{unif}}(\rho) F_{x}\left(r_{s}, \xi, s\right),
$$

with

$$
\epsilon_{x}^{\mathrm{unif}}(\rho)=-\frac{3 k_{f}}{4 \pi}
$$

$$
\begin{gathered}
k_{f}=\left(3 \pi^{2} \rho\right)^{1 / 3}, \\
F_{x}(s)=1+\kappa-\frac{\kappa}{1+\frac{\mu_{x}}{\kappa} s^{2}}, \\
s=\frac{|\nabla \rho|}{2 k_{f} \rho},
\end{gathered}
$$

$\mu_{x}=0.21951$ and $\kappa=0.804$. The EE has the expression

$$
\mathrm{EE}=-\frac{1}{2} \sum_{i=1}^{N} \sum_{j=1}^{N} \iint \mathrm{d} \mathbf{x} \mathrm{d} \mathbf{x} \mathbf{x}^{\prime} \frac{\chi_{i}^{*}(\mathbf{x}) \chi_{j}^{*}\left(\mathbf{x}^{\prime}\right) \chi_{j}(\mathbf{x}) \chi_{i}\left(\mathbf{x}^{\prime}\right)}{\left|\mathbf{r}^{\prime}-\mathbf{r}\right|},
$$

with spin-orbitals, $\left\{\chi_{i}(\mathbf{x})\right\}$, written as $\chi(\mathbf{x})=\psi(\mathbf{r}) \sigma(\omega)$ and $\sigma=\alpha, \beta$. Finally, the external potential, $v$, is represented by equation (1) with $v_{c}(r)=U_{0}$.

The spin-orbitals, $\left\{\chi_{i}(\mathbf{x})\right\}$, that minimize equation (3) must satisfy

$$
\begin{gathered}
\left(-\frac{1}{2} \nabla^{2}+v(\mathbf{r})+\int d \mathbf{r}^{\prime} \frac{\rho\left(\mathbf{r}^{\prime}\right)}{\left|\mathbf{r}-\mathbf{r}^{\prime}\right|}+(1-\alpha) \frac{\delta E_{x}^{\mathrm{PBE}}}{\delta \rho(\mathbf{r})}\right. \\
\left.+\alpha v^{\mathrm{EE}}\right) \chi_{i}(\mathbf{x})=\epsilon_{i} \chi_{i}(\mathbf{x})
\end{gathered}
$$

with

$$
v^{\mathrm{EE}} \chi_{i}(\mathbf{x})=-\sum_{j=1}^{N} \int \mathrm{d} \mathbf{x}^{\prime} \frac{\chi_{j}^{*}\left(\mathbf{x}^{\prime}\right) \chi_{i}\left(\mathbf{x}^{\prime}\right)}{\left|\mathbf{r}-\mathbf{r}^{\prime}\right|} \chi_{j}(\mathbf{x}),
$$

and the electron density is written as

$$
\rho(\mathbf{r})=\sum_{i=1}^{N} \psi_{i}^{*}(\mathbf{r}) \psi_{i}(\mathbf{r}) .
$$

In this article, the Roothaan's approach [47] is used to solve the KS equations, therefore

$$
\psi_{i}(\mathbf{r})=\sum_{\mu=1}^{K} c_{\mu}^{i} \phi_{\mu}(\mathbf{r}),
$$

with $\left\{\phi_{i}(\mathbf{r})\right\}$ as the basis set, and the coefficients $\left\{c_{\mu}^{i}\right\}$ of this linear combination are obtained from the solution of the generalized eigenvalue problem

$$
\mathbf{F C}=\mathrm{SC} \epsilon .
$$

By the form of the external potential (equation (1)), the basis set is written in terms of a radial part, $f_{\mu}(r)$, times a spherical harmonic function

$$
\phi_{\mu}(\mathbf{r})=Y_{l_{\mu}, m_{\mu}}(\Omega) f_{\mu}(r),
$$

with $f_{\mu}(r)$ defined in two parts [36]

$$
\begin{array}{rlrl}
f_{\mu}(r) & =f^{-}(r)=N_{\mu}^{-} r^{n_{\mu}-1} e^{-\zeta_{\mu} r} & r<R_{c}, \\
& =f^{+}(r)=N_{\mu}^{+} r^{-l_{\mu}-1} e^{-\alpha_{\mu} r} & r \geqslant R_{c} .
\end{array}
$$

The continuity at $r=R_{c}$ of the wave-function is imposed by

$$
f^{-}\left(r=R_{c}\right)=f^{+}\left(r=R_{c}\right),
$$

and the logarithmic derivative

$$
\left.\frac{1}{f^{-}} \frac{d f^{-}}{d r}\right|_{r=R_{c}}=\left.\frac{1}{f^{+}} \frac{d f^{+}}{d r}\right|_{r=R_{c}} .
$$


Table 1. Total energy (TE) and exchange energy (XE) for free atoms obtained by MEXICA-C and NWChem codes. The aug-cc-pVQZ basis set was used with NWChem. All quantities are in atomic units.

\begin{tabular}{|c|c|c|c|c|}
\hline \multirow[b]{2}{*}{ Atom } & \multicolumn{2}{|c|}{ MEXICA-C } & \multicolumn{2}{|c|}{ NWChem } \\
\hline & TE & $\mathrm{XE}$ & TE & $\mathrm{XE}$ \\
\hline \multicolumn{5}{|c|}{ PBE } \\
\hline $\mathrm{He}$ & -2.85204 & -1.00164 & -2.85188 & -1.00160 \\
\hline $\mathrm{Be}$ & -14.54501 & -2.62746 & -14.54497 & -2.62747 \\
\hline $\mathrm{Ne}$ & -128.52009 & -12.00834 & -128.51757 & -12.00780 \\
\hline $\mathrm{Mg}$ & -199.54678 & -15.88070 & -199.54498 & -15.88504 \\
\hline $\mathrm{Ar}$ & -526.64267 & -29.95586 & -526.64030 & -29.95663 \\
\hline \multicolumn{5}{|c|}{ PBE0 } \\
\hline $\mathrm{He}$ & -2.85394 & -1.00687 & -2.85379 & -1.00683 \\
\hline $\mathrm{Be}$ & -14.55141 & -2.63624 & -14.55137 & -2.63626 \\
\hline $\mathrm{Ne}$ & -128.52393 & -12.02887 & -128.52145 & -12.02844 \\
\hline $\mathrm{Mg}$ & -199.56148 & -15.90502 & -199.56030 & -15.90889 \\
\hline $\mathrm{Ar}$ & -526.68363 & -30.00837 & -526.68202 & -30.00969 \\
\hline \multicolumn{5}{|c|}{$\mathrm{HF}$} \\
\hline $\mathrm{He}$ & -2.86168 & -1.02577 & -2.86154 & -1.02571 \\
\hline $\mathrm{Be}$ & -14.57302 & -2.66691 & -14.57298 & -2.66690 \\
\hline $\mathrm{Ne}$ & -128.54710 & -12.10835 & -128.54469 & -12.10805 \\
\hline $\mathrm{Mg}$ & -199.61464 & -15.99429 & -199.61424 & -15.99428 \\
\hline $\mathrm{Ar}$ & -526.81751 & -30.18494 & -526.8169 & -30.18469 \\
\hline
\end{tabular}

The proposal to the radial part of equation (18) was inspired by the analytical solution of the Schrödinger equation associated to the hydrogen atom confined by a constant potential [29]. Such a basis set has been implemented in the MEXICA$\mathrm{C}$ code to solve $\mathrm{HF}$ and $\mathrm{KS}$ equations [19, 28, 36, 48]. Besides, in this article we obtain the effect of the EE in atoms confined by hard walls using the basis set

$$
f_{\mu}=C_{\mu} e^{-\xi_{\mu} r}\left(1-\frac{r}{R_{c}}\right) .
$$

These functions cancel the electron density on the walls, and they are already implemented in the MEXICA-C code $[4,19,36]$. The exponents, $\left\{\zeta_{i}\right\}$, involved in the basis set must be optimized for each confinement radii. Details about the grid used to compute Fock matrix elements can be found in [28]. The basis set used in this article was obtained from [36] where the HF method was used to optimize the basis set for five closed-shell atoms ( $\mathrm{He}, \mathrm{Be}, \mathrm{Ne}, \mathrm{Mg}$, and $\mathrm{Ar}$ ) and two values for $U_{0}: 0.0$ and $0.5 \mathrm{au}$. In the electronic supplementary information (ESI) is available online at stacks.iop.org/JPB/ $52 / 135002 /$ mmedia we are reporting some exponents for basis sets of a few confinement radii.

We must mention one important approximation made by Rodriguez-Bautista et al [36] in the implementation of the Roothaan's approach to solve HF equations, which is based on the proposal made by Gorecki and Byers-Brown [30]: the twoelectrons integrals are zero for $r \geqslant R_{c}$. This means that the Coulomb and the exchange energy contributions are zero in this region. In other words, the electron-electron interaction is neglected within the constant potential. Thus, if we want to compare $\mathrm{HF}$ and $\mathrm{KS}$ results then the exchange-correlation potential must be zero for $r \geqslant R_{c}$, and the corresponding exchange-correlation energy must be also zero within this region. Martínez-Sánchez et al did not considered this approximation and consequently the exchange-correlation contribution was overestimated in [28]. For the energy related to one-parameter hybrid exchange functionals (equation (2)), we canceled two-electron integrals for $\mathrm{EE}$ and Coulomb contributions for $r \geqslant R_{c}$, and the exchange energy from PBE is also zero for this region. Thus, we did not consider the electron-electron interaction inside of the constant potential. Otherwise, we need to specify a model to take into account this interaction.

\section{Results and discussion}

Total and exchange energies for five closed-shell free atoms are reported in table 1 since we have implemented the PBE exchange functional into the MEXICA-C code and we want to compare its results with other codes. In this case, we used the basis set reported by Thakkar et al [49] for the NWChem [50], the aug-cc-pVQZ basis set [51] was used for all atoms considered here. From table 1, we can appreciate small differences between both basis sets, although MEXICA-C code delivers total energies lower than those obtained by NWChem. To compare these results we must bear in mind that, for free atoms, MEXICA-C uses Slater-type orbitals and NWChem uses Gaussian functions.

For this set of atoms, PBE slightly underestimates the exchange energy when HF is used as the reference. Such a prediction is improved when a fraction of $\mathrm{EE}$ is incorporated in one-parameter hybrid exchange functionals, which is evidenced 


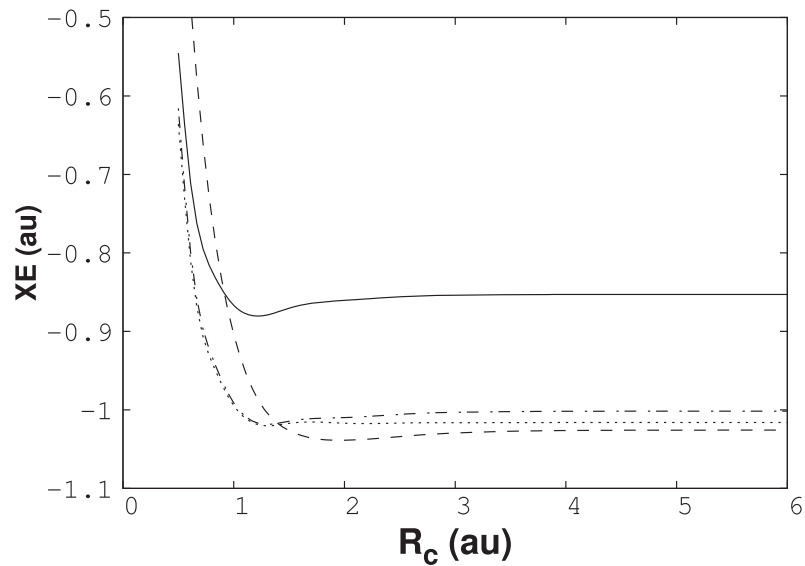

Figure 2. Exchange energy for helium atom confined by a constant potential $U_{0}=0.0$ au. LDA: solid line. B88: dotted line. PBE: dotted-dashed line. HF: dashed line.

by the PBE0 functional. From this point, it is interesting to know if such differences are maintained when electrons in these atoms are confined by infinite or finite potentials.

\subsection{Atoms confined by penetrable walls}

The helium atom is the first system to analyze, and after these results, general conclusions will be mentioned from all the atoms studied here. In the first instance, PBE results will be compared with those obtained by LDA and B88 functionals.

The exchange energy obtained by LDA, B88, PBE, and $\mathrm{HF}$ is depicted in figure 2 for the helium atom confined by a constant potential $\left(U_{0}=0.00 \mathrm{au}\right)$ for $R c \geqslant 0.6 \mathrm{au}$. The comparison between figures 1 (a) and 2 is the first result to discuss. In figure 2 we observe that exchange functionals follow the same trend than that presented by HF. This result is contrary to that presented in figure 1(a) by LDA and B88 functionals. Thus, the results reported by Martínez-Sánchez et al overestimated the exchange energy since the integral to evaluate this quantity was not restricted to $r \leqslant R_{c}$ [28]. From figure 2 three exchange correlation functionals give the same behavior of the exchange energy obtained by HF, although we can find one important difference: there are regions where these functionals predict lower exchange energies than those predicted by HF, even for LDA. Let us explain this result.

For the helium atom in its ground state, equation (11) is reduced to

$$
\mathrm{EE}=-\frac{1}{4} \iint d \mathbf{r} d \mathbf{r}^{\prime} \frac{\rho(\mathbf{r}) \rho\left(\mathbf{r}^{\prime}\right)}{\left|\mathbf{r}-\mathbf{r}^{\prime}\right|}
$$

or

$$
\mathrm{EE}=\int d \mathbf{r} \rho(\mathbf{r})\left(-\frac{1}{4} \int d \mathbf{r}^{\prime} \frac{\rho\left(\mathbf{r}^{\prime}\right)}{\left|\mathbf{r}-\mathbf{r}^{\prime}\right|}\right),
$$

which is zero for $r \geqslant R_{c}$. Thus, we will analyze the behavior of the exchange energy presented in figure 2 contrasting equation (23) with equation (6). For convenience, from equation (6) we define

$$
e_{x}^{\mathrm{PBE}}=\epsilon_{x}^{\mathrm{unif}}(\rho) F_{x}\left(r_{s}, \xi, s\right),
$$

and from equation (23)

$$
e_{x}^{\mathrm{HF}}=-\frac{1}{4} \int d \mathbf{r}^{\prime} \frac{\rho\left(\mathbf{r}^{\prime}\right)}{\left|\mathbf{r}-\mathbf{r}^{\prime}\right|}
$$

In fact, from equation (6) we can also define

$$
e_{x}^{\mathrm{LDA}}=\epsilon_{x}^{\mathrm{unif}}(\rho) .
$$

These definitions show that the possible differences observed on the exchange energy among LDA, PBE and HF come from differences associated with $e_{x}^{\mathrm{LDA}}, e_{x}^{\mathrm{PBE}}$ and $e_{x}^{\mathrm{HF}}$.

The comparison between $e_{x}^{\mathrm{LDA}}, e_{x}^{\mathrm{PBE}}$ and $e_{x}^{\mathrm{HF}}$ is presented in figure 3. From this figure, we observe that there is a gray region where $e_{x}^{\mathrm{HF}}>e_{x}^{\mathrm{LDA}}, e_{x}^{\mathrm{PBE}}$. For small confinement radii, this behavior induces $E_{x}^{\mathrm{HF}}>E_{x}^{\mathrm{LDA}}, E_{x}^{\mathrm{PBE}}\left(\left|E_{x}^{\mathrm{HF}}\right|<\left|E_{x}^{\mathrm{LDA}}\right|\right.$, $\left.\left|E_{x}^{\mathrm{PBE}}\right|\right)$. This result suggests that the TE predicted by exchange functionals will be lower than that predicted by HF. The TE exhibits, for our example, the following values for $R_{c}=0.6 \mathrm{au}: E_{\mathrm{KS}}(\mathrm{LDA})=-1.42857 \mathrm{au}, E_{\mathrm{KS}}(\mathrm{PBE})=$ $-1.49268 \mathrm{au}, E_{\mathrm{KS}}(\mathrm{HF})=-1.19418 \mathrm{au}$. Thus, the overestimation of $e_{x}$ by the exchange functional approximations for $r \approx 0$ produces total energies that are lower than the reference one (HF) for small confinement radii. For large confinement radii, the EE dominates on the asymptotic region (as we can observe in figure 3) and, consequently, the exchange energy follows the behavior $E_{x}^{\mathrm{HF}}<E_{x}^{\mathrm{LDA}}, E_{x}^{\mathrm{PBE}}$ $\left(\left|E_{x}^{\mathrm{HF}}\right|>\left|E_{x}^{\mathrm{LDA}}\right|,\left|E_{x}^{\mathrm{PBE}}\right|\right)$. For small confinement radii PBE overestimates the exchange energy in $70.0 \%$ for $R_{c}=0.6 \mathrm{au}$, and this functional underestimates this property only in $2.4 \%$ for $R_{c}=10.0 \mathrm{au}$, as this is reported in table 2 . In the same table we observe that these differences are mapped on the TE, since for $R_{c}=0.6$ au there is an overestimation of $25.0 \%$, and $0.3 \%$ for $R_{c}=10 \mathrm{au}$. Thus, for small confinement radii, PBE and LDA fail to describe the exchange energy correctly; in fact, B88 exhibits the same deficiency, when we compare these exchange functionals with HF. In conclusion: LDA and PBE cannot reproduce EE values for small confined radii because $e_{x}^{\mathrm{LDA}}$ and $e_{x}^{\mathrm{PBE}}$ are unable to reproduce the $e_{x}^{\mathrm{HF}}$ behavior close to the nucleus.

In figure 1(b) we showed $\epsilon_{H}$ obtained by LDA, B88, and HF. Now in figure 4 we are presenting a similar plot including PBE results and evaluating all exchange functionals with the modification of our code to compute the exchange energy correctly. A comparison between figures 1(b) and 4 indicates that if we do consider the electron-electron interaction within the constant potential, as Martínez et al did it, then $\epsilon_{H}$ will be overestimated. However, in both cases, the possible ionization of $\mathrm{He}$ by the action of the confinement will be observed first for $\mathrm{HF}$, and exchange functionals will need confinement radii smaller than that observed for HF. Therefore, exchange functionals as LDA, B88, and PBE do not describe rightly orbital energies for helium confined by a constant potential.

From here it is evident that LDA and GGA do not provide correct exchange energy and $\epsilon_{H}$ for the helium atom confined by penetrable walls, although GGA predicts reasonably well the exchange energy for free atoms. Now let us 

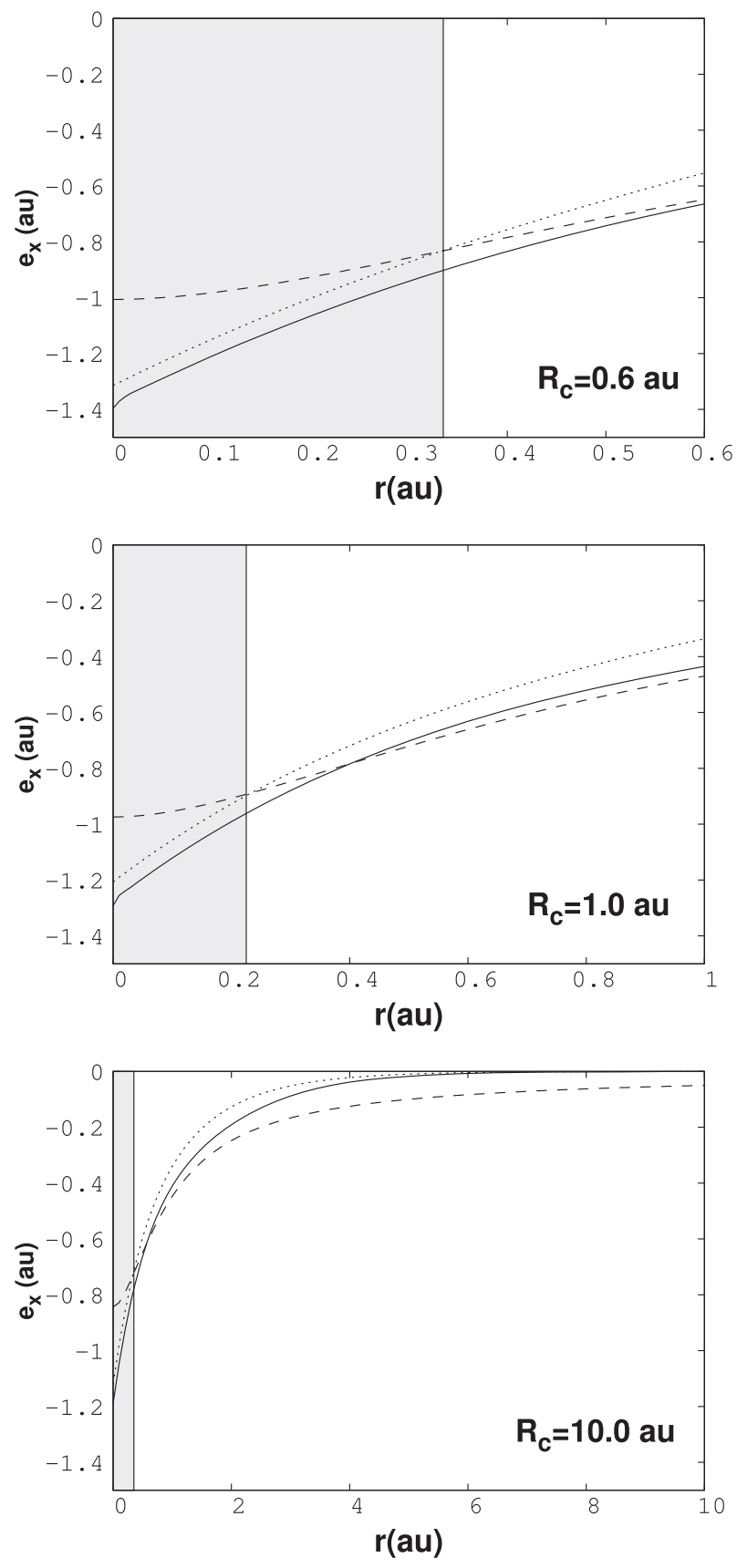

Figure 3. Local exchange energy per particle for helium atom confined by a constant potential $U_{0}=0.0 \mathrm{au}$, for three confinement radii, $R_{c}: 0.6,1.0$ and 10.0 au. Solid line for PBE, dashed line for HF and dot-dash line for LDA.

see if a portion of the EE alleviates this problem, for this reason, we report the TE, the exchange energy and $\epsilon_{H}$ in table 2 for some confinement radii and a few values of $\alpha$ using one-parameter hybrid exchange functionals defined in equation (2).

The values assigned to $\alpha$ in table 2 are important since they represent PBE, PBE0, PBE0-1/2, and HF methods. These results show the impact of the EE on TE, XE and $\epsilon_{H}$ over the confined helium atom. However, to gain a better understanding of one-parameter hybrid exchange functionals over the helium confined by a finite potential we present XE in figure 5 for several confinement radii and ten values of $\alpha$.

From figure 5 it is evident the impact of the fraction of EE on one-parameter hybrid exchange functionals. From this plot we observe that there are three regions for $R_{c}$ where hybrid functionals exhibit different behaviors; in one region these functionals underestimate the exchange energy and in another region they overestimate this property. It is worth noting that there is one small region where all hybrid functionals give almost same results than HF. In general, the incorporation of the EE corrects in the right direction the prediction of the exchange energy for all confinement radii. In this plot, $\operatorname{PBE}(\alpha=0)$ and $\mathrm{HF}(\alpha=1)$ act as upper or lower bounds, which depend on the confinement radius $R_{c}$. For this two-electron system, the one-parameter hybrid exchange functional has the form

$$
E_{x}^{\mathrm{hyb}}=\int d \mathbf{r} \rho(\mathbf{r})\left\{(1-\alpha) e_{x}^{\mathrm{PBE}}+e_{x}^{\mathrm{HF}}\right\},
$$

with $e_{x}^{\mathrm{PBE}}$ and $e_{x}^{\mathrm{HF}}$ defined in equations (24) and (25), respectively. Results presented in figure 5 show that $e_{x}^{\mathrm{PBE}}$ dominates for small confinement radii $\left(R_{c} \approx 1.3 \mathrm{au}\right)$ and consequently the prediction for the exchange energy is lower than the one obtained by the reference (HF).

We present $\epsilon_{H}$ in figure 6 as a function of $\alpha$ defined in equation (2) for several confinement radii when $U_{0}=0.0 \mathrm{au}$. From this figure we observe that for moderate confinements the orbital energy $\epsilon_{H}$ does not show appreciable differences regarding the free atom. Thus, in this region, PBE underestimates this property and it exhibits a relative error of 0.40 , which is large considering that $\mathrm{HF}$ predicts $\epsilon_{H}=-0.9180 \mathrm{au}$. From this figure, we appreciate that $E_{x}^{\text {hyb }}$ takes $\epsilon_{H}$ in the right direction. However, for small confinement radii, all one-parameter exchange functionals give similar results to PBE: all of them predict $\epsilon_{H}$ lower than that predicted by HF. We can corroborate this statement from inset of figure 6 and table 2. To give an explanation of this result we must analyze the exchange potentials considered in this article. Fortunately, a two-electron system has a closed-form for the exchange energy in terms of the electron density or the EE potential. For the closed-shell helium atom we have

$$
v^{\mathrm{EE}} \chi_{1}(\mathbf{x})=-\sum_{j=1}^{2} \int \mathrm{d} \mathbf{x}^{\prime} \frac{\chi_{j}^{*}\left(\mathbf{x}^{\prime}\right) \chi_{1}\left(\mathbf{x}^{\prime}\right)}{\left|\mathbf{r}-\mathbf{r}^{\prime}\right|} \chi_{j}(\mathbf{x}),
$$

or

$$
\begin{gathered}
v^{\mathrm{EE}} \psi(\mathbf{r})=-\int \mathrm{d} \mathbf{x}^{\prime} \frac{\psi^{*}(\mathbf{r}) \psi(\mathbf{r})}{\left|\mathbf{r}-\mathbf{r}^{\prime}\right|} \psi(\mathbf{r}), \\
=-\int \mathrm{d} \mathbf{r}^{\prime} \frac{\left|\psi\left(\mathbf{r}^{\prime}\right)\right|^{2}}{\left|\mathbf{r}-\mathbf{r}^{\prime}\right|} \psi(\mathbf{r}),
\end{gathered}
$$

which is valid for both particles. This means that for this system

$$
v^{\mathrm{EE}}=-\int \mathrm{d} \mathbf{r}^{\prime} \frac{\left|\psi\left(\mathbf{r}^{\prime}\right)\right|^{2}}{\left|\mathbf{r}-\mathbf{r}^{\prime}\right|},
$$


Table 2. Total energy (TE), exchange energy (XE) and highest occupied atomic orbital $\left(\epsilon_{H}\right)$ for helium confined by a constant potential $\left(U_{0}=0.00 \mathrm{au}\right)$ for several confinement radii $\left(R_{c}\right)$ and four values of $\alpha$ using one-parameter hybrid exchange functionals defined in equation (2); $\alpha=0.00$ (PBE), $\alpha=0.25$ (PBE0), $\alpha=0.50$ (PBE0-1/2), and $\alpha=1.00$ (HF). All quantities are reported in atomic units.

\begin{tabular}{lcccccc}
\hline$R_{c}$ & $\mathrm{TE}$ & $\mathrm{XE}$ & $\epsilon_{H}$ & $\mathrm{TE}$ & $\mathrm{XE}$ & $\epsilon_{H}$ \\
\hline \multicolumn{3}{c}{$\alpha=0.00$} & & & $\alpha=0.25$ \\
\hline 0.6 & -1.49268 & -0.78434 & -0.3744 & -1.41767 & -0.70330 & -0.3733 \\
1.0 & -2.49475 & -0.99119 & -0.5498 & -2.45637 & -0.96522 & -0.5880 \\
1.5 & -2.77478 & -1.01439 & -0.5677 & -2.76148 & -1.01401 & -0.6318 \\
2.0 & -2.83347 & -1.00977 & -0.5603 & -2.82997 & -1.01522 & -0.6382 \\
10.0 & -2.85204 & -1.00164 & -0.5531 & -2.85394 & -1.00687 & -0.6426 \\
\hline & & $\alpha=0.50$ & & & $\alpha=1.00$ & \\
\hline 0.6 & -1.34290 & -0.62242 & -0.3717 & -1.19418 & -0.46111 & -0.3665 \\
1.0 & -2.41901 & -0.94191 & -0.6292 & -2.34751 & -0.90363 & -0.7219 \\
1.5 & -2.74959 & -1.01604 & -0.7007 & -2.72976 & -1.02633 & -0.8517 \\
2.0 & -2.82752 & -1.02202 & -0.7200 & -2.82537 & -1.03861 & -0.8934 \\
10.0 & -2.85620 & -1.01267 & -0.7333 & -2.86168 & -1.02577 & -0.9180 \\
\hline
\end{tabular}

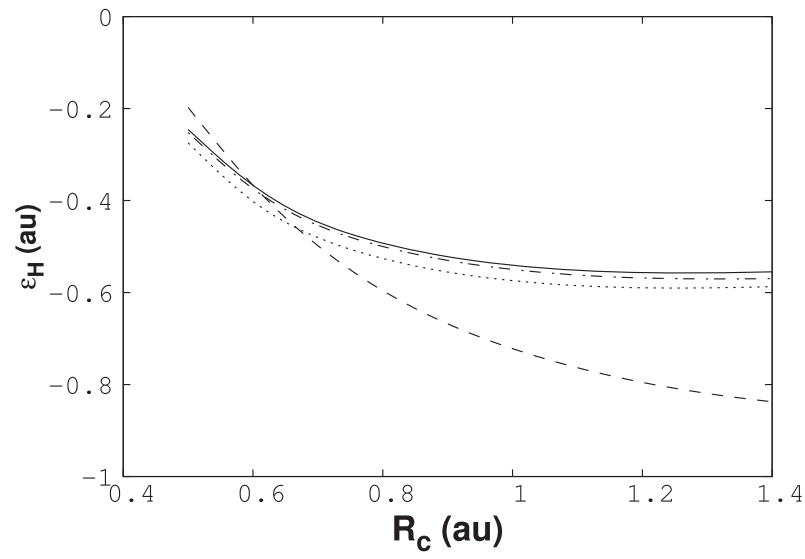

Figure 4. Highest occupied atomic orbital energy, $\epsilon_{H}$, for helium atom confined by a constant potential $U_{0}=0.0 \mathrm{au}$. LDA: solid line. Becke: dotted line. PBE: dotted-dashed line. HF: dashed line.

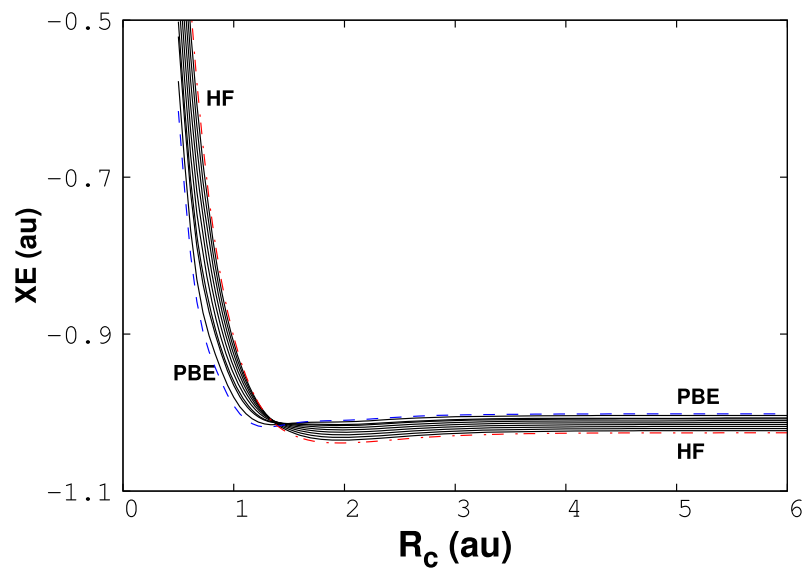

Figure 5. Exchange energy for helium atom confined by a constant potential $U_{0}=0.0$ au obtained by one-parameter exchange hybrid functionals with $\alpha=0.0,0.1, \ldots, 1.0$. PBE corresponds to $\alpha=0$ and HF to $\alpha=1$.

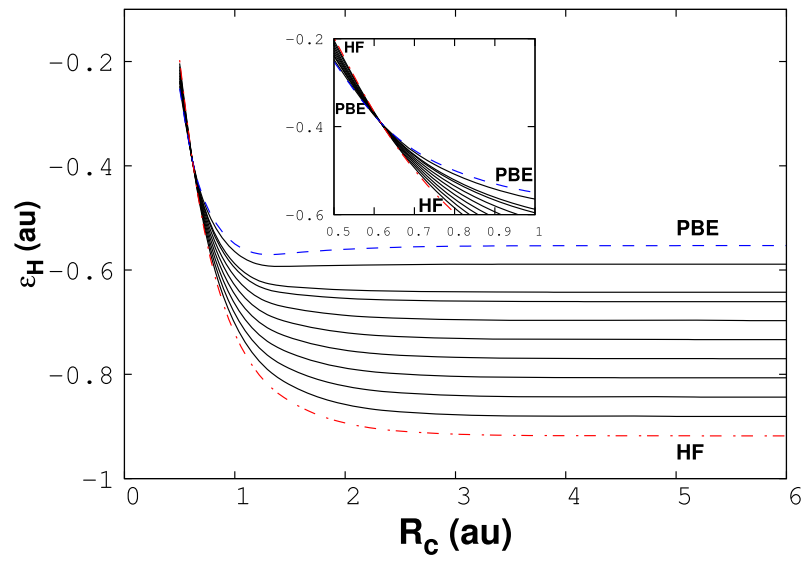

Figure 6. Highest occupied atomic orbital, $\epsilon_{H}$, for helium atom confined by a constant potential $U_{0}=0.0$ au obtained by hybrid functional with $\alpha=0.0,0.1, \ldots, 1.0$.

and consequently the one-parameter hybrid exchange potential is

$$
v_{x}^{\mathrm{Hyb}, \alpha}(\mathbf{r})=(1-\alpha) \frac{\delta E_{x}^{\mathrm{PBE}}}{\delta \rho(\mathbf{r})}-\alpha \int \mathrm{d} \mathbf{r}^{\prime} \frac{\left|\psi\left(\mathbf{r}^{\prime}\right)\right|^{2}}{\left|\mathbf{r}-\mathbf{r}^{\prime}\right|},
$$

or

$$
v_{x}^{\mathrm{Hyb}, \alpha}(\mathbf{r})=(1-\alpha) \frac{\delta E_{x}^{\mathrm{PBE}}}{\delta \rho(\mathbf{r})}-\frac{1}{2} \alpha \int \mathrm{d} \mathbf{r}^{\prime} \frac{\rho\left(\mathbf{r}^{\prime}\right)}{\left|\mathbf{r}-\mathbf{r}^{\prime}\right|} .
$$

Thus, the EE potential for two-electron systems has the form

$$
v_{x}^{\mathrm{Hyb}, \alpha=1}(\mathbf{r})=-\frac{1}{2} \int \mathrm{d} \mathbf{r}^{\prime} \frac{\rho\left(\mathbf{r}^{\prime}\right)}{\left|\mathbf{r}-\mathbf{r}^{\prime}\right|} .
$$

The exchange potential, $v_{x}^{\mathrm{Hyb}}$, for some values of $\alpha$ $(\alpha=0,0.25$ and 1$)$ is presented in figure 7. From here, we observe that $\mathrm{PBE}$ gives an exchange potential with large negative values close to the nucleus. Such a behavior is 

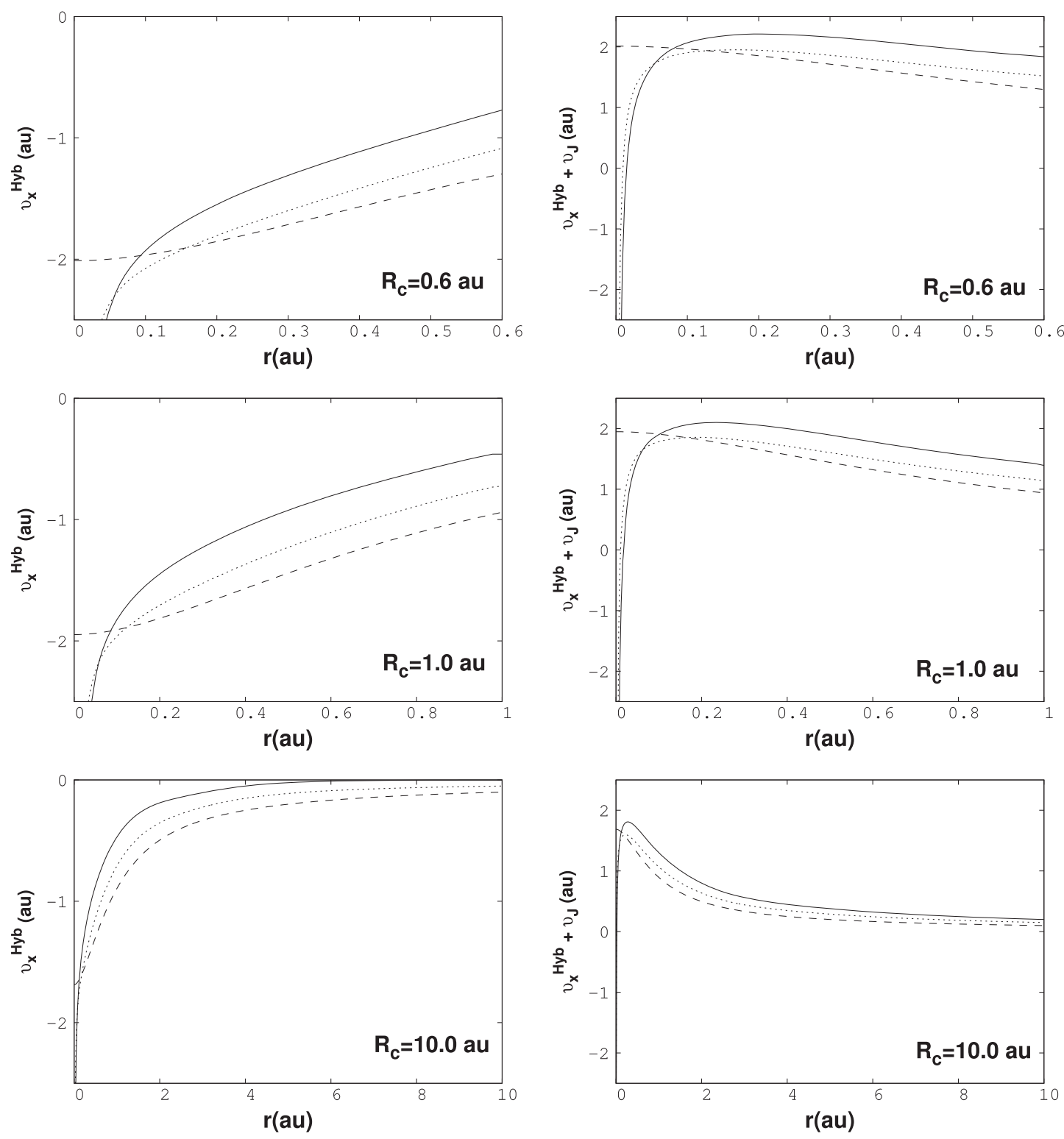

Figure 7. Exchange potential, $v_{x}^{\text {Hyb }}$, (left side) and exchange potential plus Coulomb potential, $v_{x}^{\text {Hyb }}+v_{J}$ (right side) for a helium atom confined by a constant potential $\left(U_{0}=0.0 \mathrm{au}\right)$ for three confinement radii; $R_{c}=0.6,1.0$ and $10.0 \mathrm{au}$. PBE: solid line. PBE0: dotted-dashed line. HF: dashed line.

presented because the Laplacian appears in the expression of the exchange potential and this quantity diverges at the nucleus. Evidently, this deficiency cannot be remedied by the inclusion of the EE. Thus, exchange potentials containing contributions of PBE have an incorrect behavior close to the nucleus. Therefore, for small confinement radii this spurious contribution dominates and, consequently, orbital energies are lower than that predicted by HF. For moderate or large confinement radii, the asymptotic behavior dominates and, for that reason, the HF potential gives an orbital energy lower than those delivered by one-parameter hybrid exchange potentials. Fortunately, one-parameter hybrid exchange potentials predict eigenvalues in the right way, as we can verify from table 2 . In figure 7 we have included the exchange potential plus the Coulomb potential generated by the electron density, $v_{x}^{\text {Hyb }}+v_{J}$; both contributions are important to build the effective KS potential. From these two contributions the role of the divergence involved with the PBE potential is evident for small confinement radii. This divergence is less critical for large confinement radii, where the asymptotic behavior dominates and, consequently HF orbital energies are lower than those obtained by PBE or any one-parameter hybrid exchange potential. Therefore, in case of very small confinements we expect an overestimation of eigenvalues obtained by exchange potentials containing Laplacian of the electron density. In fact, at $R_{c}=0.5$ au we obtain that $\epsilon_{H}(\alpha=0)=-0.25210 \mathrm{au}, \quad \epsilon_{H}(\alpha=0.25)=-0.23992 \mathrm{au}$, and $\epsilon_{H}(\alpha=1.0)=-0.19738 \mathrm{au}$, corroborating our argument. 


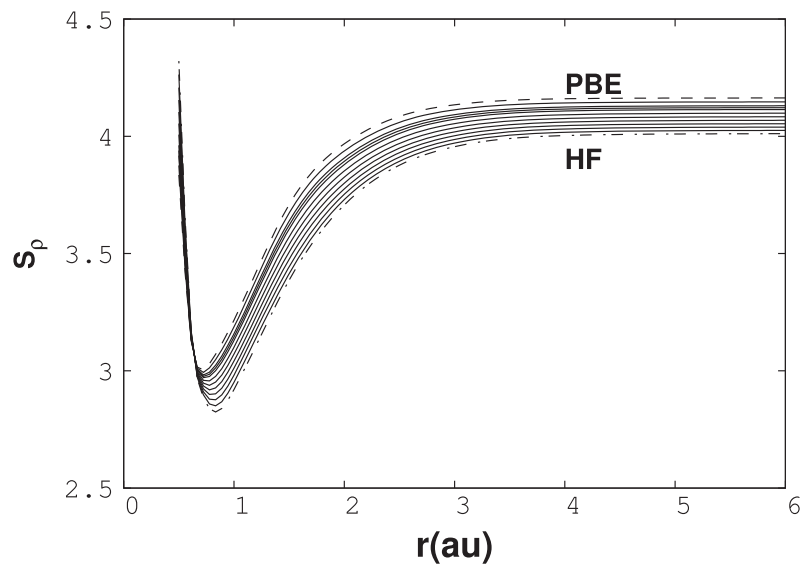

Figure 8. Shannon entropy in configuration space for helium atom confined by a finite potential with $U_{0}=0.0$ au obtained by oneparameter hybrid exchange functionals with $\alpha=0.0,0.1, \ldots, 1.0$. PBE: dashed line. HF: dotted-dashed line.

Rodriguez-Bautista et al showed that the Shannon entropy in the configuration space

$$
S_{\rho}=\int d \mathbf{r} \rho(\mathbf{r}) \ln \rho(\mathbf{r}),
$$

indicates localization or non-localization of the HF electron density for confined atoms when they compare this property with the corresponding free atom [39]. For confinements imposed by penetrable walls, these authors found that $S_{\rho}$, as a function of $R_{c}$, shows a minimum for some atoms. Besides, for small confinement radii, less than the position of this minimum $S_{\rho}$ is higher than that presented by free atoms. Let us see if this effect is present when the electron density is obtained from KS one-parameter exchange functionals; for this purpose, in figure $8 S_{\rho}$ is depicted for one-parameter hybrid exchange functionals with $\alpha=0.0,0.1,0.2, \ldots, 1.0$. From this figure we conclude that the localization or delocalization of the electron density through Shannon entropy is detected by the one-parameter exchange functionals. However, in the same figure we observe that the lowest value for $S_{\rho}$ is delivered by $\mathrm{HF}$ and the highest one is predicted by PBE. This means that the electron density from PBE is more spread than that from HF, which can be confirmed by comparing both quantities. For this reason figure 9 shows a comparison between HF electron density $(\alpha=1)$ and two electron densities obtained with $\alpha=0.25$ (PBE0) and $\alpha=0.00$ (PBE) for the helium atom confined by a constant potential with $U_{0}=0.0$ au at $R_{c}=1.0 \mathrm{au}$. The difference, $\Delta \rho=\rho^{\alpha \neq 1}-\rho^{\alpha=1}=\rho^{\alpha \neq 1}-\rho^{\mathrm{HF}}$, shows that in the asymptotic region $\rho^{\alpha \neq 1}$ dominates over $\rho^{\mathrm{HF}}$ since $\Delta \rho>0$, and therefore this electron density is more extended than that obtained with the HF method, confirming the interpretation given by the Shannon entropy.

Some values of $S_{\rho}$, included in figure 8, are reported in table 3 . These values show that the HF density is delocalized when helium atom is confined by a constant potential ( $U_{0}=0.0 \mathrm{au}$ ) at $R_{c}=0.5 \mathrm{au}$, since in this confinement the Shannon entropy is greater than that associated with the free atom. These results suggest that for one-parameter hybrid

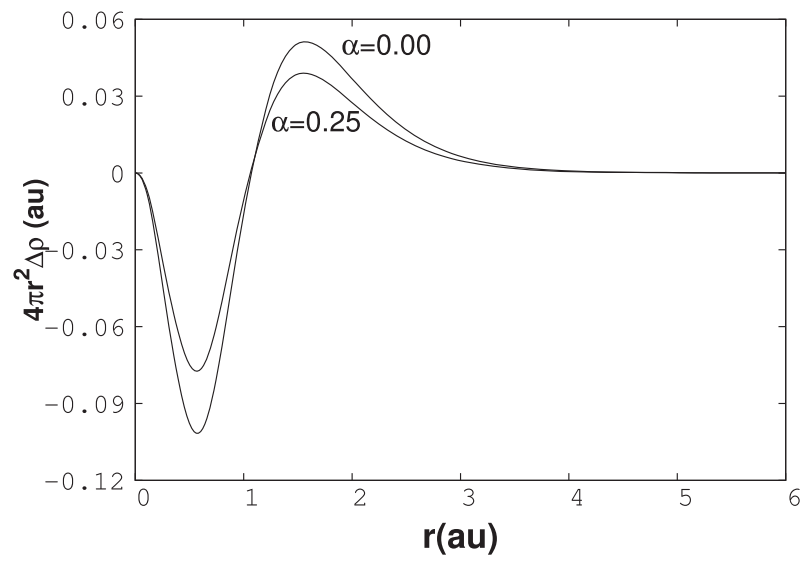

Figure 9. Electron density difference $\Delta \rho=\rho^{\alpha \neq 1}-\rho^{\alpha=1}$ for helium confined by a constant potential $\left(U_{0}=0.0 \mathrm{au}\right)$ at $R_{c}=1.0 \mathrm{au}$ for $\alpha=0.00$ and $\alpha=0.25$.

Table 3. Shannon entropy in configuration space for helium confined by a constant potential $\left(U_{0}=0.00 \mathrm{au}\right)$ for several confinement radii $\left(R_{c}\right)$ and obtained with one-parameter hybrid exchange functionals defined in equation (2).

\begin{tabular}{lcccc}
\hline $\operatorname{Rc}(\mathrm{au})$ & $\alpha=0.00$ & $\alpha=0.25$ & $\alpha=0.50$ & $\alpha=1.00$ \\
\hline 0.5 & 3.8351 & 3.9395 & 4.0544 & 4.3207 \\
1.0 & 3.2161 & 3.1448 & 3.0715 & 2.9202 \\
1.5 & 3.7088 & 3.6216 & 3.5388 & 3.3865 \\
2.0 & 3.9679 & 3.8934 & 3.8255 & 3.7072 \\
30.0 & 4.1632 & 4.1218 & 4.0828 & 4.0107 \\
\hline
\end{tabular}

exchange functionals we need very small confinement radii to delocalize the electron density. Naturally, we need to confirm such results by looking through the electron density. In figure 10 we present the electron density difference $\left.\delta \rho(r)\right|_{1.0}=\left.\rho(r)\right|_{R_{c}=1.0}-\left.\rho(r)\right|_{R_{c}=30}$. According to table 3, we expect localization of the electron density when the helium atom is confined by a constant potential $\left(U_{0}=0.0 \mathrm{au}\right)$ at $R_{c}=1.0 \mathrm{au}$ since $S_{\rho}\left(R_{c}=1.0\right)>S_{\rho}\left(R_{c}=30.0\right)$. This result is confirmed by figure 10 since in the asymptotic region the electron density obtained for $R_{c}=30$ au dominates over the same quantity for $R_{c}=1.0 \mathrm{au}$. In the same figure, we present $\left.\delta \rho(r)\right|_{0.5}=\left.\rho(r)\right|_{R_{c}=0.5}-\left.\rho(r)\right|_{R_{c}=30}$, and in this case we expect a localization of the electron density, which is confirmed by observing the bottom of figure 10 . In this plot we notice that $\left.\rho(r)\right|_{R_{c}=0.5}$ dominates over $\left.\rho(r)\right|_{R_{c}=30}$ in the asymptotic region since $\left.\delta \rho(r)\right|_{0.5}>0$.

From this discussion we conclude that one-parameter hybrid exchange functionals predict similar Shannon entropy in configuration space than HF when the helium atom is confined by a constant potential; in general, the electron density is localized with the confinement, although for very small confinements $S_{\rho}$ predicts a delocalization. However, exchange functionals predict the delocalization of electron density for confinement radii lower than those obtained by $\mathrm{HF}$. 

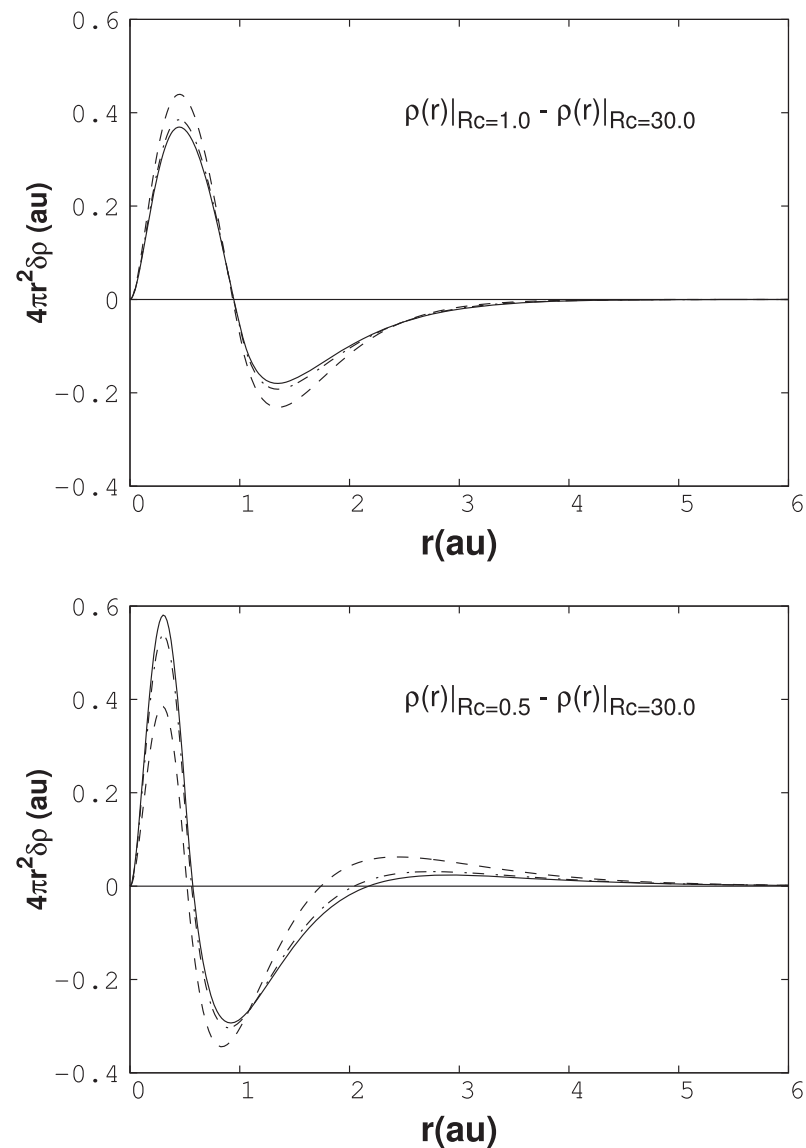

Figure 10. Electron density difference $\left.\delta \rho(r)\right|_{R_{c}}=\left.\rho(r)\right|_{R_{c}}-$ $\left.\rho(r)\right|_{R_{c}=30}$ for helium confined by a constant potential $\left(U_{0}=0.0 \mathrm{au}\right)$ at $R_{c}=1.0$ au (top) and $R_{c}=0.5$ au (bottom). For $\alpha=0.00$ and $\alpha=0.25$. PBE: solid line. PBE0: dotted-dashed line. HF: dashed line.

\subsection{Atoms confined by impenetrable walls}

In this section, the helium atom is confined within impenetrable walls, and we compare the results with those obtained for penetrable walls already discussed above. For a confinement imposed by impenetrable walls, the exchange energy obtained for one-parameter hybrid functionals with $\alpha=0.0$, $0.1, \ldots, 1.0$ is presented in figure 11 , where the behavior exhibited by this property is entirely different than that associated with the finite potentials (compare figures 2 and 11). We observe that hybrid exchange functionals decrease the exchange energy when impenetrable walls confine the atom, in the same way than HF method. Garza et al also observed this behavior by using a free basis-set code with LDA and B88 exchange-only functionals. [23] Thus, the confinement imposed by an infinite potential always decreases the exchange energy.

Although one-parameter hybrid exchange functionals preserve the exchange energy behavior, the difference between their results and the HF exchange is increased for small confinement radii as we may confirm with figure 12 . For small confinement radii HF represents the lower bound

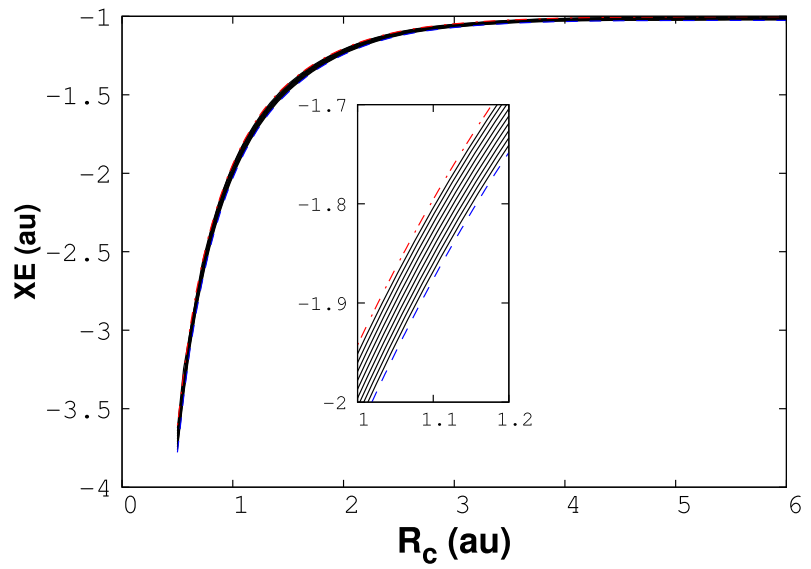

Figure 11. Exchange energy for helium atom confined by an infinite potential obtained by hybrid functionals with $\alpha=0.0,0.1, \ldots, 1.0$.

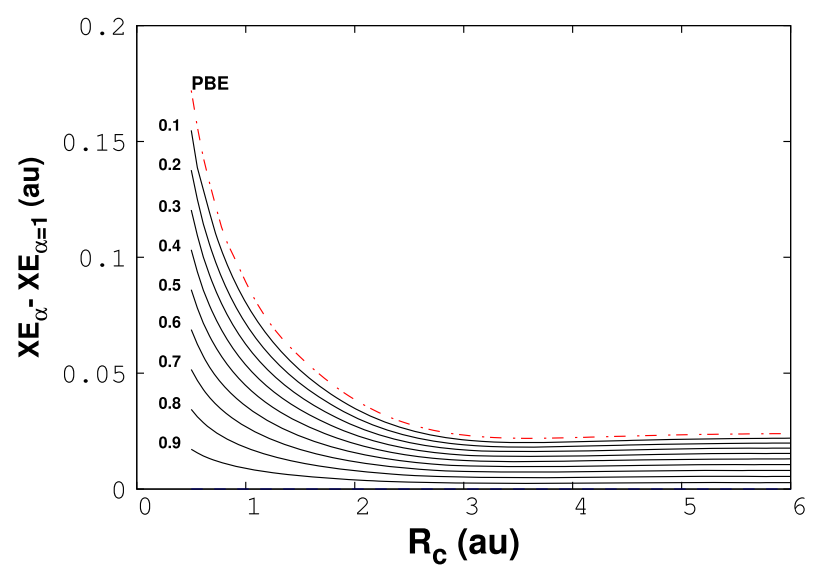

Figure 12. Exchange energy difference, $\mathrm{XE}_{\alpha}-\mathrm{XE}_{\alpha=1}$ for helium atom confined by an infinite potential obtained by hybrid functionals with $\alpha=0.0,0.1, \ldots, 1.0$.

and PBE the upper bound for the one-parameter hybrid exchange functionals considered in this article.

Many reports coincide with the fact that orbital energies are increased, for an infinite potential, when the confinement radius is reduced. For the one-parameter hybrid exchange functionals defined in equation (2), we also observe this behavior in figure 13. It is worth to note that for this confinement, orbital energies do not have an upper bound since we impose an infinite potential.

The difference between $\epsilon_{H}^{\alpha \neq 1}$ and $\epsilon_{H}^{\alpha=1}$ is also clear from figure 13 where always $\epsilon_{H}^{\alpha \neq 1}>\epsilon_{H}^{\alpha=1}$, without exception. However, it is evident that the behavior exhibited by $\epsilon_{H}^{\alpha \neq 1}$ is the same than that observed with $\operatorname{HF}(\alpha=1)$ for an arbitrary $\alpha$. Such a conclusion is not valid when the helium atom is confined by a finite potential (compare figures 6 and 13) for small confinement radii.

From these results, we conclude that for confinements where an infinite potential traps electrons, exchange functionals capture the main features of the exchange energy, when their results are contrasted with HF results. However, 


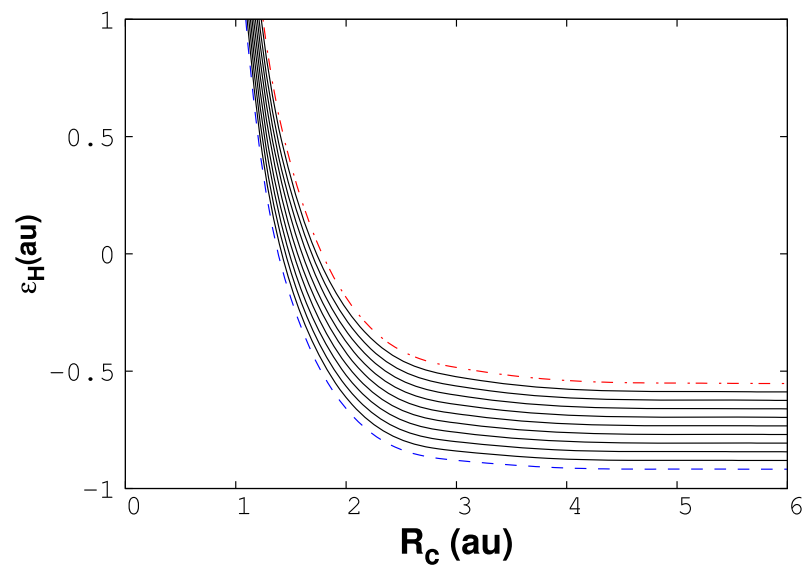

Figure 13. Highest occupied atomic orbital, $\epsilon_{H}$, for helium atom confined by a constant potential $U_{0}=0.0$ au obtained by hybrid functional with $\alpha=0.0,0.1, \ldots, 1.0$. $\mathrm{HF}(\alpha=1)$ results indicated with dashed line, $\operatorname{PBE}(\alpha=0)$ with dotted-dashed line and intermediate values in solid lines.

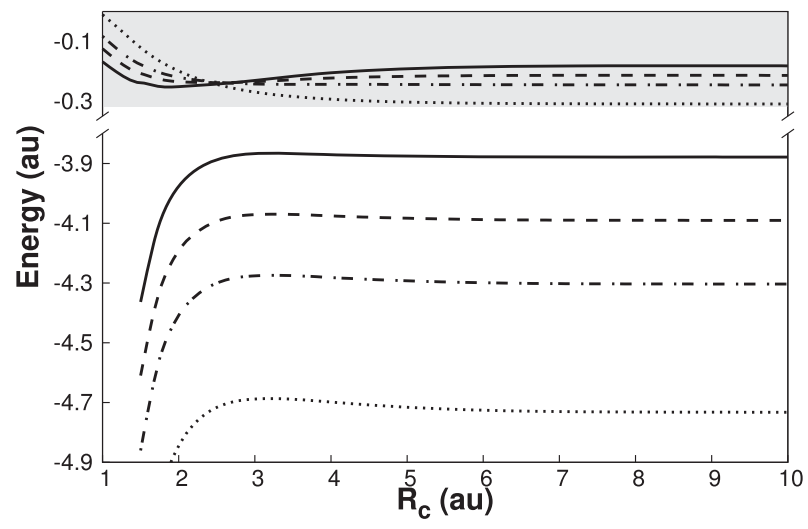

Figure 14. Orbital energies, 1s (blank zone) and 2s (gray zone), for beryllium confined by a constant potential with $U_{0}=0.0$ au and obtained by exchange hybrid potentials with $\alpha=0.0$ (solid line), $\alpha=0.25$ (dashed line), $\alpha=0.5$ (dotted-dashed line) and $\alpha=1.0$ (dotted line).

for confinements imposed by finite potentials, there are some cases where KS exchange functionals fail.

\subsection{Many-electron atoms}

The characteristics showed by the confined helium atom are also present in many-electron atoms, with their nuances. For example, the $2 \mathrm{~s}$ orbital of beryllium confined by a constant potential with $U_{0}=0.0$ au exhibits a similar behavior than that delivered by $\epsilon_{H}$ for the helium atom confined by the same confinement, and this is corroborated in figures 4, 6 and 14 . Thus, PBE predicts $\epsilon_{H}$ lower than HF for small confinement radii. Contributions of the $\mathrm{EE}$ in one-parameter hybrid exchange potentials partially alleviate some problems presented with PBE. By the side of the 1s orbital, all exchange functionals give the same behavior since all of them predict a lower orbital energy than that observed for the free atom for small confinement radii. This behavior was explained by
Rodriguez-Bautista et al regarding the delocalization of the electron density, which has an important impact on the effective nuclear charge exhibited by the confined atom [36]. It is worth to mention that HF predicts the lowest $1 \mathrm{~s}$ orbital energy and there is no crossing among the energies predicted by the hybrid exchange potentials used in this article. This result shows that confinement modifies no just the valence states since core states also change for an atom exposed to these spatial restrictions. All methods used to design effective core potentials assume that a physical or chemical process does not alter core states. Here we have shown that this assumption is not correct if an atom is under extreme confinements.

In table 4 we present the exchange energy for the beryllium atom confined by two constant potentials: $U_{0}=0.0 \mathrm{au}$ and $U_{0}=0.5 \mathrm{au}$. For our reference, $\alpha=1.0$, this atom shows important differences between $R_{c}=10.0$ and $R_{c}=1.5 \mathrm{au}$ for both barrier heights, since the exchange energy is reduced when a small $R_{c}$ confines the atom, and for both confinements, this quantity exhibits a minimum. One-parameter exchange functionals recover this behavior which is similar to that of the $\mathrm{He}$ atom in figure 2.

The prediction of PBE for the exchange energy is mapped directly on the TE, as we can see from table 5. Thus, for small confinement radii, the TE obtained by PBE is lower than that obtained by $\mathrm{HF}$ and the one-parameter hybrid exchange functionals do not make it right. This result is preserved for $\mathrm{Ne}, \mathrm{Mg}$ and $\mathrm{Ar}$ atoms, where PBE overestimates both the exchange and total energies for small confinement radii (see ESI). Thus, we conclude that PBE fails to describe correctly atoms confined by a constant potential and some of its deficiencies are partially corrected by oneparameter hybrid exchange functionals defined in equation (2).

\section{Conclusions}

One-parameter hybrid exchange functionals built as $E_{x}^{\mathrm{Hyb}}=E_{x}^{\mathrm{PBE}}+\alpha\left(\mathrm{EE}-E_{x}^{\mathrm{PBE}}\right)$ were tested over atoms confined by a constant potential. The results exhibited by PBE $(\alpha=0)$ coincide with those results predicted by the B88 exchange functional. Both exchange functionals overestimate the exchange energy for small confinement radii, and consequently, both GGA exchange functionals predict lower total energies than those delivered by HF. Orbital energies also present such an overestimation when we apply PBE or B88 on atoms confined by a constant potential with small $R_{c}$. Without a doubt LDA and GGA potentials give orbital energies higher than the orbital energies predicted by the HF potential. However, for $\mathrm{He}, \mathrm{Be}, \mathrm{Ne}$ and $\mathrm{Mg}$ the highest occupied atomic orbital is lower than that obtained by HF for small confinement radii. These results are different when the confinement is imposed by an infinite potential, and for this case PBE and hybrid exchange functionals predict exchange and orbital energies with the same trends exhibited by HF. The inclusion of a correction to the self-interaction error is an additional step to improve LDA and GGA functionals. 
Table 4. Exchange energy for beryllium confined by a constant potential with $U_{0}=0.0$ and $U_{0}=0.5$ au for several confinement radii, $R_{c}$. All quantities are reported in atomic units.

\begin{tabular}{lccccc}
\hline$R_{c}$ & 0.5 & 0.70 & 1.00 \\
\cline { 2 - 6 } & 0.00 & 0.25 & 0.50 & \\
\hline \multicolumn{5}{c}{$U_{0}=0.0 \mathrm{au}}$. \\
\hline 1.50 & -2.44437 & -2.41576 & -2.38965 & -2.37094 & -2.34756 \\
1.75 & -2.56001 & -2.53555 & -2.51181 & -2.49333 & -2.46644 \\
\hline 2.00 & -2.61241 & -2.59467 & -2.57764 & -2.56448 & -2.54548 \\
2.50 & -2.64401 & -2.63864 & -2.63456 & -2.63221 & -2.63019 \\
\hline 3.00 & -2.64808 & -2.64993 & -2.65318 & -2.65674 & -2.66364 \\
6.00 & -2.62935 & -2.63859 & -2.64859 & -2.65708 & -2.67061 \\
\hline 8.00 & -2.62784 & -2.63665 & -2.64622 & -2.65438 & -2.66744 \\
10.0 & -2.62743 & -2.63624 & -2.64579 & -2.65394 & -2.66697 \\
\hline & & & $U_{0}=0.5 \mathrm{au}$. & & \\
\hline 1.50 & -2.54391 & -2.51329 & -2.48402 & -2.46167 & -2.43022 \\
1.75 & -2.65205 & -2.62935 & -2.60737 & -2.59025 & -2.56529 \\
\hline 2.00 & -2.69548 & -2.68104 & -2.66755 & -2.65742 & -2.64330 \\
2.50 & -2.71117 & -2.70920 & -2.70843 & -2.70867 & -2.71039 \\
\hline 3.00 & -2.71439 & -2.71769 & -2.72181 & -2.72563 & -2.73218 \\
6.00 & -2.63669 & -2.64550 & -2.65507 & -2.66324 & -2.67630 \\
\hline 8.00 & -2.62896 & -2.63771 & -2.64721 & -2.65533 & -2.66831 \\
10.0 & -2.62765 & -2.63642 & -2.64594 & -2.65407 & -2.66707 \\
\hline
\end{tabular}

Table 5. Total energy for beryllium confined by a constant potential with $U_{0}=0.0$ au for several confinement radii, $R_{c}$. All quantities are reported in atomic units.

\begin{tabular}{lccccc}
\hline$R_{c}$ & \multicolumn{5}{c}{$\alpha$} \\
\cline { 2 - 6 } & 0.00 & 0.25 & 0.50 & 0.70 & 1.00 \\
\hline \multicolumn{5}{c}{$U_{0}=0.0 \mathrm{au}}$. \\
\hline 1.50 & -13.69202 & -13.67690 & -13.66379 & -13.65489 & -13.64448 \\
1.75 & -13.92823 & -13.90733 & -13.88713 & -13.87149 & -13.84892 \\
\hline 2.00 & -14.11933 & -14.09984 & -14.08079 & -14.06584 & -14.04390 \\
2.50 & -14.34317 & -14.33145 & -14.32028 & -14.31174 & -14.29958 \\
\hline 3.00 & -14.44887 & -14.44337 & -14.43853 & -14.43513 & -14.43080 \\
6.00 & -14.54379 & -14.54961 & -14.55593 & -14.56133 & -14.56995 \\
\hline 8.00 & -14.54490 & -14.55127 & -14.55805 & -14.56377 & -14.57281 \\
10.0 & -14.54500 & -14.55140 & -14.55821 & -14.56394 & -14.57301 \\
\hline & & & $U_{0}=0.5 \mathrm{au}$. & & \\
\hline 1.50 & -13.32704 & -13.30520 & -13.28461 & -13.26909 & -13.24752 \\
1.75 & -13.65620 & -13.63324 & -13.61077 & -13.59313 & -13.56723 \\
\hline 2.00 & -13.91093 & -13.89194 & -13.87338 & -13.85885 & -13.83755 \\
2.50 & -14.21599 & -14.20694 & -14.19847 & -14.19211 & -14.18325 \\
\hline 3.00 & -14.36288 & -14.36170 & -14.36091 & -14.36056 & -14.36047 \\
6.00 & -14.53959 & -14.54588 & -14.55258 & -14.55824 & -14.56719 \\
\hline 8.00 & -14.54445 & -14.55087 & -14.55771 & -14.56346 & -14.57254 \\
10.0 & -14.54494 & -14.55135 & -14.55817 & -14.56392 & -14.57299 \\
\hline
\end{tabular}




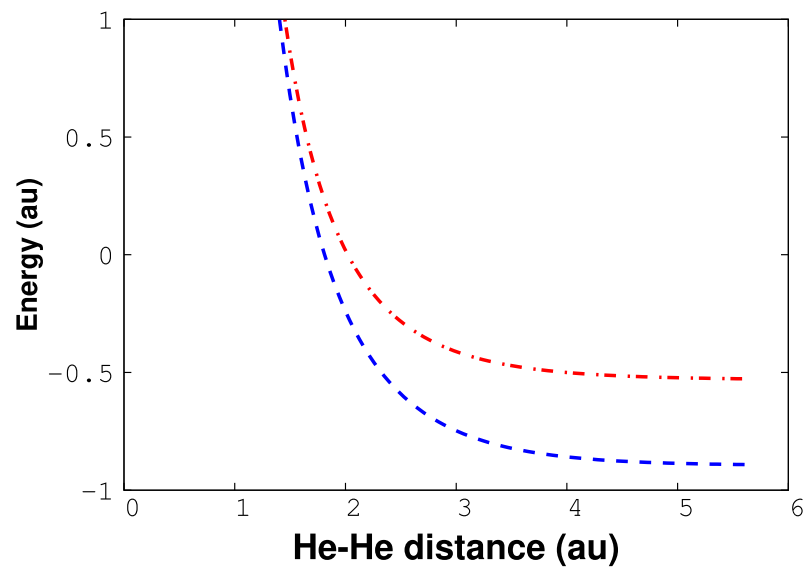

Figure 15. Top of the valence band for helium in an hexagon closepacked crystal structure obtained by Hartree-Fock (dashed line) and PBE (dotted-dashed line) methods.

Currently, in our group, we are working on this line to cancel this spurious contribution to study the performance of this approach on atoms confined by a constant potential.

We want to finish this article by giving one comment to the model of the confined atom. Gorecki and Byers-Brown defined one value of $U_{0}$ to match their results with experimental data associated with the kinetic energy for the confined helium atom. [30] In our case, we use the CRYSTAL code [52] to enclose a helium atom in a hexagon close-packed (hcp) crystal structure where the cell parameter was moved from 1.6 to $6.0 \AA$ by using 84 k-points in the irreducible Brillouin zone, and the basis set designed by Shäfer et al [53] In figure 15 we present one point associated to the top of the dispersion relationship $\epsilon_{\mathbf{k}}$ versus $\mathbf{k}$ as a function of the shortest $\mathrm{He}-\mathrm{He}$ distance in the hcp crystal structure for HF (dashed line) and PBE (dotted-dashed line) methods. From this plot, we show that the model of the confined atom can simulate the helium atom within a periodic system by using a considerable barrier height (compare figure 13 with figure 15). In this way, both the model of the confined atom and CRYSTAL show deficiencies of PBE compared with HF results. The observations mentioned in this article give some clues to several conclusions obtained for systems submitted under high pressures where GGAs functionals fail to correctly describe systems under these extreme conditions [54, 55].

In our opinion, the model of the confined atom is an useful tool to test exchange-correlation functionals. At this moment we are adapting other functionals like the SCAN exchange-correlation functional in the MEXICA-C code to study its performance in atoms confined by finite potentials [56] and methods based on the wave-function to estimate the correlation energy.

\section{Acknowledgments}

The authors thank the facilities provided by the Laboratorio de Supercómputo y Visualización en Paralelo at the Universidad Autónoma Metropolitana-Iztapalapa. F-A Duarte-Alcaráz, M-A
Martínez-Sánchez and M Rivera-Almazo thank CONACYT (México) for the scholarships 400393, 574390 and 633579, respectively. Partial financial support was provided by $\mathrm{CON}$ ACYT through the project FC-2016/2412.

\section{ORCID iDs}

Francisco-Adrián Duarte-Alcaráz (iD https://orcid.org/00000001-8973-6468

Michael-Adán Martínez-Sánchez (ib https://orcid.org/00000002-9661-8440

Marcos Rivera-Almazo (ib https://orcid.org/0000-00015404-9318

Rubicelia Vargas (1D https://orcid.org/0000-0003-0180-5727

Rodrigo-Arturo Rosas-Burgos (i) https://orcid.org/0000-

0003-1597-3326

Jorge Garza (iD https://orcid.org/0000-0003-4249-6078

\section{References}

[1] Michels A, De Boer J and Bijl A 1937 Physica 4 981-94

[2] Sommerfeld A and Welker H 1938 Ann. Phys. 32 56-65

[3] de Groot S R and ten Seldam C A 1946 Physica 12 669-82

[4] Ludeña E V 1978 J. Chem. Phys. 69 1770-5

[5] Connerade J P and Dolmatov V 1998 J. Phys. B: At. Mol. Opt. Phys. 31 3557-64

[6] Drake R P 2006 High-Energy-Density Physics: Fundamentals, Inertial Fusion, and Experimental Astrophysics (Berlin: Springer)

[7] Griem H R 1997 Principles of Plasma Spectroscopy (Cambridge Monographs on Plasma Physics) (Cambridge: Cambridge University Press)

[8] Graziani F, Desjarlais M, Redmer R and Trickey S (ed) 2014 Frontiers and Challenges in Warm Dense Matter (Lecture Notes in Computational Science and Engineering vol 96) (Cham: Springer)

[9] Ecker G and Kröll W 1963 Phys. Fluids 6 62-9

[10] Stewart J C and Pyatt K D Jr 1966 Astrophys. J. 144 1203-11

[11] More R M 1982 J. Quant. Spectrosc. Radiat. Transf. 27 345-57

[12] Salzmann D 1998 Atomic Physics in Hot Plasmas (International Series of Monographs) (Oxford: Oxford University Press)

[13] Li X, Rosmej F B, Lisitsa V S and Astapenko V A 2019 Phys. Plasmas 26033301

[14] Rosmej F B 2018 J. Phys. B: At. Mol. Opt. Phys. 51 09LT01

[15] Piron R and Blenski T 2011 Phys. Rev. E 83026403

[16] Connerade J P, Dolmatov V and Lakshmi P A 2000 J. Phys. B: At. Mol. Opt. Phys. 33 251-64

[17] Banerjee A, Kamal C and Chowdhury A 2006 Phys. Lett. A 350 121-5

[18] Sarsa A and Le Sech C 2011 J. Chem. Theory Comput. 7 2786-94

[19] Garza J, Hernández-Pérez J M, Ramírez J Z and Vargas R 2012 J. Phys. B: At. Mol. Opt. Phys. 45015002

[20] Sarsa A, Buendia E and Galvez F J 2016 J. Phys. B: At. Mol. Opt. Phys. 49145003

[21] Young T D, Vargas R and Garza J 2016 Phys. Lett. A 380 $712-7$

[22] Gálvez F J, Buendía E and Sarsa A 2017 Int. J. Quantum Chem. 117 e25421

[23] Garza J, Vargas R and Vela A 1998 Phys. Rev. E 58 3949-54

[24] Cruz S A 2009 Adv. Quantum Chem. 57 255-83 
[25] Garza J and Vargas R 2009 Adv. Quantum Chem. 57 241-54

[26] Díaz-García C and Cruz S A 2008 Int. J. Quantum Chem. 108 1572-88

[27] Waugh S, Chowdhury A and Banerjee A $2010 \mathrm{~J}$. Phys. B: At. Mol. Opt. Phys. 43225002

[28] Martínez-Sánchez M A, Bautista M R, Vargas R and Garza J 2016 Theor. Chem. Acc. 135207

[29] Ley-Koo E and Rubinstein S 1979 J. Chem. Phys. 71 351-7

[30] Gorecki J and Byers-Brown W 1988 J. Phys. B: At. Mol. Opt. Phys. 21 403-10

[31] Marin J L and Cruz S A 1992 J. Phys. B: At. Mol. Opt. Phys. 254365

[32] Jortner J and Coulson C A 1961 Mol. Phys. 24 451-64

[33] Martínez-Sánchez M A, Aquino N, Vargas R and Garza J 2017 Chem. Phys. Lett. 690 14-9

[34] Mccreery J H, Christoffersen R E and Hall G G 1976 J. Am. Chem. Soc. 98 7191-7

[35] Sako T, Paldus J and Diercksen G H F 2010 Phys. Rev. A 81 022501

[36] Rodriguez-Bautista M, Díaz-García C, Navarrete-López A M, Vargas R and Garza J 2015 J. Chem. Phys. 14334103

[37] Dirac P A M 1930 Proc. Camb. Phil. Soc. 26 376-85

[38] Becke A D 1988 Phys. Rev. A 38 3098-100

[39] Rodriguez-Bautista M, Vargas R, Aquino N and Garza J 2018 Int. J. Quantum Chem. 118 e25571

[40] Mardirossian N and Head-Gordon M 2017 Mol. Phys. 115 2315-72

[41] Sun J et al 2016 Nat. Chem. 8 831-6
[42] Perdew J P, Burke K and Ernzerhof M 1996 Phys. Rev. Lett. 77 3865-8

[43] Adamo C and Barone V 1999 J. Chem. Phys. 110 6158-70

[44] Guido C A, Bremond E, Adamo C and Cortona P 2013 J. Chem. Phys. 138021104

[45] Carmona-Espíndola J, Gázquez J L, Vela A and Trickey S B 2016 Theor. Chem. Acc. 135120

[46] Kohn W and Sham L J 1965 Phys. Rev. 140 A1133-8

[47] Szabo A and Ostlund N S 1996 Modern Quantum Chemistry: Introduction to Advanced Electronic Structure Theory (New York: Dover)

[48] García-Hernández E, Díaz-García C, Vargas R and Garza J 2014 J. Phys. B: At. Mol. Opt. Phys. 47185007

[49] Tatewaki H, Koga T, Sakai Y and Thakkar A J 1994 J. Chem. Phys. 101 4945-8

[50] Valiev M et al 2010 Comput. Phys. Commun. 181 1477-89

[51] Woon D E and Dunning T H 1994 J. Chem. Phys. 100 2975-88

[52] Dovesi R et al 2014 Int. J. Quantum Chem. 114 1287-317

[53] Schäfer A, Horn H and Ahlrichs R 1992 J. Chem. Phys. 972571

[54] Ochoa-Calle A J, Zicovich-Wilson C M, Hernández-Lamoneda R and Ramírez-Solís A 2015 J. Chem. Theory Comput. 11 1195-205

[55] Ochoa-Calle A J, Zicovich-Wilson C M and Ramírez-Solís A 2015 Phys. Rev. B 92085148

[56] Sun J, Ruzsinszky A and Perdew J P 2015 Phys. Rev. Lett. 115 036402 


\section{Bibliografía}

(1) Goodenough, J. Perspective on Engineering Transition-Metal Oxides. Chemistry of materials 2014, 26 (1), 820-829, DOI: 10.1021/cm402063u.

(2) Kung, H., Transition Metal Oxides: Surface Chemistry and Catalysis; Studies in Surface Science and Catalysis; Elsevier Science: 1989.

(3) Lany, S. Semiconducting transition metal oxides. Journal of Physics: Condensed Matter 2015, 27 (28), 283203, DOI: 10 . 1088 / 0953 - 8984 / 27 / 28 / 283203.

(4) Sinhamahapatra, A.; Jeon, J.-P.; Kang, J.; Han, B. y Yu, J.-S. Oxygen-Deficient Zirconia (ZrO2-x): A New Material for Solar Light Absorption. Scientific Reports 2016, 6 (1), 27218, DOI: 10 . 1038/srep27218.

(5) Tokura, Y. Correlated-electron physics in transition-metal oxides. Physics Today 2003, 56 (7), 50-55, DOI: 10.1063/1.1603080.

(6) Mattheiss, L. Electronic structure of rhombohedral $\mathrm{Ti}_{2} \mathrm{O}_{3}$. J. Phys.:Condens. Matter 1996, 8 (33), 5987-5995, DOI: 10 . 1088/0953-8984/8/33/007.

(7) Marques, M. A. L.; Vidal, J.; Oliveira, M. J. T.; Reining, L. y Botti, S. Densitybased mixing parameter for hybrid functionals. Phys. Rev. B 2011, 83 (3), 035119, DOI: 10.1103/PhysRevB.83.035119.

(8) Gerosa, M.; Bottani, C.; Caramella, L.; Onida, G.; di Valentin, C. y Pacchioni, G. Electronic structure and phase stability of oxide semiconductors: Performance of dielectric-dependent hybrid functional DFT, benchmarked against 
GW band structure calculations and experiments. Phys. Rev. B 2015, 91 (15), 155201, DOI: 10.1103/PhysRevB.91.155201.

(9) Singh, H.; Singh, M.; Kumar, S. y Kashyap, M. K. Full potential calculation of electronic properties of rutile $\mathrm{RO} 2(\mathrm{R}=\mathrm{Si}, \mathrm{Ge}, \mathrm{Sn}$ and $\mathrm{Pb})$ compounds via modified Becke Johnson potential. Physica B: Condensed Matter 2011, 406 (20), 3825-3830, DOI: 10 .1016/ j . physb .2011 .07.004.

(10) Sevik, C. y Bulutay, C. Theoretical study of the insulating oxides and nitrides: $\mathrm{SiO} 2, \mathrm{GeO} 2, \mathrm{Al} 2 \mathrm{O} 3, \mathrm{Si} 3 \mathrm{~N} 4$, and Ge3N4. Journal of Materials Science 2007, 42 (16), 6555-6565, DOI: 10 . 1007/s 10853-007-1526-9.

(11) Unal, H.; Gunceler, D.; Gulseren, O.; Ellialtioglu, S. y Mete, E. Hybrid functional calculated optical and electronic structures of thin anatase $\mathrm{TiO}_{2}$ nanowires with organic dye adsorbates. Appl. Surf. Sci. 2015, 354, 437-442, DOI: 10.1016/ J . APSUSC . 2015.04.086.

(12) Seo, D.; Urban, A. y Ceder, G. Calibrating transition-metal energy levels and oxygen bands in first-principles calculations: Accurate prediction of redox potentials and charge transfer in lithium transition-metal oxides. Phys. Rev. B 2015, 92 (11), 115118, DOI: 10.1103/PhysRevB.92.115118.

(13) Demichelis, R.; Civalleri, B.; Ferrabone, M. y Dovesi, R. On the performance of eleven DFT functionals in the description of the vibrational properties of aluminosilicates. Int. J. Quantum Chem. 2010, 110 (2), 406-415, DOI: 10 . 1002 / qua . 22301.

(14) De La Pierre, M.; Orlando, R.; Maschio, L.; Doll, K.; Ugliengo, P. y Dovesi, R. Performance of six functionals (LDA, PBE, PBESOL, B3LYP, PBE0, and WC1LYP) in the simulation of vibrational and dielectric properties of crystalline compounds. The case of forsterite $\mathrm{Mg}_{2} \mathrm{SiO}_{4}$. J. Comput. Chem. 2011, 32 (9), 1775-1784, DOI: 10.1002 / jcc. 21750.

(15) Többens, D. y Kahlenberg, V. Improved DFT calculation of Raman spectra of silicates. Vib. Spectrosc. 2011, 56 (2), 265-272, DOI: 10.1016/ J.VIBSPEC. 2011.04 .002$. 
(16) Burke, K.; Ernzerhof, M. y Perdew, J. P. The adiabatic connection method: a non-empirical hybrid. Chemical Physics Letters 1997, 265 (1-2), 115-120, DOI: 10.1016/S0009-2614(96)01373-5.

(17) Guido, C. A.; Brémond, E.; Adamo, C. y Cortona, P. Communication: One third: A new recipe for the PBE0 paradigm. The Journal of Chemical Physics 2013, 138 (2), 21104, DOI: 10.1063/1.4775591.

(18) Cortona, P. Note: Theoretical mixing coefficients for hybrid functionals. The Journal of Chemical Physics 2012, 136 (8), 86101, DOI: 10.1063/1.3690462.

(19) Koller, D.; Blaha, P. y Tran, F. Hybrid functionals for solids with an optimized Hartree-Fock mixing parameter. Journal of physics. Condensed matter : an Institute of Physics journal 2013, 25 (43), 435503, DOI: 10 . 1088/0953$8984 / 25 / 43 / 435503$.

(20) Szabo, A. y Ostlund, N. S., Modern Quantum Chemistry: Introduction to Advanced Electronic Structure Theory; Dover Publications Inc.: 1996.

(21) Peng, C.; Calvin, J. A. y Valeev, E. F. Coupled-cluster singles, doubles and perturbative triples with density fitting approximation for massively parallel heterogeneous platforms. International Journal of Quantum Chemistry 2019, 119 (12), e25894, DOI: 10 . 1002/qua . 25894.

(22) Koch, W. y Holthausen, M. C., A Chemist's Guide to Density Functional Theory; Wiley - VCH: 2da ed., 2001.

(23) Engel, E. y Dreizler, R., Density Functional Theory: An Advanced Course; Theoretical and Mathematical Physics; Springer Berlin Heidelberg: 2011.

(24) Perdew, J. P. y Schmidt, K. Jacob's ladder of density functional approximations for the exchange-correlation energy. AIP Conference Proceedings 2001, 577 (1), 1-20, DOI: 10.1063/1.1390175.

(25) Becke, A. D. A new mixing of Hartree-Fock and local density-functional theories. The Journal of Chemical Physics 1993, 98 (2), 1372-1377, DOI: 10 . $1063 / 1.464304$. 
(26) Perdew, J. P.; Ernzerhof, M. y Burke, K. Rationale for mixing exact exchange with density functional approximations. The Journal of Chemical Physics 1996, 105 (22), 9982-9985, DOI: 10.1063/1.472933.

(27) Adamo, C. y Barone, V. Toward reliable density functional methods without adjustable parameters: The PBE0 model. The Journal of Chemical Physics 1999, 110 (13), 6158-6170, DOI: 10 . 1063/1.478522.

(28) Shimazaki, T. y Asai, Y. Band structure calculations based on screened Fock exchange method. Chemical Physics Letters 2008, 466 (1-3), 91-94, DOI: 10. 1016 / J . CPLETT . 2008 . 10.012.

(29) Viñes, F.; Lamiel-García, O.; Chul Ko, K.; Yong Lee, J. e Illas, F. Systematic study of the effect of HSE functional internal parameters on the electronic structure and band gap of a representative set of metal oxides. Journal of Computational Chemistry 2017, 38 (11), 781-789, DOI: 10.1002/ jcc . 24744.

(30) H Skone, J.; Govoni, M. y Galli, G. Self-consistent hybrid functional for condensed systems. Physical Review B 2015, 89, DOI: 10 . 1103/PhysRevB . 89 . 195112.

(31) Viñes, F.; Lamiel-García, O.; Chul Ko, K.; Yong Lee, J. e Illas, F. Systematic study of the effect of HSE functional internal parameters on the electronic structure and band gap of a representative set of metal oxides. Journal of Computational Chemistry 2017, 38 (11), 781-789, DOI: 10.1002 / jcc . 24744.

(32) Miceli, G.; Chen, W.; Reshetnyak, I. y Pasquarello, A. Nonempirical hybrid functionals for band gaps and polaronic distortions in solids. Physical Review B 2018, 97, DOI: 10.1103/PhysRevB.97.121112.

(33) Dovesi, R.; Civalleri, B.; Orlando, R.; Roetti, C. y Saunders, V. R. en Reviews in Computational Chemistry, Lipkowitz, K. B., Larter, R. y Cundari, T. R., eds.; Wiley: 2005; vol. 21; cap. 1, págs. 1-125. 
(34) Dovesi, R.; Pisani, C.; Roetti, C.; Causà, M. y Saunders, V. R. CRYSTAL88, An ab initio all-electron LCAO-Hartree-Fock program for periodic systems. QCPE PGM N.577 1989.

(35) Dovesi, R.; Erba, A.; Orlando, R.; Zicovich-Wilson, C. M.; Civalleri, B.; Maschio, L.; Rérat, M.; Casassa, S.; Baima, J.; Salustro, S. y Kirtman, B. Quantummechanical condensed matter simulations with CRYSTAL. Wiley Interdisciplinary Reviews: Computational Molecular Science 2018, (December 2017), e1360, DOI: 10.1002/wcms . 1360.

(36) Demichelis, R. y Dovesi, R. Discussion about some parts of the CRYSTAL code - Work in progress. 2008, http: / / www. crystal . unito. it/documentation. pdf.

(37) Tinkham, M., Group Theory and Quantum Mechanics; Dover Books on Chemistry; Dover Publications: 2012.

(38) McMurchie, L. E. y Davidson, E. R. One- and two-electron integrals over cartesian gaussian functions. Journal of Computational Physics 1978, 26 (2), 218-231, DOI: 10 . 1016/0021-9991(78)90092-X.

(39) McArdle, S.; Endo, S.; Aspuru-Guzik, A.; Benjamin, S. y Yuan, X. Quantum computational chemistry. 2018, arXiv: http : / / arxiv . org / abs / 1808 . 10402.

(40) Zahran, M. Heterogeneous Computing: Here to Stay. Queue 2016, 14 (6), 40:31-40:42, DOI: 10 . 1145/3028687 . 3038873.

(41) Strohmaier, E.; Simon, H.; Dongarra, J. y Meuer, M. Lista Top500 de Noviembre 2018. 2018, https://www. top500.org/lists/2018/11/.

(42) Sanders, J. y Kandrot, E., CUDA by Example: An Introduction to GeneralPurpose GPU Programming; Pearson Education: 2010.

(43) Rahman, R., Intel Xeon Phi Coprocessor Architecture and Tools: The Guide for Application Developers, 1st; Apress: Berkely, CA, USA, 2013. 
(44) NVIDIA Corporation PGI Compilers \& Tools., ht tps : / / www . pgroup . com, Consultado el 20 de Mayo del 2019., 2019.

(45) Farber, R., Parallel Programming with OpenACC; Elsevier Science: 2016.

(46) Chandrasekaran, S. y Juckeland, G., OpenACC for Programmers: Concepts and Strategies; Pearson Education: 2017.

(47) Eriksen, J. J. Efficient and portable acceleration of quantum chemical manybody methods in mixed floating point precision using OpenACC compiler directives. Molecular Physics 2017, 115 (17-18), 2086-2101, DOI: 10 . 1080 / 00268976.2016 .1271155$.

(48) NVIDIA Corporation Quantum Chemistry GPU Benchmarking Report March 2018., https : / / www . nvidia . com / ob ject / computational_chemistry . html, Consultado el 20 de Mayo del 2019., 2018.

(49) Dovesi, R.; Orlando, R.; Erba, A.; Zicovich-Wilson, C. M.; Civalleri, B.; Casassa, S.; Maschio, L.; Ferrabone, M.; De La Pierre, M.; D’Arco, P.; Noël, Y.; Causà, M.; Rérat, M. y Kirtman, B. CRYSTAL14: A program for the ab initio investigation of crystalline solids. International Journal of Quantum Chemistry 2014, 114 (19), 1287-1317, DOI: 10. 1002/qua. 24658.

(50) Wolf, L. The GPU Revolution. Chemical E Engineering News 2010, 88 (44), 27-29.

(51) IBM Power Systems., https://www . ibm.com/mx-es/it-infrastructure/ power, Consultado el 20 de Mayo del 2019., 2019.

(52) OpenPOWER Fundation OpenPOWER Fundation., ht tps: / / openpower foundation. org, Consultado el 20 de Mayo del 2019., 2019.

(53) The Open MPI Project MPI-Init-thread(3) man page., https : / / www . openmpi .org/doc/current/man3/MPI_Init_thread.3.php, Consultado el 20 de Mayo del 2019., 2019. 
(54) Rabenseifner, R.; Hager, G. y Jost, G. Hybrid MPI and OpenMP Parallel Programming., https : / / openmp . org / wp - content / uploads / HybridPP_ Slides.pdf, Consultado el 20 de Mayo del 2019., 2013.

(55) OpenBLAS OpenBLAS: An optimized BLAS library., ht tps: / / www openblas . net/, Consultado el 20 de Mayo del 2019., 2019.

(56) IBM Engineering and Scientific Subroutine Library., https : / / www . ibm . com/support/knowledgecenter/en/SSFHY8/essl_welcome.html, Consultado el 20 de Mayo del 2019., 2016.

(57) Erba, A.; Baima, J.; Bush, I.; Orlando, R. y Dovesi, R. Large-Scale Condensed Matter DFT Simulations: Performance and Capabilities of the CRYSTAL Code. Journal of Chemical Theory and Computation 2017, 13 (10), 5019-5027, DOI: 10.1021/acs. jctc. 7b00687.

(58) Gillen, R. y Robertson, J. Accurate screened exchange band structures for the transition metal monoxides $\mathrm{MnO}, \mathrm{FeO}, \mathrm{CoO}$ and $\mathrm{NiO}$. Journal of physics. Condensed matter : an Institute of Physics journal 2013, 25 (16), 165502, DOI: 10 . 1088/0953-8984/25/16/165502.

(59) Kobayashi, S.; Nohara, Y.; Yamamoto, S. y Fujiwara, T. GW approximation with LSDA + $\mathrm{U}$ method and applications to $\mathrm{NiO}, \mathrm{MnO}$, and $\mathrm{V}_{2} \mathrm{O}_{3}$. Phys. Rev. B 2008, 78 (15), 155112, DOI: 10.1103/PhysRevB .78.155112.

(60) Gallino, F.; Di Valentin, C. y Pacchioni, G. Band gap engineering of bulk ZrO2 by Ti doping. Physical Chemistry Chemical Physics 2011, 13 (39), 17667, DOI: $10.1039 /$ c1cp21987a.

(61) Towler, M.; Allan, N.; Harrison, N.; Saunders, V.; Mackrodt, W. y Apra, E. Ab initio study of MnO and NiO. Phys. Rev. B 1994, 50 (8), 5041, DOI: 10 . 1103/PhysRevB.50.5041.

(62) CRYSTAL Theoretical Chemistry Group Basis sets library., http : / / www . crystal .unito.it/basis-sets . php, Consultado el 20 de Mayo del 2019., 2019. 
(63) Rivera-Almazo, M. Estudio teórico del MnO., Proyecto Terminal. Licenciatura en Química. Universidad Autónoma Metropolitana - Iztapalapa, 2016.

(64) Leger, J. M.; Tomaszewski, P. E.; Atouf, A. y Pereira, A. S. Pressure-induced structural phase transitions in zirconia under high pressure. Phys. Rev. B 1993, 47 (21), 14075-14083, DOI: 10.1103/PhysRevB .47.14075.

(65) Smith, C. F. y Crandall, W. B. Calculated High-Temperature Elastic Constants for Zero Porosity Monoclinic Zirconia. Journal of the American Ceramic Society 1964, 47 (12), 624-627, DOI: 10 .1111/ j .1151-2916 . 1964 . tb13120. $x$.

(66) Mashimo, T.; Nagayama, K. y Sawaoka, A. Shock compression of zirconia $\mathrm{ZrO} 2$ and zircon $\mathrm{ZrSiO} 4$ in the pressure range up to $150 \mathrm{GPa}$. Physics and Chemistry of Minerals 1983, 9 (6), 237-247, DOI: 10 . 1007/BF 00309573.

(67) Jaffe, J. E.; Bachorz, R. A. y Gutowski, M. Low-temperature polymorphs of $\mathrm{ZrO}_{2}$ and $\mathrm{HfO}_{2}$ : A density-functional theory study. Phys. Rev. B 2005, 72 (14), 144107, DOI: 10.1103/PhysRevB.72.144107.

(68) Kuwabara, A.; Tohei, T.; Yamamoto, T. y Tanaka, I. Ab initio lattice dynamics and phase transformations of $\mathrm{ZrO}_{2}$. Phys. Rev. B 2005, 71 (6), 064301, DOI: 10.1103/PhysRevB.71.064301.

(69) Cousland, G.; Cui, X.; Smith, A.; Stampfl, A. y Stampfl, C. Mechanical properties of zirconia, doped and undoped yttria-stabilized cubic zirconia from first-principles. Journal of Physics and Chemistry of Solids 2018, 122, 51-71, DOI: $10.1016 /$ j . jpcs.2018.06.003.

(70) Fujimoto, M.; Akahama, Y.; Fukui, H.; Hirao, N. y Ohishi, Y. Observation of the negative pressure derivative of the bulk modulus in monoclinic $\mathrm{ZrO} 2$. AIP Advances 2018, 8 (1), 015310, DOI: 10.1063/1.5017774.

(71) Leger, J. M.; Tomaszewski, P. E.; Atouf, A. y Pereira, A. S. Pressure-induced structural phase transitions in zirconia under high pressure. Phys. Rev. B 1993, 47 (21), 14075-14083, DOI: 10.1103/PhysRevB. 47 . 14075. 
(72) Desgreniers, S. y Lagarec, K. High-density $\mathrm{ZrO}_{2}$ and $\mathrm{HfO}_{2}$ : Crystalline structures and equations of state. Phys. Rev. B 1999, 59 (13), 8467-8472, DOI: 10.1103/PhysRevB.59.8467.

(73) Wyckoff, R., Crystal Structures; Wiley: New York, 1963.

(74) Howard, C. J.; Hill, R. J. y Reichert, B. E. Structures of $\mathrm{ZrO}_{2}$ polymorphs at room temperature by high-resolution neutron powder diffraction. Acta Crystallographica Section B 1988, 44 (2), 116-120, DOI: 10.1107/S0108768187010279.

(75) Yamanaka, T.; Kurashima, R. y Mimaki, J. X-ray diffraction study of bond character of rutile-type $\mathrm{SiO}_{2}, \mathrm{GeO}_{2}$ and $\mathrm{SnO}_{2}$. Z. Kristallogr. 2000, 215 (7), 424-428, DOI: 10.1524/zkri.2000.215.7.424.

(76) Ohnishi, S. y Mizutani, H. Crystal field effect on bulk moduli of transition metal oxides. J. Geophys. Res. Solid Earth 1978, 83 (B4), 1852-1856, DOI: 10. 1029 / JB083iB04p01852.

(77) Noguchi, Y.; Kusaba, K.; Fukuoka, K. y Syono, Y. Shock-induced phase transition of MnO around 90GPa. Geophys. Res. Lett. 1996, 23 (12), 1469-1472, DOI: 10.1029/96GL01326.

(78) Makino, Y. y Miyake, S. Estimation of bulk moduli of compounds by empirical relations between bulk modulus and interatomic distance. J. Alloys Compd. 2000, 313 (1-2), 235-241, DOI: 10.1016/S0925-8388 (00) 01182-8.

(79) Jeanloz, R. y Rudy, A. Static compression of MnO manganosite to $60 \mathrm{GPa}$. J. Geophys. Res. Solid Earth 1987, 92 (B11), 11433-11436, DOI: 10 . 1029 / JB092iB11p11433.

(80) Demuth, T.; Jeanvoine, Y.; Hafner, J. y Angyan, J. Polymorphism in silica studied in the local density and generalized-gradient approximations. J. Phys.:Condens. Matter 1999, 11 (19), 3833-3874, DOI: 10 . 1088 / 0953 $8984 / 11 / 19 / 306$. 
(81) Weidner, D.; JD, B.; Ringwood, A. y Sinclair, W. The single-crystal elasticmoduli of stishovite. J. Geophys. Res. 1982, 87 (B6), 4740-4746, DOI: 10 . 1029 / JB087iB06p04740.

(82) Panero, W.; Benedetti, L. y Jeanloz, R. Equation of state of stishovite and interpretation of $\mathrm{SiO}_{2}$ shock-compression data. J. Geophys. Res. Solid Earth 2003, 108 (B1), ECV5-1, DOI: 10.1029/2001JB001663.

(83) Christie, D. y Chelikowsky, J. Electronic and structural properties of germania polymorphs. Phys. Rev. B 2000, 62 (22), 14703-14711, DOI: 10 . 1103 / PhysRevB.62.14703.

(84) Wang, H. y G, S. Elasticity of some mantle crystal-structures: 2. Rutile $\mathrm{GeO}_{2}$. J. Geophys. Res. 1973, 78 (8), 1262-1273, DOI: 10.1029/ JB078i008p01262.

(85) Liebermann, R. Elastic properties of polycrystalline $\mathrm{SnO}_{2}$ and $\mathrm{GeO}_{2}$ : Comparison with stishovite and rutile data. Phys. Earth Planet. Inter. 1973, 7 (4), 461-465, DOI: 10.1016/0031-9201(73)90026-5.

(86) Haines, J. y Léger, J. X-ray diffraction study of the phase transitions and structural evolution of tin dioxide at high pressure: Relationships between structure types and implications for other rutile-type dioxides. Phys. Rev. B 1997, 55 (17), 11144-11154, DOI: 10.1103/PhysRevB.55.11144.

(87) Chang, E. y Graham, E. The elastic-constants of cassiterite $\mathrm{SnO}_{2}$ and their pressure and temperature-dependence. J. Geophys. Res. 1975, 80 (17), 2595-2599, DOI: 10.1029/ JB080i017p02595.

(88) Maj, S. Energy-gap and density in $\mathrm{SiO}_{2}$ polymorphs. Phys. Chem. Miner. 1988, 15 (3), 271-273, DOI: 10.1007/BF 00307516.

(89) Dash, L. K.; Vast, N.; Baranek, P.; Cheynet, M.-C. y Reining, L. Electronic structure and electron energy-loss spectroscopy of $\mathrm{ZrO}_{2}$ zirconia. Phys. Rev. B 2004, 70 (24), 245116, DOI: 10.1103/PhysRevB .70.245116. 
(90) French, R. H.; Glass, S. J.; Ohuchi, F. S.; Xu, Y.-N. y Ching, W. Y. Experimental and theoretical determination of the electronic structure and optical properties of three phases of $\mathrm{ZrO}_{2}$. Phys. Rev. B 1994, 49 (8), 5133-5142, DOI: 10.1103/PhysRevB.49.5133.

(91) Stapelbroek, M. y Evans, B. Exciton structure in UV-absorption edge of tetragonal $\mathrm{GeO}_{2}$. Solid State Commun. 1978, 25 (11), 959-962, DOI: 10 . 1016 / 0038-1098(78)90311-3.

(92) Kurmaev, E.; Wilks, R.; Moewes, A.; Finkelstein, L.; Shamin, S. y Kuneš, J. Oxygen $\mathrm{x}$-ray emission and absorption spectra as a probe of the electronic structure of strongly correlated oxides. Phys. Rev. B 2008, 77 (16), 165127, DOI: $10.1103 /$ PhysRevB.77.165127.

(93) Batzill, M. y Diebold, U. The surface and materials science of tin oxide. Prog. Surf. Sci. 2005, 79 (2-4), 47-154, DOI: 10.1016/ j .progsurf .2005.09.002.

(94) Alcaraz, F. A. D.; Martinez-Sanchez, M.-A.; Rivera-Almazo, M.; Vargas, R.; Rosas-Burgos, R. A. y Garza, J. Testing one-parameter hybrid exchange functionals in confined atomic systems. Journal of Physics B: Atomic, Molecular and Optical Physics 2019, DOI: 10 . 1088/1361-6455/ab233b.

(95) Navarrete-López, A. M.; Rivera-Almazo, M.; Garza, J. y Vargas, R. Importance of one-parameter hybrid exchange-correlation functionals in band gaps of transition metal and metalloid oxides. Theoretical Chemistry Accounts 2018, 137 (3), 36, DOI: 10 . 1007 / s00214-018-2222-3. 
Electo del intercambio

exacto sobre el Band Gap en

óxidos metálicos.
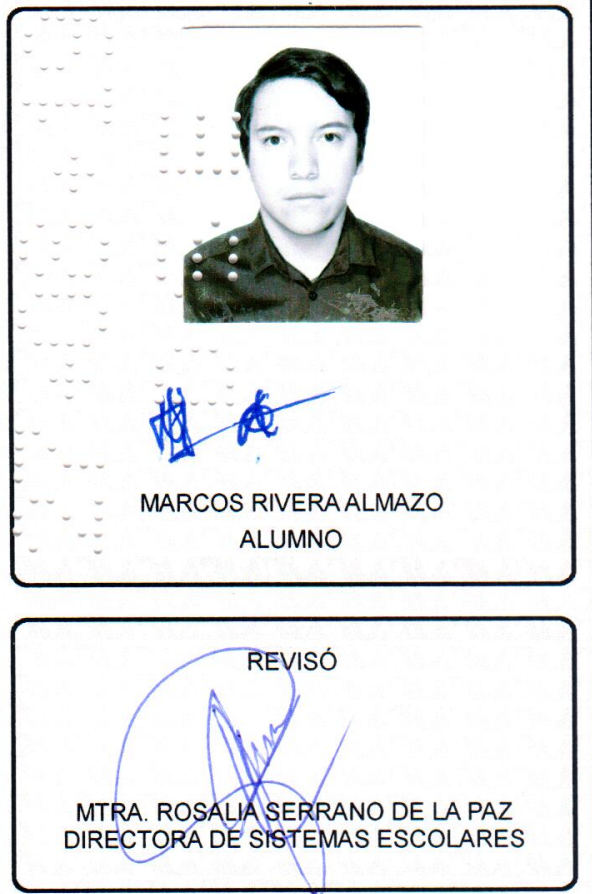

DIRECTOR DE LA DIVISIÓN DE CBI

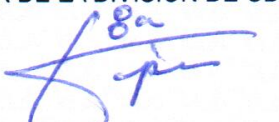

DR. JESUS ALBERTO OCHOA TAPIA

$y$ de acuerdo con el articulo 78 fracción III del Reglamento de Estudios Superiores de la Universidad Autónoma Metropolitana, los miembros del jurado resolvieron:

APROBAR

Acto continuo, el presidente del jurado comunicó al interesado el resultado de la evaluación y, en caso aprobatorio, le fue tomada la protesta.
En la Ciudad de México, se presentaron a las 10:00 horas del día 24 del mes de julio del año 2019 en la Unidad Iztapalapa de la Universidad Autónoma Metropolitana, los

DR. MARCELO ENRIOUE GALVAN ESPINOSA

DR. JORGE GARZA OLGUIN

Bajo la Presidencia del primero y con carácter de Secretario el último, se reunieron para proceder al Examen de Grado cuya denominación aparece al margen, para la obtención del grado de:

MAESTRO EN CIENCIAS (QUIMICA)

DE: MARCOS RIVERA ALMAZO
DR. JOSE EDUARDO BARRIOS VARGAS 


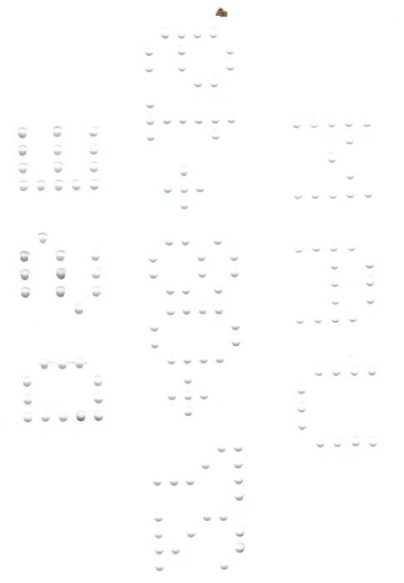

